\title{
Flutuações da Interface em Sistemas de Spins Bidimensionais
}

\author{
Elisabeti Kira
}

Dissertação apresentada

ao

Instituto de Matemática e Estatística

da

Universidade de São Paulo

para obtenção do grau de mestre

em

Estatística

Área de Concentração: Probabilidades

Orientador: Prof. Dr. Pablo Augusto Ferrari

-São Paulo, Novembro de 1989- 


\section{Agradecimentos}

No decorrer da elaboração deste trabalho ${ }^{1}$ contei com o apoio e incentivo de vários amigos e colegas. A eles meu muito obrigado!

Não posso, no entanto, deixar de considerar algumas pessoas que mais efetivamente contribuiram para a minha formação e para a realização deste estudo. Estou especialmente agradecida a:

- Pablo A. Ferrari pela sua paciente orientação e por ter me transmitido que a intuição matemática é tão importante quanto a sua formalização.

- E. Jordão Neves, João C. Barata, Nelson I. Tanaka, Pierre Picco, Rinaldo B. Schinazi e Roberto H. Schonmann pelas sugestões e proveitosas (pelo menos para mim) discussões.

- Antonio Carlos Pedroso de Lima por tudo.

Agradeço também à Capes e ao CNPq pela bolsa que me concederam nos primeiros anos do meu programa de mestrado.

\footnotetext{
${ }^{1} \mathrm{~A}$ edição deste trabalho foi preparada no sistema $\operatorname{LAT}_{\mathrm{E}} \mathrm{X}$
} 


\section{ÍNDICE}

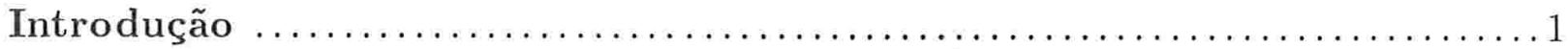

Capítulo I O Modelo de Interface S.O.S. ........................ 5

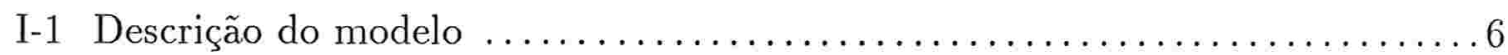

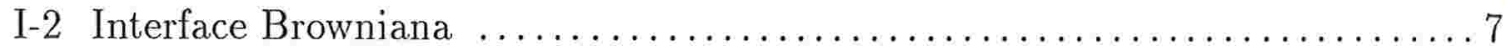

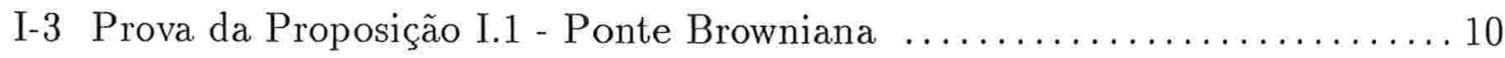

Capítulo II Interface no modelo de Ising bidimensional . . . . . . . . . . . . 17

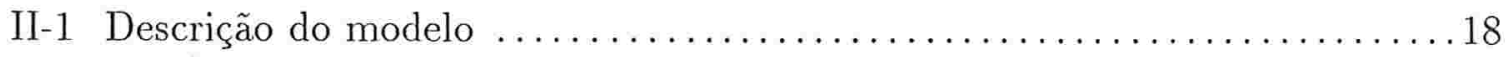

II-2 Expansão em Conglomerados de Contornos $\ldots \ldots \ldots \ldots \ldots \ldots \ldots \ldots \ldots \ldots \ldots \ldots \ldots \ldots$

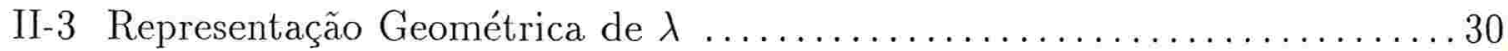

II-4 Potencial do Gás de Formas .................................... 32

II-5 Expansão em Conglomerados do Gás de Formas .................... 36

II-6 Teorema Limite Central para os saltos sobre intervalos .................42

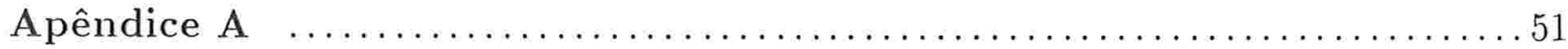

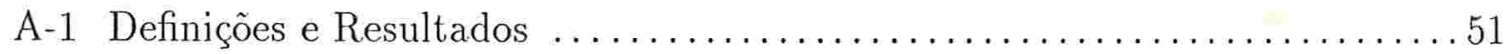

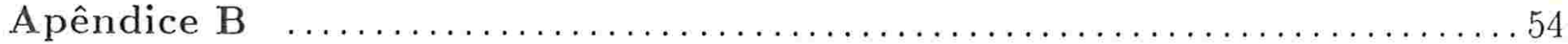

B-1 Expansão em Conglomerados de Contornos .......................55

B-2 Expansão em Conglomerados do Gás de Formas .................... 59

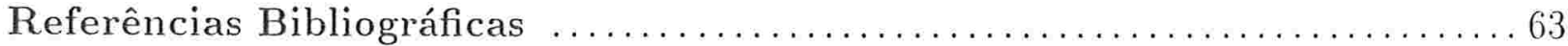




\section{Introdução}

O estudo de superfícies aleatórias é de interesse da mecânica estatística, que investiga e tenta relacionar propriedades macroscópicas de sistemas com muitos subsistemas microscópicos que interagem entre si.

Neste trabalho estudamos o comportamento macroscópico da superfície aleatória em sistemas nos quais a interação existe apenas entre os vizinhos mais próximos. Consideramos o modelo de Ising ferromagnético em duas dimensões, sem campo magnético externo e com condições de fronteira adequadas de modo a podermos definir uma linha aleatória, como veremos adiante. A definição deste modelo é simples: consideramos uma rede na. qual cada sítio tem um spin, com sinal positivo ou negativo, que vai interagir com seus dois vizinhos na direção horizontal e seus outros dois vizinhos na direção vertical. A interação é tal que favorece o alinhamento dos spins, isto é, dá maior peso a configurações em que os spins têm mesmo sinal, razão pela qual o modelo é denominado de ferromagnético.

Tomamos por rede a rede $\mathbb{Z}^{2}$ e restringimos originalmente o sistema a uma caixa. quadrada na qual colocamos spins positivos na metade superior da fronteira da caixa e spins negativos na metade inferior como condições de fronteira. A cada configuração de spins temos associada uma configuração de contornos (linhas conexas) na rede dual (a. rede transladada de $(1 / 2,1 / 2)$ da rede original), da seguinte maneira: a cada par de spins vizinhos com sinais opostos fazemos corresponder um segmento unitário na rede dual. Esses segmentos conexos formam contornos que separam spins com sinais opostos. A linha aleatória ou interface é justamente o contorno aberto que vaj do centro do lado esquerdo ao centro do lado direito da caixa, separando a região em que os spins são 
predominantemente positivos (parte superior da caixa) da região em que os spins são predominantemente negativos (parte inferior). O contornos fechados correspondem aos agrupamentos de spins de mesmo sinal que ocorrem em uma das duas regiões mencionadas. Por exemplo, na parte superior da caixa existem spins negativos que podem estar isolados ou agrupados. Denominaremos esses agrupamentos informalmente por ilhas.

Observamos que o comportamento desse sistema depende da temperatura a que está exposto. Se a temperatura for nula, o sistema fica congelado e a linha de separação é lisa (horizontal, reta) ao passo que se a temperatura for muito alta, o sistema fica caótico e, macroscopicamente, espera-se que a espessura (a altura máxima menos a mínima) da linha seja da ordem da largura da caixa e, nesse caso, no limite termodinâmico, a linha não seria bem definida. Veremos que para temperaturas suficientemente baixas, na escala da raiz quadrada da largura da caixa, a linha é bem definida. Um outro problema que encontramos ao estudar a interface, é que ela pode conter rugas, tornando a análise um pouco complexa. Entendemos por rugas trechos da linha, considerando-a dotada de uma orientação (sentido), nos quais a mesma retorna no sentido horizontal. Uma maneira de contornar o problema dessas ilhas e rugas é através de uma simplificação do sistema. Para tanto vamos introduzir um modelo denominado modelo de interface S.O.S..

A denominação S.O.S. vem de "Solid On Solid" porque o modelo em duas dimensões pode ser imaginado como o resultado de quadrados unitários que vão se sobrepondo, na direção vertical, de maneira aleatória para cada sítio na horizontal. Ao final deste procedimento temos uma linha aleatória que não contém rugas. Este novo modelo pode ser encarado como uma simplificação do anterior porque ele pode ser obtido a partir do Ising, tomando as interações na direção vertical com peso infinito e mantendo as da direção horizontal fixas. Olhando na direção vertical, abaixo do primeiro spin negativo todos os outros spins serão negativos; e acima todos serão positivos. Logo, teremos uma linha sem rugas separando os spins positivos dos negativos. Além disso, o sistema não contém ilhas de spins de sinais opostos. O que ocorre na realidade é que configurações com ilhas e rugas terão peso de Gibbs nulo.

Portanto, para o S.O.S., a parte relevante é a diferença da posição da interface em sítios vizinhos, de modo que se cada abscissa (sítio) tem a ordenada representando a altura da interface. A energia associada a esse modelo é a soma dos valores absolutos da diferença de altura de um sítio para outro. Essa diferença pode ser interpretada como o 
salto que a interface dá em cada sítio e, sem as rugas e as ilhas veremos que esses saltos têm mesma distribuição de probabilidades e são independentes. Assim sendo, a altura da interface num determinado sítio é a soma dos saltos até aquele sítio, ou seja, é a soma de variáveis aleatórias independentes e identicamente distribuídas.

Para obtermos o comportamento macroscópico, fazemos uma mudança de escala: a abscissa será o tempo na escala $N$ (largura da caixa) e a ordenada será a posição na escala $\sqrt{N}$. É sabido, pelo teorema de Donsker (ver, por exemplo, [Bil] ou o apêndice A deste trabalho), que um processo assim definido converge para um movimento Browniano unidimensional. Uma ressalva a ser feita é que no nosso modelo original, devido às condições de fronteira, temos ambas as bordas presas, i.e., as posições inicial e final são fixas e iguais a zero. A condição da posição inicial ser zero é usual mas a posição final ser fixa altera um pouco o resultado.

Billingsley provou em [Bil] que o movimento Browniano condicionado a que no instante $t=1$ ele esteja no zero (tomando cuidado com o fato desse evento ter probabilidade nula) é de fato uma ponte Browniana. Ora, então poderíamos imaginar que o processo que definimos, no qual estamos impondo a mesma condição (estar no zero no instante 1), converge para uma ponte Browniana. No primeiro capítulo esse resultado é apresentado e utilizado no caso particular do modelo de interface S.O.S., fornecendo, portanto, que o comportamento macroscópico da interface é o de uma ponte Browniana, tendo assim uma flutuaçấo da ordem de $\sqrt{N}$. A prova do resultado geral a respeito da ponte Browniana se encontra na seção I.3 e é feita em duas etapas: a primeira consiste em provar a. convergência da distribuição finito-dimensional e a segunda em provar que essa sequência de distribuiçôes preserva massa (é "tight"). Utilizamos para isso fortemente o fato que o processo sem condicionamento converge para um Browniano. Liggett em [Lig] estabeleceu um princípio de invariância para processos oriundos da soma condicionada de variáveis aleatórias independentes e com mesma distribuição, que é um resultado mais geral do que a obtenção da ponte Browniana.

O passo seguinte natural é tentar estender esse resultado para o modelo de Ising, porém a extensão não é imediata, já que temos que levar em consideração a existência de rugas e ilhas. Para contornar esses problemas vamos utilizar a técnica de expansấo em conglomerados ("Cluster Expansion") conjuntamente com uma representação geométrica especial da interface. Para essa representação geométrica vamos introduzir os conceitos de 
aglomerados e formas sobre esses aglomerados: formas sendo rugas e linhas verticais finitas que eventualmente ocorram em uma interface, e aglomerados sendo as projeções desses objetos sobre o eixo das abscissas. A técnica de expansão em conglomerados consiste em escrever a função de partição e valores esperados de funções nas quais tenhamos interesse numa série formal em que cada termo pode ser interpretado como sendo parte conexa (conglomerado) de um certo grafo conexo.

Com esses conceitos podemos, através da expansão de conglomerados dos contornos, interpretar o sistema, que originalmente era bidimensional, como sendo um gás de rede unidimensional de partículas representadas pelas formas sobre os aglomerados, que será chamado de gás de partículas de formas, com certos potenciais de interação e onde as partículas realizam saltos de certo tamanho dependente da forma sobre o respectivo aglomerado.

A abordagem adotada então, na prova da convergência das distribuições finitodimensionais, é diferente daquela aplicada no capítulo I, pois aqui analisamos a função característica dos saltos realizados pelas partículas e pela fórmula de inversão calculamos a distribuição. Para isso utilizamos novamente a expansão em conglomerados, porém sobre o gás de partículas de formas. Através dessa segunda expansão obtemos uma quase independência na escala macroscópica que nos permite chegar ao resultado desejado.

É importante notar que esse resultado somente é válido para temperaturas suficientemente baixas, pois a convergência das séries formais, necessária entre outras coisas para a construçấo do limite termodinâmico, só pode ser garantida nesse regime. A técnica de expansão para esse modelo específico e alguns outros modelos similares pode ser encontrada nos trabalhos de Gallavotti, Martin-Löf, Miracle-Solé ([Gal], [GMa], [GMM], [GMi]), Ruelle [Rue], Brydges [Bry] e referências nesses artigos.

O segundo capítulo exibe a técnica de expansão e sua aplicação na prova da convergência da distribuição de dimensão finita dos saltos (interface) para a da ponte Browniana, que é o resultado principal deste trabalho. Um resultado mais forte é provado por Higuchi [Hig]. Ele estabelece o mesmo resultado que obtivemos para o S.O.S., i.e., que a interface do Ising converge fracamente para a ponte Browniana. Para se chegar a esse resultado a partir do que mostramos no capítulo II, basta provar que a família de medidas de probabilidades preserva massa, o que está feito nas seçôes 4 e 5 de [Hig]. 


\section{Capítulo I}

\section{O Modelo de Interface S.O.S.}

O modelo S.O.S. (de "Solid On Solid") é, como o próprio nome diz, constituído de um sólido sobre um outro sólido. A interface é a linha de separação entre esses dois sólidos.

Este é o modelo mais simples que fornece uma descrição aproximada de uma interface separando duas fases em equilíbrio, tal como uma fase líquida e uma fase vapor, ou uma fase magnetizada positivamente e outra negativamente no modelo ferromagnético de Ising em duas dimensóes, como podemos ver na figura I.1 .
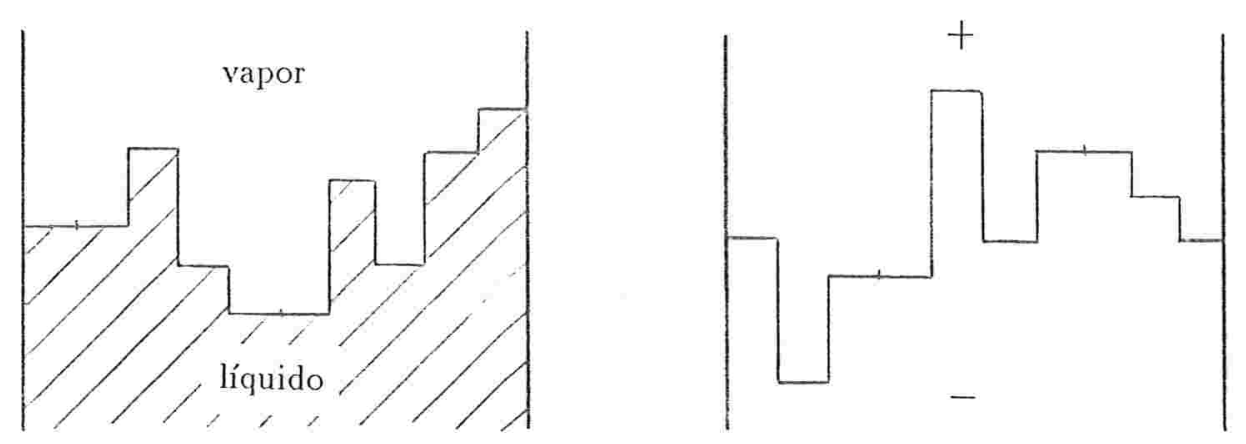

Figura I.1: Exemplos de modelos de interface tipo S.O.S.

Nesse capítulo temos como objetivo estudar o comportamento assintótico da interface e para tanto fornecemos uma descrição do modelo e provamos que, se a temperatura não for nula, a interface realmente não é lisa (reta), mas flutua. Mais do que obtermos a 
ordem da flutuação, provamos que ela flutua segundo um Browniano, quando convenientemente reescalonada. Para obtermos esse resultado apresentamos uma proposição, análoga ao teorema de Donsker, que estabelece a convergência fraca para a ponte Browniana de soma de variáveis aleatórias independentes e identicamente distribuídas condicionadas.

\section{I-1 Descrição do modelo}

Consideramos o modelo S.O.S. definido numa faixa reticulada infinita $\Lambda_{N} \times \mathbb{Z} \subset \mathbb{Z}^{2}$, onde $\Lambda_{N}=\{0,1,2, \ldots, N\} \subset \mathbb{Z}$ e $\mathbb{Z}$ representa o conjunto dos números inteiros.

Uma suposição desse modelo é que a interface é uma linha que não se auto-intercepta e nem se auto-projeta (ver figura I.2), já que a linha é obtida através da sobreposição de quadrados unitários, não podendo formar "rugas". Portanto a interface pode ser considerada como o gráfico de uma função $\eta=\left(\eta_{x}\right)_{x \in \Lambda_{N}}$ definida sobre $\Lambda_{N}$ e tomando valores em $\mathbb{Z}$, onde para cada $x \in \Lambda_{N}, \eta_{x}$ representa a altura da interface no sítio $x$ em relação à origem.
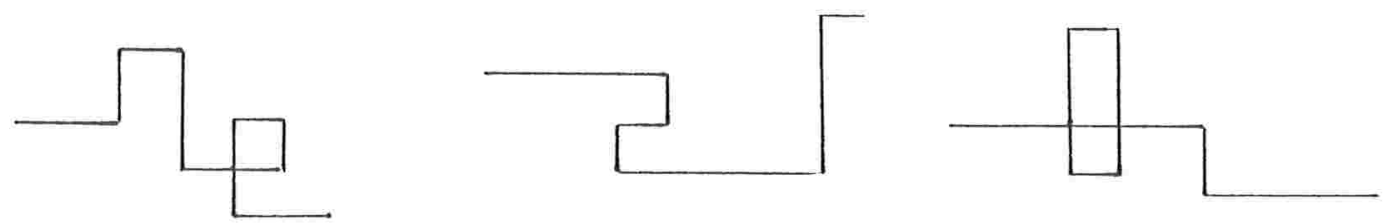

Figura I.2: Exemplos de linhas que não podem ser representadas pelo modelo S.O.S.

O espaço de estados da interface é dado por $\Omega_{N}=\left\{\eta \mid \eta: \Lambda_{N} \rightarrow \mathbb{Z}\right\}=\mathbb{Z}^{\Lambda_{N}}$.

E a medida de Gibbs $\mu_{N}$ associada a esse modelo é

$$
\mu_{N}(\eta)=\frac{\exp \left\{-\beta H_{N}(\eta)\right\}}{\sum_{\zeta \in \Omega_{N}} \exp \left\{-\beta H_{N}(\zeta)\right\}},
$$

onde $\beta$ é o inverso da temperatura e a hamiltoniana $H_{N}$ é tomada por

$$
H_{N}(\eta)=\sum_{\substack{|x-y|=1 \\ x, y \in \Lambda_{N}}}\left|\eta_{x}-\eta_{y}\right|+\sum_{\substack{|x-y|=1 \\ x \in \Lambda_{N}, y \in \partial \Lambda_{N}}}\left|\eta_{x}-\eta_{y}\right|,
$$


$\operatorname{com} \sum_{|x-y|=1}$ representando a soma sobre os vizinhos mais próximos sem repetição sobre os mesmos vizinhos e $\partial \Lambda_{N}$ é a fronteira de $\Lambda_{N}$, sendo que adotaremos as seguintes condições de fronteira:

$$
\eta_{0}=\eta_{N}=0
$$

Isso significa que as bordas (os pontos inicial e final da interface) estão presas à origem, como mostra um exemplo dado pela figura I.3 .

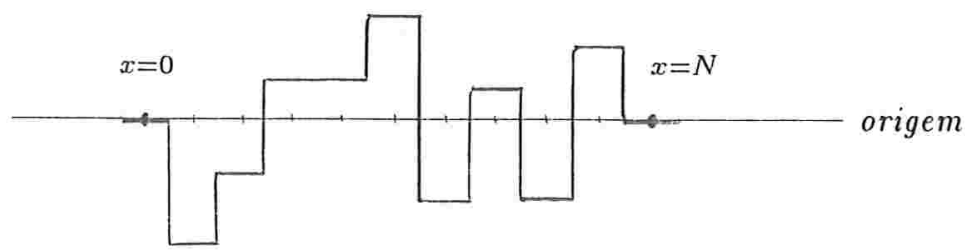

Figura I.3: A cada sítio $x$ está associada a altura $\eta_{x}$ da interface, com $\eta_{0}=\eta_{N}=0$.

Veremos que a interface do modelo, com essas condições de fronteira, tem uma flutuação da ordem de $\sqrt{N}$. Na verdade,o que obtemos é mais forte, pois é estabelecido, pelo teorema I.1 a seguir, que a interface convenientemente reescalonada comporta-se como uma Ponte Browniana em uma dimensão.

\section{I-2 Interface Browniana}

A ponte Browniana unidimensional $\mathcal{B}$ nada mais é que uma transformação do movimento Browniano unidimensional $\mathcal{W}$, dada por $\mathcal{B}_{t}=\mathcal{W}_{t}-t \mathcal{W}_{1}, 0 \leq t \leq 1$, isto é, podemos considerá-la como um movimento Browniano com ambas as bordas presas. No Apêndice A damos a definição formal do movimento Browniano e da ponte Browniana. Resultados e referências adicionais sobre Brownianos podem ser encontradas em Billingsley [Bil].

Para obtermos um comportamento dessa espécie a escala conveniente é $\sqrt{N}$, como 
comentado anteriormente. Portanto, faremos uma mudança de escala espaço-tempo, tomando as coordenadas $x \in \Lambda_{N}$ como sendo o tempo e $\eta_{x}$ a posição. Assim consideremos o seguinte processo:

$$
\eta^{N}(t) \stackrel{\text { def }}{=} \frac{1}{\sqrt{N}} \eta_{\lfloor N t\rfloor}, \quad t \in[0,1] .
$$

Este processo está definido em $D[0,1]$, que é o espaço das funções contínuas à direita e com limite à esquerda em $[0,1]$. E nesse espaço consideraremos a topologia de Skorohod (veja, por exemplo, [Bil] pg 111).

Teorema I.1 Para $0<\beta<\infty$, temos que o processo $\eta^{N}$ definido em (I.Q) converge fracamente, quando $N \rightarrow \infty$, para uma ponte Browniana, com coeficiente de difusấo $d=2 e^{-\beta} /\left(1-e^{-\beta}\right)^{2}$.

Prova: O resultado acima decorre da proposição a seguir, cuja prova se encontra na seção 3 .

Proposição I.1 (Ponte Browniana) Dada uma seqüência de variáveis aleatórias discretas $\xi_{1}, \xi_{2}, \ldots$ independentes e identicamente distribuídas no espaço $(\Omega, \mathcal{F}, P)$ com $\mathbb{E}\left(\xi_{1}\right)=0$ e $\mathbb{E}\left(\xi_{1}^{2}\right)=\sigma^{2}, 0<\sigma<\infty$; e seja $S_{n}=\xi_{1}+\xi_{2}+\ldots+\xi_{n}$ as somas parciais, definimos um elemento aleatório $X_{n}$ de $D[0,1]$ por:

$$
X_{n}(t) \stackrel{\text { not }}{=} X_{n}(t, \omega) \stackrel{\text { def }}{=} \frac{1}{\sigma \sqrt{n}} S_{\lfloor n t\rfloor}(\omega) \quad \text { para } 0 \leq t \leq 1
$$

Entâo

$$
\mathbb{E}\left(f\left(X_{n}\right) \mid X_{n}(1)=0\right) \longrightarrow \mathbb{E}(f(\mathcal{B})) \quad \text { quando } n \rightarrow \infty,
$$

para toda função limitada $f$, tal que $f: D[0,1] \rightarrow \mathbb{R}$, e $\mathcal{B}(t)$ é uma Ponte Browniana unidimensional com coeficiente de difusão $d=1$.

Deste modo, basta provar que o modelo S.O.S. satisfaz as condiçôes da proposição acima. Para isso, note que para um $\eta \in \Omega_{N}$, a variável aleatória

$$
\delta(x)=\eta_{x}-\eta_{x-1}, \quad x=1, \ldots, N,
$$


representa o salto que a interface dá entre o sítio $x-1$ e o sítio $x$, de modo que podemos escrever a altura da interface no sítio $y$ como:

$$
\eta_{y}=\sum_{x=1}^{y} \delta(x), \quad y=1, \ldots, N
$$

A partir de agora consideraremos que $\eta_{0}=0$, como no modelo original, mas deixaremos livre a borda $\eta_{N}$. A vantagem de se escrever dessa maneira é que os saltos $\{\delta(x)\}_{x \in \Lambda_{N}}$ são identicamente distribuídos e independentes como provamos a seguir:

$$
P(\delta(x)=k)=P(\eta: \delta(x)=k)=\frac{1}{\mathcal{Z}} e^{-\beta|k|} \sum_{\substack{\eta \in \Omega_{N} \\ \delta(x)=k}} \exp \left\{-\beta \sum_{\substack{y \in \Lambda_{N} \\ y \neq x}}|\delta(y)|\right\},
$$

para todo $k \in \mathbb{Z}$ e todo $x \in \Lambda_{N}$ e, $\mathcal{Z}$ sendo uma constante de normalização dada por

$$
\mathcal{Z}=\sum_{\eta \in \Omega_{N}} \exp \left\{-\beta \sum_{y \in \Lambda_{N}}|\delta(y)|\right\}
$$

De (I.3) e (I.4) temos que a distribuição dos saltos independe do sítio $x$ e é dada por:

$$
P(\delta(x)=k)=\frac{e^{-\beta|k|}}{\sum_{m \in \mathbb{Z}} e^{-\beta|m|}} .
$$

Com o intuito de provar a independência, observamos que da equação acima podemos calcular para $k, l \in \mathbb{Z}$ e para $x, y \in \Lambda_{N}$

$$
\begin{aligned}
P(\delta(x)=k, \delta(y)=l) & =\frac{1}{\mathcal{Z}} e^{-\beta(|k|+|l|)} \sum_{\substack{\eta \in \Omega_{N} \\
\delta(x)=k, \delta(y)=l}} \exp \left\{-\beta \sum_{\substack{z \in \Lambda_{N} \\
z \neq x, z \neq y}}|\delta(z)|\right\} \\
& =\frac{e^{-\beta(|k|+|l|)}}{\sum_{m \in \mathbb{Z}} \sum_{n \in \mathbb{Z}} e^{-\beta(|m|+|n|)}}=\left(\frac{e^{-\beta|k|}}{\sum_{m \in \mathbb{Z}} e^{-\beta|m|}}\right) \cdot\left(\frac{e^{-\beta|l|}}{\sum_{n \in \mathbb{Z}} e^{-\beta|n|}}\right) \\
& =P(\delta(x)=k) P(\delta(y)=l) .
\end{aligned}
$$

Analogamente, para qualquer inteiro $m, m \leq N$ temos

$$
P\left(\delta\left(x_{1}\right)=k_{1}, \ldots, \delta\left(x_{m}\right)=k_{m}\right)=\prod_{i=1}^{m} P\left(\delta\left(x_{i}\right)=k_{i}\right) .
$$


O coeficiente de difusão $d$ corresponde à variância do salto, que é finita e positiva. Logo a. expressão (I.5) nos fornece que:

$$
d=\frac{\sum_{k \in \mathbb{Z}} k^{2} e^{-\beta|k|}}{\sum_{l \in \mathbb{Z}} e^{-\beta|l|}} .
$$

Desenvolvendo o denominador da expressão (I.7) temos:

$$
\sum_{l \in \mathbb{Z}} e^{-\beta|l|}=1+2 \sum_{l=1}^{\infty}\left(e^{-\beta}\right)^{l}=\frac{1+e^{-\beta}}{1-e^{-\beta}} .
$$

E para o numerador obtemos:

$$
\begin{aligned}
\sum_{k \in \mathbb{Z}} k^{2} e^{-\beta|k|} & =2 \sum_{k=1}^{\infty} k^{2}\left(e^{-\beta}\right)^{k}=2\left\{\sum_{k=1}^{\infty} k(k-1)\left(e^{-\beta}\right)^{k}+\sum_{k=1}^{\infty} k\left(e^{-\beta}\right)^{k}\right\} \\
& =\frac{4\left(e^{-\beta}\right)^{2}}{\left(1-e^{-\beta}\right)^{3}}+\frac{2 e^{-\beta}}{\left(1-e^{-\beta}\right)^{2}} \\
& =\frac{2 e^{-\beta}}{\left(1-e^{-\beta}\right)^{2}} \frac{\left(1+e^{-\beta}\right)}{\left(1-e^{-\beta}\right)}
\end{aligned}
$$

De (I.9) e (I.8) segue que

$$
d=\frac{2 e^{-\beta}}{\left(1-e^{-\beta}\right)^{2}}
$$

Para finalizarmos a prova, basta observar que a condição de que a soma total dos saltos é zero (ou seja, $\sum_{x=1}^{N} \delta(x)=0$ ) equivale a que $\eta_{N}=0$ (sendo justamente a condição de fronteira). Dessa forma podemos aplicar a proposição I.1 e obtermos o resultado desejado.

No início do capítulo mencionamos que o modelo S.O.S. pode ser considerado como uma simplificação do modelo de Ising em duas dimensões. Em decorrência disso, é possível que tenhamos também uma flutuação de mesma ordem na interface do modelo de Ising. No próximo capítulo mostramos que isso é verdade.

\section{I-3 Prova da Proposição I.1 - Ponte Browniana}

Diferente de $\mathbb{R}^{\infty}$, em espaços de funções ou espaços métricos em geral, apenas a convergência das distribuições finito-dimensionais não garante a convergência fraca. Neces- 
sitamos de um propriedade adicional que é provar que a seqüência em questâo é relativamente (ou condicionalmente) compacta. A propriedade de preservar massa ${ }^{1}$ implica a compacidade relativa, o inverso é válido se o espaço for separável. No apêndice A apresentamos um teorema que fornece condições necessárias e suficientes para preservar massa, bem como outros teoremas clássicos da teoria das probabilidades que utilizaremos no decorrer desta prova.

Conforme [Bil], por exemplo, para provar a convergência fraca de uma seqüência de elementos aleatórios de $D[0,1]$ é suficiente provar:

(a) a convergência das distribuições finito-dimensionais e

(b) que a seqüência de distribuições preserva massa.

Estamos considerando variáveis definidas por:

$$
X_{n}(t)=\frac{1}{\sigma \sqrt{n}} S_{\lfloor n t\rfloor}=\frac{1}{\sigma \sqrt{n}} \sum_{i=1}^{\lfloor n t\rfloor} \xi_{i} \quad \text { para } t \in[0,1],
$$

onde as variáveis aleatórias $\xi_{i}$ são independentes e identicamente distribuídas com média zero e variância finita e positiva $\sigma^{2}$.

Nosso interesse reside na seqüencia $\left\{X_{n}\right\}$ condicionada ao evento $\left\{X_{n}(1)=0\right\}$. Para facilitar a notação, como $P\left(X_{n}(1)=0\right)>0$ para todo $n$, podemos definir elementos aleatórios $Y_{n}$ de $D[0,1]$ através de: $P\left(Y_{n} \in A\right)=P\left(X_{n} \in A \mid X_{n}(1)=0\right)$, para cada subconjunto mensurável $A$ de $D[0,1]$. Ou seja, para a seqüência $\left\{Y_{n}\right\}$ teremos que provar (a.) e (b).

Observe, porém, que a seqüência definida por (I.10) satisfaz o Teorema de Donsker (ver apêndice $\mathrm{A}$ ), que estabelece a convergência fraca de $X_{n}$ para o movimento Browniano $\mathcal{W}$ em $D[0,1]$. E, além disso, essa seqüência preserva massa como está provado em [Bil], por exemplo.

Por outro lado, o Teorema do Limite Local nos fornece os seguintes resultados:

$$
\sigma \sqrt{\lfloor n t\rfloor} P\left(X_{n}(t)=u\right) \stackrel{n \rightarrow \infty}{\longrightarrow} \frac{1}{\sqrt{2 \pi}} \exp \left\{-\frac{1}{2}\left(\frac{u^{2}}{t}\right)\right\}
$$

$\mathrm{e}$

$$
\sigma \sqrt{n} P\left(X_{n}(1)=0\right) \stackrel{n \rightarrow \infty}{\longrightarrow} \frac{1}{\sqrt{2 \pi}}
$$

\footnotetext{
${ }^{1}$ Preservar massa foi a tradução que adotamos para "tight".
} 
uniformemente para os pontos $u$ pertencentes ao suporte da distribuição.

Sendo assim, temos como conseqüência que:

$$
\frac{P\left(X_{n}(t)=u\right)}{P\left(X_{n}(1)=0\right)} \longrightarrow \frac{1}{\sqrt{t}} \exp \left\{-\frac{1}{2}\left(\frac{u^{2}}{t}\right)\right\}
$$

Portanto, para $0<t_{1}<t_{2}<\ldots<t_{k}<1$, considerando as distribuições finito-dimensionais de $Y_{n}$ :

$$
\begin{aligned}
& P\left(Y_{n}\left(t_{1}\right) \leq x_{1}, \ldots, Y_{n}\left(t_{k}\right) \leq x_{k}\right) \\
& =P\left(X_{n}\left(t_{1}\right) \leq x_{1}, \ldots, X_{n}\left(t_{k}\right) \leq x_{k} \mid X_{n}(1)=0\right) \\
& =\int_{-\infty}^{x_{k}} \frac{P\left(X_{n}\left(t_{1}\right) \leq x_{1}, \ldots, X_{n}\left(t_{k-1}\right) \leq x_{k-1}, X_{n}\left(t_{k}\right) \in d y, X_{n}(1)=0\right)}{P\left(X_{n}(1)=0\right)} \\
& \quad=\int_{-\infty}^{x_{k}} P\left(X_{n}\left(t_{1}\right) \leq x_{1}, \ldots, X_{n}\left(t_{k-1}\right) \leq x_{k-1}, X_{n}\left(t_{k}\right) \in d y\right) \cdot \frac{P\left(X_{n}(1)-X_{n}\left(t_{k}\right)=-y\right)}{P\left(X_{n}(1)=0\right)} .
\end{aligned}
$$

A última igualdade segue da independência de $\left\{X_{n}(1)-X_{n}\left(t_{k}\right)\right\}$ e $\left\{X_{n}\left(t_{1}\right), \ldots, X_{n}\left(t_{k}\right)\right\}$. Pelo Teorema de Donsker a medida

$$
P\left(X_{n}\left(t_{1}\right) \leq x_{1}, \ldots, X_{n}\left(t_{k-1}\right) \leq x_{k-1}, X_{n}\left(t_{k}\right) \in d y\right)
$$

converge fracamente para a medida.

$$
P\left(\mathcal{W}\left(t_{1}\right) \leq x_{1}, \ldots, \mathcal{W}\left(t_{k-1}\right) \leq x_{k-1}, \mathcal{W}\left(t_{k}\right) \in d y\right)
$$

Além disso, de (I.11) e por simetria decorre que

$$
\frac{P\left(X_{n}(1)-X_{n}\left(t_{k}\right)=-y\right)}{P\left(X_{n}(1)=0\right)} \longrightarrow \frac{1}{\sqrt{1-t_{k}}} \exp \left\{-\frac{1}{2}\left(\frac{y^{2}}{1-t_{k}}\right)\right\}
$$

pois $n-\lfloor n t\rfloor=\lfloor n(1-t)\rfloor$ ou $n-\lfloor n t\rfloor=\lfloor n(1-t)\rfloor+1$. Observamos que se $t$ for irracional então sempre teremos $n-\lfloor n t\rfloor=\lfloor n(1-t)\rfloor$.

Então (I.12) converge para

$$
\int_{-\infty}^{x_{k}} P\left(\mathcal{W}\left(t_{1}\right) \leq x_{1}, \ldots, \mathcal{W}\left(t_{k-1}\right) \leq x_{k-1}, \mathcal{W}\left(t_{k}\right) \in d y\right) \frac{1}{\sqrt{1-t_{k}}} \exp \left\{-\frac{1}{2}\left(\frac{y^{2}}{1-t_{k}}\right)\right\}
$$


que são as distribuições finito-dimensionais da ponte Browniana.

Vamos provar que $\left\{Y_{n}\right\}$ preserva massa utilizando o teorema A.3 e o fato que a seqüência $\left\{X_{n}\right\}$ preserva massa. Para esse fim, transformaremos os elementos aleatórios de $D[0,1]$ em elementos aleatórios de $D[0, t]$ para algum $t<1$. O que estamos fazendo, na realidade, é olhar o processo somente até o instante $t$. Para tanto, definimos uma aplicação $T_{t}: D[0,1] \rightarrow D[0, t]$ através de:

$$
\begin{aligned}
& \left(T_{t} x\right)(s)=x(s) \quad \text { se } s<t \quad \text { e } \\
& \left(T_{t} x\right)(t)=\lim _{s \uparrow t} x(s),
\end{aligned}
$$

para $x \in D[0,1]$

Essa transformação satisfaz o lema abaixo, que está provado em Liggett [Lig].

Lema I.1 Para um fixado $t \in(0,1)$, seja $B_{t}=\{x(\cdot) \in D[0,1] \mid x(\cdot)$ é contínua em $t\}$ e seja $\mu$ uma medida de probabilidade em $D[0,1]$ tal que $\mu\left(B_{t}\right)=1$. Então existe uma medida de probabilidade $\mu^{t}$ em $D[0, t]$ tal que $\mu^{t}(A)=\mu\left(\left(T_{t}^{-1} A\right) \cap B_{t}\right)$ para cada subconjunto mensurável $A$ de $D[0, t]$.

Além disso, dado $t \in(0,1)$, existem $M(t)$ e $n(t)$ tais que para $n \geq n(t)$ vale a desigualdade abaixo:

$$
\frac{P\left(X_{n}(1-t)=u\right)}{P\left(X_{n}(1)=0\right)} \leq M(t)
$$

para $u$ pertencente ao suporte da distribuição de $X_{n}(t)$. Essa desigualdade segue da convergência em (I.11), onde podemos tomar, por exemplo, $M(t)=\frac{1}{\sqrt{1-t}}+1$.

Para cada $n$ podemos construir elementos aleatórios $X_{n}^{t}$ e $Y_{n}^{t}$ de $D[0, t]$, a partir de $X_{n}$ e $Y_{n}$ respectivamente, da maneira descrita no lema I.1. Então, para cada subconjunto mensurável $A$ de $D[0, t]$, temos:

$$
\begin{aligned}
P\left(Y_{n}^{t} \in A\right) & =P\left(X_{n}^{t} \in\left(T_{t}^{-1} A\right) \cap B_{t} \mid X_{n}(1)=0\right) \\
& =\int_{-\infty}^{\infty} \frac{P\left(X_{n} \in\left(T_{t}^{-1} A\right) \cap B_{t}, X_{n}(t) \in d y, X_{n}(1)=0\right)}{P\left(X_{n}(1)=0\right)} \\
& =\int_{-\infty}^{\infty} P\left(X_{n} \in\left(T_{t}^{-1} A\right) \cap B_{t}, X_{n}(t) \in d y\right) \cdot \frac{P\left(X_{n}(1)-X_{n}\left(t_{k}\right)=-y\right)}{P\left(X_{n}(1)=0\right)}
\end{aligned}
$$




$$
\begin{aligned}
& \leq M(t) \int_{-\infty}^{\infty} P\left(X_{n} \in\left(T_{t}^{-1} A\right) \cap B_{t}, X_{n}(t) \in d y\right) \quad \text { para } n \geq n(t) \\
& =M(t) P\left(X_{n}^{t} \in A\right) \quad \text { para } n \geq n(t),
\end{aligned}
$$

onde na última desigualdade utilizamos a independência entre $\left\{X_{n}(1)-X_{n}(t)\right\}$ e $\left\{X_{n}(s), s \leq t\right\}$ (analogamente ao que foi feito em (I.12)) e a desigualdade (I.14).

Posto isso podemos provar a propriedade de preservar massa para a seqüência condicionada $\left\{Y_{n}\right\}$, que será feita considerando o comportamento de $\left\{Y_{n}\right\}$ no intervalo $[0, t]$ e $[t, 1]$ separadamente. No primeiro caso usaremos a desigualdade obtida em (I.15) e o Teorema A.3 e para o segundo caso é suficiente atentar ao fato que o comportamento do processo $Y_{n}$ próximo de $t=1$ é semelhante ao seu comportamento próximo de $t=0$.

Para $\varepsilon$ e $\eta>0$ dados, sendo $\mathcal{W}(0) \equiv 0$ e $\mathcal{W}(s)$ contínua em $s=0$, então $\sup _{0<r \leq s}|\mathcal{W}(r)|$ converge, com probabilidade um, para zero quando $s$ decresce para zero. $0 \leq r \leq s$

Portanto existe um irracional $t, 0<t<1 / \sqrt{2}$ tal que

$$
P\left(\sup _{s \leq t}|\mathcal{W}(s)| \geq \frac{\varepsilon}{4}\right)<\frac{\eta}{4 M(1 / \sqrt{2})}
$$

Como $\mathcal{W}$ é contínua em $t$ com probabilidade $1, X_{n}$ converge fracamente para $\mathcal{W}$ (pelo teorema de Donsker) e o funcional $\sup _{s \leq t}|x(s)|$ é contínuo, para cada $x(\cdot) \in D[0,1]$ que é contínuo em $t$ então $\sup _{s<t}\left|X_{n}(s)\right|$ converge em distribuição para $\sup _{s \leq t}|\mathcal{W}(s)|$. Logo existe um $n_{0}$ tal que para $n \geq n_{0}$

$$
P\left(\sup _{s \leq t}\left|X_{n}(s)\right| \geq \frac{\varepsilon}{4}\right)<\frac{\eta}{2 M(1 / \sqrt{2})} .
$$

E por (I.15) segue que

$$
P\left(\sup _{s \leq t}\left|Y_{n}(s)\right| \geq \frac{\varepsilon}{4}\right)<\frac{\eta}{2} \quad \text { para } n \geq \max \left\{n_{0}, n(1 / \sqrt{2})\right\} .
$$

Notemos também que para $0=s_{1}<s_{2}<\ldots<s_{k}=1$, irracionais, o vetor aleatório $\left(X_{n}\left(1-s_{1}\right), X_{n}\left(1-s_{2}\right), \ldots, X_{n}\left(1-s_{k}\right)\right)$ tem mesma distribuição que o vetor aleatório $\left(X_{n}(1)-X_{n}\left(s_{1}\right), \ldots, X_{n}(1)-X_{n}\left(s_{k}\right)\right)$. Em decorrência disso, os vetores aleatórios de $\left(Y_{n}\left(1-s_{1}\right), \ldots, Y_{n}\left(1-s_{k}\right)\right)$ e $\left(Y_{n}(1)-Y_{n}\left(s_{1}\right), \ldots, Y_{n}(1)-Y_{n}\left(s_{k}\right)\right)$ têm a mesma distribuição. 
Portanto, para $n \geq \max \left\{n_{0}, n(1 / \sqrt{2})\right\}$, obtemos que

$$
\begin{aligned}
P\left(\sup _{s_{1}, s_{2} \geq 1-t}\left|Y_{n}\left(s_{1}\right)-Y_{n}\left(s_{2}\right)\right| \geq \frac{\varepsilon}{2}\right) & \leq P\left(\sup _{s \geq 1-t}\left|Y_{n}(1)-Y_{n}(s)\right| \geq \frac{\varepsilon}{4}\right) \\
& =P\left(\sup _{s \leq t}\left|Y_{n}(s)\right| \geq \frac{\varepsilon}{4}\right) \leq \frac{\eta}{2} .
\end{aligned}
$$

De acordo com o Teorema A.3 a propriedade de preservar massa de $\left\{X_{n}\right\}$ implica a existência de números $a, \delta$ e $n_{1}$ tais que $a>\varepsilon / 2$ e $0<\delta<t$.

$$
\begin{aligned}
P\left(\sup _{s \leq 1}\left|X_{n}(s)\right|>a-\frac{\varepsilon}{2}\right) & \leq \frac{\eta}{2 M(1-t)} \quad \text { para } n \geq 1, \\
P\left(w_{X_{n}}[0, \delta) \geq \varepsilon\right) & \leq \frac{\eta}{M(1-t)} \quad \text { para } n \geq n_{1}, \quad \mathrm{e} \\
P\left(w_{X_{n}}^{\prime \prime}(\delta, 1) \geq \frac{\varepsilon}{2}\right) & \leq \frac{\eta}{2 M\left(1-\frac{t}{2}\right)} \quad \text { para } n \geq n_{1} .
\end{aligned}
$$

Os resultados acima, incluindo (I.16) e (I.17) nos permitem obter as desigualdades abaixo. Assim, para $n \geq \max \left\{n_{0}, n(1 / \sqrt{2}), n(1-t)\right\}=n^{\star}$

$$
\begin{aligned}
P\left(\sup _{s \leq 1}\left|Y_{n}(s)\right|>a\right) & \leq P\left(\sup _{s \leq 1-t}\left|Y_{n}(s)\right|>a-\frac{\varepsilon}{2}\right)+P\left(\sup _{s \geq 1-t}\left|Y_{n}(s)-Y_{n}(1-t)\right|>\frac{\varepsilon}{2}\right) \\
& \leq M(1-t) P\left(\sup _{s \leq 1-t}\left|X_{n}(s)\right|>a-\frac{\varepsilon}{2}\right)+\frac{\eta}{2} \\
& \leq \eta .
\end{aligned}
$$

Observamos que para os $n$ precedentes a $n^{\star}$, podemos assegurar a desigualdade acima tomando a crescente para cada $P_{n}, n<n^{\star}$.

Como, para cada $x(\cdot) \in D[0,1]$ temos

$$
w_{x}^{\prime \prime}(\delta, 1) \leq w_{x}^{\prime \prime}\left(\delta, 1-\frac{t}{2}\right)+\sup _{s_{1}, s_{2} \geq 1-t}\left|x\left(s_{1}\right)-x\left(s_{2}\right)\right|,
$$

então, para $n \geq \max \left\{n_{0}, n(1 / \sqrt{2}), n(1-t), n(1-t / 2)\right\}$, as desigualdades abaixo são válidas:

$$
\begin{aligned}
P\left(w_{Y_{n}}^{\prime \prime}(\delta, 1) \geq \varepsilon\right) & \leq M\left(1-\frac{t}{2}\right) P\left(w_{X_{n}}^{\prime \prime}(\delta, 1) \geq \frac{\varepsilon}{2}\right)+P\left(\sup _{s_{1}, s_{2} \geq 1-t}\left|Y_{n}\left(s_{1}\right)-Y_{n}\left(s_{2}\right)\right| \geq \frac{\varepsilon}{2}\right) \\
& \leq \eta \\
P\left(w_{Y_{n}}[0, \delta) \geq \varepsilon\right) & \leq M(1-t) P\left(w_{X_{n}}[0, \delta) \geq \varepsilon\right) \leq \eta
\end{aligned}
$$


e por simetria, como $X_{n}(0)=X_{n}(1)=0$, temos:

$$
P\left(w_{Y_{n}}[1-\delta, 1) \geq \varepsilon\right) \leq \eta
$$

Desde que (I.18), (I.19), (I.20) e (I.21) satisfazem as condições do teorema. A.3, concluímos que a seqüência $\left\{Y_{n}\right\}$ preserva massa em $D[0,1]$. E, tendo provado a convergência das distribuições finito-dimensionais, obtemos que $\left\{Y_{n}\right\}$ converge fracamente para a ponte Browniana.

Observamos que Liggett em [Lig] estabeleceu um princípio de invariância para processos que são derivados da soma de variáveis aleatórias independentes no domínio de atração de uma lei estável, condicionadas ao evento que a $n$-ésima soma parcial esteja num dado intervalo. O resultado que estabelecemos neste capítulo é um caso particular do provado em [Lig]. 


\section{Capítulo II}

\section{Interface no modelo de Ising bidimensional}

Nesse capítulo estudamos o comportamento assintótico da interface no modelo de Ising em duas dimensões, sem campo magnético externo e com determinadas condições de fronteira. Mais especificamente analisamos a sua flutuação e veremos que, no limite termodinâmico, ela é da ordem da raiz quadrada da largura da caixa em que é originalmente definido o modelo.

Observamos que agora não é tão simples se chegar a esse resultado como foi para o caso do modelo S.O.S., porque as interações não são independentes e além disso há o problema de ithas de spins opostos, como comentado na introdução deste trabalho. Assim necessitamos de ferramentas a mais que são as expansôes em conglomerados. A expansão é um ferramenta poderosa, mas faz com que o resultado fique restrito a temperaturas muito baixas, para garantir a convergência das séries.

Aplicaremos a técnica de expansão duas vezes. A primeira expansão nos possibilita. expandir o logaritmo da função de partição de uma maneira adequada a podermos interpretar o modelo, juntamente com uma representação geométrica da interface, como sendo um sistema unidimensional de um gás de partículas de formas. E, a segunda expansão nos permite analisar a flutuação da interface através da função característica do salto. 


\section{II-1 Descrição do modelo}

Para $N \geq 1$, seja $\mathcal{R}_{N}=\left\{\left(x_{1}, x_{2}\right) \in \mathbb{Z}^{2}: 0 \leq x_{1} \leq N-1,-\left\lfloor\frac{N}{2}\right\rfloor \leq x_{2} \leq\left\lfloor\frac{N-1}{2}\right\rfloor\right\}$ uma rede contida em $\mathbb{Z}^{2}$, onde $\mathbb{Z}$ é o conjunto dos números inteiros e $\lfloor u\rfloor$ representa o maior inteiro menor que $u$ e seja $\mathcal{R}_{N}^{*}=\left\{\left(x_{1}+\frac{1}{2}, x_{2}+\frac{1}{2}\right),\left(x_{1}, x_{2}\right) \in \mathcal{R}_{N}\right\}$ a rede dual.

Cada sítio $x=\left(x_{1}, x_{2}\right)$ da rede finita $\mathcal{R}_{N}^{*}$ tem um spin $\sigma_{x}$ que assume os valores -1 ou +1 . Seja $\Omega_{N}=\{-1,+1\}^{\left|\mathcal{R}_{N}^{*}\right|}$ o espaço de todas as configurações de spins. A energia do sistema de uma configuração de spin $\sigma=\left(\sigma_{x}\right)_{x \in \mathcal{R}_{N}^{*}} \in \Omega_{N}$ é dada por

$$
H_{N}(\sigma)=-\frac{1}{2} \sum_{\substack{|x-y|=1 \\ x, y \in \mathcal{R}_{N}^{*}}} \sigma_{x} \sigma_{y}-\frac{1}{2} \sum_{\substack{|x-y|=1 \\ x \in \mathcal{R}_{N}^{*}, y \in \partial \mathcal{R}_{N}^{*}}} \sigma_{x} \sigma_{y}
$$

onde a soma $\sum_{|x-y|=1}$ é tomada somente sobre os vizinhos mais próximos, sem repetição entre os mesmos vizinhos. O segundo termo da equação (II.1) considera as interaçôes dos spins situados na fronteira $\partial \mathcal{R}_{N}^{*}$ de $\mathcal{R}_{N}^{*}$.

Como pode ser observado, $H_{N}$ corresponde à energia de uma configuração de spins interagindo através de potencial de pares de vizinhos mais próximos e, também, interagindo com um conjunto de spins externos fixados, que fazem a fronteira do sistema. Esses spins da fronteira formam a condição de fronteira, neste caso dada por:

$$
\sigma_{x}=\left\{\begin{array}{ll}
+1 & \text { se } x_{2} \geq 0 \\
-1 & \text { se } x_{2}<0
\end{array} \quad \text { para todo } x=\left(x_{1}, x_{2}\right) \in \partial \mathcal{R}_{N}^{*}\right.
$$

Um quadro dessa situação é dada pela figura II.1.

Dada uma configuração de spins, considera-se a rede dual $\mathcal{R}_{N}$, e nela desenha-se um segmento unitário perpendicular ao elo entre dois spins de $\mathbb{Z}^{2}$, cada vez que esses spins tiverem sinais opostos. Esses segmentos formarão linhas que separam regiôes contendo spins de sinais opostos. O conjunto de linhas então obtido contém muitas componentes conexas (conexas no sentido que as componentes têm um ponto em comum, com a exceção dos casos em que temos a intersecçâo de 4 segmentos nos quais adotaremos uma convençâo, descrita abaixo) e cada vértice da rede $\mathcal{R}_{N}$, é a intersecção de 0,2 ou 4 segmentos com a exceçấo dos vértices $\mathrm{A} \equiv(0,0)$ e $\mathrm{B} \equiv(N, 0)$ onde há apenas a intersecção de 1 ou 3 segmentos. Os casos em que se tem um vértice da rede dual como intersecção de 4 


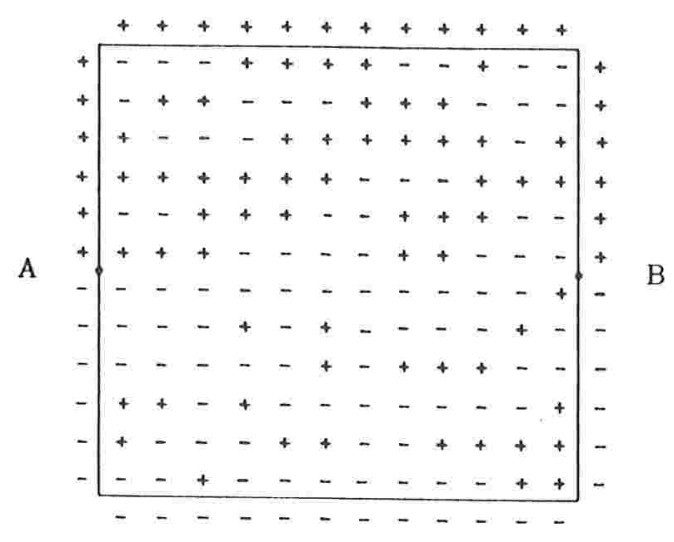

Figura II.1: Condições de fronteira

segmentos ocorrem quando temos os spins em torno do vértice como mostra a figura II.2a e, nestes casos, modificaremos levemente as linhas como mostra a figura II.2b. Isso altera o conceito de conexidade para esses casos, pois podemos ter duas componentes conexas disjuntas ao invés de apenas uma componente conexa. Esse artifício nos garante a unicidade de uma configuração de contornos para cada configuração de spins, além da unicursalidade da interface.

$$
\begin{array}{lllll}
+\mid- & - & + & +J- & -J+ \\
\hline-1+ & +1- & \frac{-1+}{+\sqrt{-}}
\end{array}
$$

(a)

Figura II.2: a. configurações que nos fornecem interseç̧ão de 4 segmentos.

b. convenção de conexidade adotada para os respectivos casos em a.

As componentes conexas serão denominadas contornos, as quais denotaremos por $\gamma_{1}, \gamma_{2}, \ldots, \gamma_{n}$ com a exceção da componente que contém os pontos A e B que será denotada por $\lambda$. Note que a componente que contém o ponto A deve também conter o ponto B e, que existe uma correspondência um a um entre configurações de contornos e configuraçôes de spins; considerando-se por configuração de contornos o conjunto $\left\{\gamma_{1}, \ldots, \gamma_{n}, \lambda\right\}$ sendo os elementos $\left(\gamma_{1}, \ldots, \gamma_{n}\right)$ fechados e $\lambda$ aberto com pontos terminais em A e B como mostra a figura II.3. 


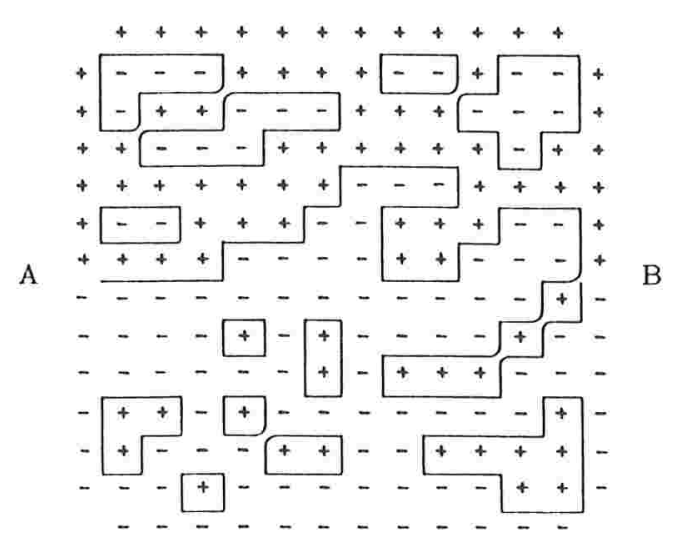

Figura II.3: A interface é a única componente conexa que vai de A a B.

Dada essa relação biunívoca de configuração de spins com configurações de contornos, é importante notar que a hamiltoniana $H_{N}$ em (II.1), que fornece a energia de uma configuração $\sigma$, pode ser expressa em termos dos comprimentos $\left|\gamma_{i}\right|,|\lambda|$ das linhas $\gamma_{i}$ e $\lambda$ a saber:

$$
H_{N}(\sigma)=\frac{1}{2} C_{N}+|\lambda|+\sum_{i=1}^{n}\left|\gamma_{i}\right|
$$

onde $C_{N}$ é o número total de interações na rede $\mathcal{R}_{N}^{*}$ e, portanto é uma constante que não depende de $\sigma$.

Para um dado valor fixado de $\beta$, onde $\beta$ é o inverso da temperatura, o ensemble grand-canônico corresponde a atribuir a uma configuração $\left\{\gamma_{1}, \ldots, \gamma_{n}, \lambda\right\}$ uma probabilidade dada pela medida de Gibbs:

$$
p_{N}(\sigma)=p_{N}\left(\gamma_{1}, \ldots, \gamma_{n}, \lambda\right)=\frac{\exp \left\{-\beta|\lambda|-\beta \sum_{i}\left|\gamma_{i}\right|\right\}}{\mathcal{Z}\left(\mathcal{R}_{N}, \beta\right)},
$$

onde o fator de normalização $\mathcal{Z}\left(\mathcal{R}_{N}, \beta\right)$ é a função de partição grand-canônica dada por:

$$
\mathcal{Z}\left(\mathcal{R}_{N}, \beta\right)=\sum_{\substack{\lambda, \gamma_{1}, \ldots, \gamma_{n} \\ \text { compativeis }}} \exp \left\{-\beta|\lambda|-\beta \sum_{i}\left|\gamma_{i}\right|\right\}
$$

e $\left\{\gamma_{1}, \ldots, \gamma_{n}, \lambda\right\}$ compativeis significa que os contornos $\gamma$ 's não têm pontos em comum entre si e nem com $\lambda$, respeitando a convenção adotada na figura II.2b.

O interesse porém, reside na linha de separação de fases ou simplesmente interface. Assim sendo, definindo $\mathcal{R}_{\lambda}$ como o conjunto de $\left\{\gamma_{1}, \ldots, \gamma_{k}\right\}$ de contornos compativeis 
para $\lambda$ fixada, a probabilidade $p_{N}(\lambda)$ de que a linha de separação de uma configuração de spins coincida com uma linha $\lambda$ dada é

$$
\begin{aligned}
p_{N}(\lambda) & =\frac{e^{-\beta|\lambda|} \sum_{\left(\gamma_{1}, \ldots, \gamma_{n}\right) \in \mathcal{R}_{\lambda}} e^{-\beta \sum_{i}\left|\gamma_{i}\right|}}{\sum_{\lambda^{\prime}} e^{-\beta\left|\lambda^{\prime}\right|} \sum_{\left(\gamma_{1}, \ldots, \gamma_{n}\right) \in \mathcal{R}_{\lambda^{\prime}}} e^{-\beta \sum_{i}\left|\gamma_{i}\right|}} \\
& =\frac{e^{-\beta|\lambda|} \mathcal{Z}_{0}\left(\mathcal{R}_{\lambda}^{(a)}, \beta\right) \mathcal{Z}_{0}\left(\mathcal{R}_{\lambda}^{(b)}, \beta\right)}{\sum_{\lambda^{\prime}} e^{-\beta\left|\lambda^{\prime}\right|} \mathcal{Z}_{0}\left(\mathcal{R}_{\lambda^{\prime}}^{(a)}, \beta\right) \mathcal{Z}_{0}\left(\mathcal{R}_{\lambda^{\prime}}^{(b)}, \beta\right)},
\end{aligned}
$$

onde

$$
\mathcal{Z}_{0}\left(\mathcal{R}_{\lambda}^{(k)}, \beta\right)=\sum_{\left(\gamma_{1}, \ldots, \gamma_{n}\right) \in \mathcal{R}_{\lambda}^{(k)}} \exp \left\{-\beta \sum_{i}\left|\gamma_{i}\right|\right\}, k=a, b
$$

representa a soma sobre as configurações de contornos acima $(k=a)$ ou abaixo $(k=b)$ de $\lambda$ que são compatíveis.

Para transformar (II.2) numa equação mais simples de se trabalhar, vamos usar a técnica de expansão em conglomerados que consiste em expandir uma função através de uma série formal, onde os termos da série são interpretados como conglomerados (componentes conexas) de um certo grafo conexo, como veremos na seção a seguir.

Um resultado que podemos obter usando argumento de Peierls e que já foi provado anteriormente por Minlos e Sinai [MS] é que, com probabilidade tendendo a 1 quando $N \rightarrow \infty$, não existe um contorno de comprimento maior que $c \log N, c>0$; e a linha $\lambda$ tem comprimento menor que $N(1+\epsilon)$, onde $\epsilon$ depende de $\beta$.

\section{II-2 Expansão em Conglomerados de Contornos}

As expansões em conglomerados aplicadas ao modelo de Ising que exibimos neste capítulo deve-se principalmente aos trabalhos de Gallavotti, Martin-Löf e Miracle-Solé ([Gal], [GMM], [GMa] e [GMi]). Um estudo mais recente sobre diversos tipos de expansões foi feito por Brydges em [Bry].

Neste trabalho apresentamos a técnica para o caso particular do modelo de Ising bidimensional seguindo a linha de Gallavotti [Gal]. Considere um espaço $\mathcal{F}$ de funções 
definidas em conjuntos finitos de contornos contidos em $\mathbb{Z}^{2}$. Os conjuntos de contornos que consideramos podem se sobrepor e mesmo coincidir, a menos de menção explícita em contrário.

Assim, sejam $\mathcal{N}=\left\{\Gamma ; \Gamma=\left(\gamma_{1}, \ldots, \gamma_{n}\right)\right\}$ uma família de conjuntos de contornos finitos e o espaço $\mathcal{F}$ de funçôes definido por:

$$
\mathcal{F}=\left\{\varphi \text { tal que } \varphi: \mathcal{N} \rightarrow \mathbb{R} ;|\varphi|_{n}=\sup _{\gamma_{1}, \ldots, \gamma_{n}}\left|\varphi\left(\gamma_{1}, \ldots, \gamma_{n}\right)\right|<\infty, \forall n \geq 0\right\}
$$

Definição III.1 Se $\varphi_{1}$ e $\varphi_{2} \in \mathcal{F}$, podemos definir seu produto de convolução, para $\Gamma \in$ $\mathcal{N}$, por:

$$
\left(\varphi_{1} * \varphi_{2}\right)(\Gamma)=\sum_{\substack{\Gamma_{1} \cup \Gamma_{2}=\Gamma \\ \Gamma_{1} \cap \Gamma_{2}=\emptyset}} \varphi_{1}\left(\Gamma_{1}\right) \varphi_{2}\left(\Gamma_{2}\right)=\sum_{\Gamma_{1} \subset \Gamma} \varphi_{1}\left(\Gamma_{1}\right) \varphi_{2}\left(\Gamma \backslash \Gamma_{1}\right)
$$

onde $\sum_{\Gamma_{1} \cup \Gamma_{2}=\Gamma}$ é considerada como uma soma de pares ordenados que decompóem $\Gamma$ em dois conjuntos de contornos (os pares $(\emptyset, \Gamma)$ e $(\Gamma, \emptyset)$ são permitidos). A soma acima é finita desde que $\Gamma$ é finito e $\left(\varphi_{1} * \varphi_{2}\right) \in \mathcal{F}$. O elemento neutro da multiplicação de $\mathcal{F}$ é dado por 1 , i.e., a identidade para o produto (II.4)

$$
\mathbb{1}(\Gamma)= \begin{cases}1 & \text { se } \Gamma=\emptyset \\ 0 & \text { se } \Gamma \neq \emptyset\end{cases}
$$

Considerando os subespaços $\mathcal{F}_{0}$ e $\mathcal{F}_{1}$ de $\mathcal{F}$ como $\mathcal{F}_{0}=\{\varphi \in \mathcal{F}: \varphi(\emptyset)=0\}$ e $\mathcal{F}_{1}=\{\varphi \in \mathcal{F}: \varphi(\emptyset)=1\}$ temos,

Definição II.2 A exponencial de uma função $\varphi \in \mathcal{F}_{0}$ é definida por

$$
(\operatorname{Exp} \varphi)(\Gamma)=\sum_{n \geq 0} \frac{\varphi^{n}(\Gamma)}{n !}=11(\Gamma)+\sum_{n \geq 1} \frac{1}{n !} \sum_{\substack{\Gamma_{1} \cup \ldots, \ldots \Gamma_{n}=\Gamma \\ \Gamma_{i} \cap \Gamma_{j}=\emptyset, i \neq j}} \varphi\left(\Gamma_{1}\right) \ldots \varphi\left(\Gamma_{n}\right)
$$

onde $\varphi^{0}(\Gamma)=11(\Gamma)$ e $\varphi^{n}$ é o produto de convolução de $n$ termos.

Essa função exponencial é uma aplicação de $\mathcal{F}_{0}$ em $\mathcal{F}_{1}$ e está bem definida já que a soma é feita sobre um conjunto finito de índices. 
Definição II.3 A função inversa da exponencial, o logaritmo, definida sobre $\mathcal{F}_{1}$ é dada por:

$$
\begin{aligned}
\left(\log \varphi_{1}\right)(\Gamma) & =(\log (11+\varphi))(\Gamma)=\sum_{n \geq 1} \frac{(-1)^{n+1}}{n} \varphi^{n}(\Gamma) \\
& =\sum_{n \geq 1} \frac{(-1)^{n+1}}{n} \sum_{\Gamma_{1} \cup \ldots \cup \Gamma_{n}=\Gamma} \varphi\left(\Gamma_{1}\right) \ldots \varphi\left(\Gamma_{n}\right),
\end{aligned}
$$

$\operatorname{com} \varphi_{1}=11+\varphi, \varphi \in \mathcal{F}_{0}$

Novamente a soma é finita e $\log \varphi_{1} \in \mathcal{F}_{0}$. Observe também que $\operatorname{Exp} \log \varphi_{1}=\varphi_{1}$ para $\varphi_{1} \in \mathcal{F}_{1}$.

A seguir definimos um produto escalar entre elementos de $\mathcal{F}$ e funções $\chi_{\theta}, \theta \subset \mathbb{Z}^{2}$, multiplicativa, isto é, $\chi_{\theta}\left(\gamma_{1}, \ldots, \gamma_{n}\right)=\chi_{\theta}\left(\gamma_{1}\right) \chi_{\theta}\left(\gamma_{2}\right) \ldots \chi_{\theta}\left(\gamma_{n}\right)$.

Definição II.4 Seja $\mathcal{D}_{\theta}=\left\{\varphi \in \mathcal{F}: \sum_{\Gamma \in \mathcal{N}}\left|\varphi(\Gamma) \chi_{\theta}(\Gamma)\right|<\infty\right\}$, então, se $\varphi \in \mathcal{D}_{\theta}$ definimos

$$
\left\langle\chi_{\theta}, \varphi\right\rangle=\sum_{\Gamma \in \mathcal{N}} \varphi(\Gamma) \chi_{\theta}(\Gamma)
$$

Com base nessas definiçôes e no fato de $\chi_{\theta}$ ser multiplicativa, obtemos que se $\varphi_{1}, \varphi_{2} \in \mathcal{D}_{\theta}$ então $\left(\varphi_{1} * \varphi_{2}\right) \in \mathcal{D}_{\theta} \mathrm{e}$

$$
\left\langle\chi_{\theta}, \varphi_{1} * \varphi_{2}\right\rangle=\left\langle\chi_{\theta}, \varphi_{1}\right\rangle\left\langle\chi_{\theta}, \varphi_{2}\right\rangle
$$

Definição II.5 Definimos uma aplicação $D_{\Gamma}: \mathcal{F} \rightarrow \mathcal{F}$ por:

$$
\left(D_{\Gamma} \varphi\right)(\Upsilon)=\varphi(\Gamma \cup \Upsilon)
$$

onde $\Gamma \cup \Upsilon=\left(\gamma_{1}, \ldots, \gamma_{n}, v_{1}, \ldots, v_{m}\right)$ se $\Gamma=\left(\gamma_{1}, \ldots, \gamma_{n}\right)$ e $\Upsilon=\left(v_{1}, \ldots, v_{m}\right)$.

A operação acima tem as seguintes propriedades:

(i) $D_{\gamma}\left(\varphi_{1} * \varphi_{2}\right)=\left(D_{\gamma} \varphi_{2}\right) * \varphi_{1}+\varphi_{2} *\left(D_{\gamma} \varphi_{1}\right)$,

(ii) $D_{\gamma}(\operatorname{Exp} \varphi)=\left(D_{\gamma} \varphi\right) *(\operatorname{Exp} \varphi)$,

(iii) $D_{\gamma}(\log \varphi)=\varphi^{-1} *\left(D_{\gamma} \varphi\right)$ para $\varphi \in \mathcal{F}_{1}$ e onde $\varphi^{-1}$ é a função inversa em relação 
a.o produto de convolução em (II.4), isto é, $\varphi^{-1} * \varphi=\varphi * \varphi^{-1}=11\left(\varphi^{-1}\right.$ está bem definida se $\varphi(\emptyset) \neq 0)$.

As provas dessas propriedades, assim como a prova de (II.5) encontram-se no apêndice B.

Com esses resultados obtemos a seguinte propriedade, cuja prova também se encontra no apêndice B:

Lema II.1 Se $\varphi \in \mathcal{F}_{0}$ e $\varphi \in \mathcal{D}_{\theta}$, então para qualquer função $\chi_{\theta}$ multiplicativa temos que $\left\langle\chi_{\theta}, \operatorname{Exp} \varphi\right\rangle=\exp \left\{\left\langle\chi_{\theta}{ }^{\prime}, \varphi\right\rangle\right\}$ ou de maneira mais explícita,

$$
\sum_{\Gamma}(\operatorname{Exp} \varphi)(\Gamma) \chi_{\theta}(\Gamma)=\exp \left\{\sum_{\Gamma} \varphi(\Gamma) \chi_{\theta}(\Gamma)\right\}
$$

Esse lema será usado $\operatorname{com} \chi_{\theta}(\Gamma)$ dada por $\chi_{\theta}(\Gamma)=\left\{\begin{array}{ll}1 & \text { se } \Gamma \subset \theta, \\ 0 & \text { se } \Gamma \not \subset \theta,\end{array}\right.$, de modo que a expressão do lema II.1 terá a seguinte forma:

$$
\sum_{\Gamma \subset \theta}(\operatorname{Exp} \varphi)(\Gamma)=\exp \sum_{\Gamma \subset \theta} \varphi(\Gamma)
$$

Com isso podemos obter uma forma mais simples para $\mathcal{Z}_{0}(\theta, \beta)$. Tendo isso em mente, consideremos uma particular função $\varphi \in \mathcal{F}_{1}$ :

$$
\varphi(\Gamma)=\left\{\begin{array}{cl}
1 & \text { se } \Gamma=\emptyset, \\
e^{-\beta \sum_{i}\left|\gamma_{i}\right|} & \text { se } \Gamma \text { consiste de contornos mutuamente } \\
& \text { compatíveis, } \Gamma=\left(\gamma_{1}, \ldots, \gamma_{n}\right), \\
0 & \text { nos outros casos. }
\end{array}\right.
$$

onde $\sum_{i=1}^{n}\left|\gamma_{i}\right|=|\Gamma|$ se $\Gamma=\left(\gamma_{1}, \ldots, \gamma_{n}\right)$ é um conjunto de contornos mutuamente compatíveis.

Para a função acima podemos definir $\varphi^{T}=\log \varphi$ de maneira que podemos escrever $\mathcal{Z}_{0}$ de (II.3) como:

$$
\mathcal{Z}_{0}(\theta, \beta)=\sum_{\Gamma \in \theta} \varphi(\Gamma)=\sum_{\Gamma \in \theta}(\operatorname{Exp} \log \varphi)(\Gamma)=\sum_{\Gamma \in \theta}\left(\operatorname{Exp} \varphi^{T}\right)(\Gamma)=\exp \sum_{\Gamma \in \theta} \varphi^{T}(\Gamma)
$$


onde a última igualdade só é válida se $\varphi^{T}(\Gamma) \in \mathcal{D}_{\theta}$, isto é, se $\sum_{\Gamma \in \theta}\left|\varphi^{T}\right|<\infty$.

É interessante notar que a razão pela qual essa técnica é denominada de expansão em conglomerados é porque para funções do tipo considerado acima, a expressão $\varphi^{T}$ pode ser escrita como função de soma de grafos conexos, que formam os conglomerados. Isso pode ser visto a partir do seguinte desenvolvimento:

Considere $\Gamma=\left(\gamma_{1}, \ldots, \gamma_{n}\right)$ um conjunto de contornos. Para $\varphi$ dada por (II.6) podemos escrever:

$$
\begin{aligned}
\varphi(\Gamma) & =\left(\operatorname{Exp} \varphi^{T}\right)(\Gamma)=\sum_{m \geq 0} \frac{1}{m !}\left(\varphi^{T}(\Gamma)\right)^{m}=\sum_{m \geq 0} \frac{1}{m !} \sum_{\Gamma_{1} \cup \ldots \cup \Gamma_{m}=\Gamma} \varphi^{T}\left(\Gamma_{1}\right) \ldots \varphi^{T}\left(\Gamma_{m}\right) \\
& =\sum_{\Gamma_{1} \cup \ldots \cup \Gamma_{m}=\Gamma}^{\prime} \varphi^{T}\left(\Gamma_{1}\right) \ldots \varphi^{T}\left(\Gamma_{m}\right)
\end{aligned}
$$

onde $\varphi^{T}\left(\Gamma_{i}\right)=\varphi^{T}\left(\gamma ; \gamma \in \Gamma_{i}\right)$ e $\sum^{\prime}$ representa a soma sobre todas as diferentes partições de $\Gamma$ em qualquer número de partes.

Por outro lado, também temos que $\varphi$ pode ser escrita como

$$
\varphi(\Gamma)=e^{-\beta \sum_{i=1}^{n}\left|\gamma_{i}\right|} \prod_{1 \leq i<j \leq n} f\left(\gamma_{i}, \gamma_{j}\right)
$$

com $f\left(\gamma, \gamma^{\prime}\right)= \begin{cases}1 & \text { se } \gamma \text { e } \gamma^{\prime} \text { são compatíveis; } \\ 0 & \text { caso contrário. }\end{cases}$

Se tomarmos $h\left(\gamma, \gamma^{\prime}\right)=f\left(\gamma, \gamma^{\prime}\right)-1$ de modo que

$$
h\left(\gamma, \gamma^{\prime}\right)=\left\{\begin{aligned}
0 & \text { se } \gamma \text { e } \gamma^{\prime} \text { são compatíveis } \\
-1 & \text { se } \gamma \text { e } \gamma^{\prime} \text { são incompatíveis , }
\end{aligned}\right.
$$

então expandindo o produto obtemos:

$$
\varphi(\Gamma)=e^{-\beta \sum\left|\gamma_{i}\right|} \prod_{1 \leq i<j \leq n}\left(1+h\left(\gamma_{i}, \gamma_{j}\right)\right)=e^{-\beta \sum\left|\gamma_{i}\right|} \sum_{C} \prod_{\left\{\gamma, \gamma^{\prime}\right\} \in C} h\left(\gamma, \gamma^{\prime}\right),
$$

onde $\sum_{C}$ denota a soma sobre todos os grafos conexos $C$ com vértices $\gamma_{1}, \ldots, \gamma_{n}$. Cada $C$ induz uma partição de $\Gamma$ em componentes conexas e pontos isolados. Se definirmos $\bar{h}\left(\Upsilon_{1}\right)$ para algum $\Upsilon \subset \Gamma$ por

$$
\bar{h}(\Upsilon)= \begin{cases}e^{-\beta \sum_{\gamma_{i} \in \Upsilon}\left|\gamma_{i}\right|} \sum_{C_{\Upsilon}} \prod_{\left\{\gamma, \gamma^{\prime}\right\} \in C_{\Upsilon}} h\left(\gamma, \gamma^{\prime}\right) & \text { se }|\Upsilon| \geq 2, \\ e^{-\beta|\gamma|} & \text { se } \Upsilon=\{\gamma\}, \\ 0 & \text { se } \Upsilon=\emptyset .\end{cases}
$$


onde a soma $\sum_{C_{\Upsilon}}$ é sobre todos os grafos conexos de $\Upsilon$, então temos que

$$
\varphi\left(\gamma_{1}, \ldots, \gamma_{n}\right)=\sum_{\Gamma_{1} \cup \ldots \cup \Gamma_{m}=\Gamma}^{\prime} \bar{h}\left(\Gamma_{1}\right) \ldots \bar{h}\left(\Gamma_{m}\right)
$$

e de (II.8) vemos que se $\Upsilon \subseteq \Gamma$

$$
\bar{h}(\Upsilon)=\varphi^{T}(\Upsilon) .
$$

Portanto, se construirmos um grafo $G$ com arestas somente entre $\gamma$ 's incompatíveis tendo peso $(-1)$, então podemos expressar $\varphi^{T}(\Gamma)$ por:

$$
\varphi^{T}\left(\gamma_{1}, \ldots, \gamma_{n}\right) \propto e^{-\beta \sum\left|\gamma_{i}\right|} \sum_{\substack{C \subset G \\ C \text { conexo }}}(-1)^{\# \text { arestas em } C}
$$

Da expressão anterior vemos que $\varphi^{T}\left(\gamma_{1}, \ldots, \gamma_{n}\right)=0$ se $\Gamma$ não é conexa, isto é, se $\left(\gamma_{1}, \ldots, \gamma_{n}\right)$ pode ser dividido em dois grupos tais que todo $\gamma$ em um dos grupos é compatível com todo $\gamma^{\prime}$ no outro. Vemos também que $\varphi^{T}$ é invariante por translação.

A expressão (II.7) permite expandir o logaritmo da função de partição em termos de $\varphi^{T}$. O fato importante desta expansão é que, como podemos observar de (II.9), $\varphi^{T}(\Gamma)$ não depende da região $\theta$. Isso nos possibilita estudar a dependência da função de partição em relação a $\theta$. Para tanto, vamos a mais algumas definições e estimativas.

Definição II.6 Para $\Gamma$ e $\Upsilon \in \mathcal{N}$ e $\varphi$ dada por (II.6) definimos:

$$
\Delta_{\Gamma}(\Upsilon)=\left\{\begin{array}{cl}
11(\Upsilon) & \text { se } \Gamma=\emptyset, \\
\left(\varphi^{-1} * D_{\Gamma} \varphi\right)(\Upsilon) & \text { se } \Gamma \neq \emptyset \text { e consiste de contornos compativeis } \\
0 & \text { nos outros casos. }
\end{array}\right.
$$

A partir da definição acima obtemos o seguinte lema:

Lema II.2 Seja $\gamma \cup \Gamma$ um conjunto de contornos compatíveis. Então, podemos escrever a seguinte equaçâo para $\Delta_{\gamma \cup \Gamma}(\Upsilon)$ para $\Upsilon$ arbitrário

$$
\Delta_{\gamma \cup \Gamma}(\Upsilon)=e^{-\beta|\gamma|} \sum_{\substack{P \subset \Upsilon \\ P \cap \gamma \neq \emptyset}}(-1)^{N(P)} \Delta_{\Gamma \cup P}(\Upsilon \backslash P),
$$


onde a soma é sobre todos os subconjuntos $P$ de $\Upsilon$ (inclusive o vazio) tal que todos os contornos em $P$ interseptam $\gamma$ e $\Gamma \cup P$ é um conjunto de contornos compativeis $\left(\Delta_{\Gamma}(\Upsilon)=\right.$ 0 se $\Gamma$ tem contornos incompatíveis, como pode ser visto da definição II.6) e $N(P)$ é o número de contornos em $P$.

A prova desse lema está feita no apêndice B deste trabalho.

Como queremos mostrar que a expressão (II.7) é válida, temos que provar que $\varphi^{T} \in$ $\mathcal{D}_{\theta}$. Entretanto, observemos que

$$
\varphi^{T}(\gamma \cup \Gamma)=D_{\gamma} \varphi^{T}(\Gamma)=\left(D_{\gamma} \log \varphi\right)(\Gamma) \stackrel{\text { prop (iii) }}{=}\left(\varphi^{-1} *\left(D_{\gamma} \varphi\right)\right)(\Gamma) \stackrel{\text { def II.6 }}{=} \Delta_{\gamma}(\Gamma)
$$

Vamos então estudar um pouco mais a função $\Delta$, considerando a seguinte função:

$$
I_{m}=\sup _{\substack{\gamma_{1}, \ldots, \gamma_{n} \\ m \geq n \geq 1}} \sum_{\substack{\Upsilon \\ N(\Upsilon)=m-n}}\left|\Delta_{\gamma_{1}, \ldots, \gamma_{n}}(\Upsilon)\right| \exp \left\{\frac{\beta}{2} \sum_{i=1}^{n}\left|\gamma_{i}\right|\right\} .
$$

A partir do lema II.2 podemos calcular, para $N(\Gamma) \leq m$

$$
\begin{aligned}
& \sum_{\substack{\Upsilon \\
N(\Upsilon)+N(\Gamma)=m}}\left|\Delta_{\gamma \cup \Gamma}(\Upsilon)\right| e^{\frac{\beta}{2}(|\gamma|+|\Gamma|)} \leq \sum_{\substack{\Upsilon \\
N(\Upsilon)+N(\Gamma)=m}} \sum_{\substack{P \subset \Upsilon \\
P \cap \gamma \neq \emptyset}}\left|\Delta_{\Gamma \cup P}(\Upsilon \backslash P)\right| e^{\frac{\beta}{2}(|\gamma|+|\Gamma|)-\beta|\gamma|} \\
& \leq \sum_{\frac{P}{P \cap \gamma \neq \emptyset}} I_{m} \exp \left\{-\frac{\beta}{2}(|\gamma|+|P|)\right\}=I_{m} e^{-\frac{\beta|\gamma|}{2}} \sum_{P} e^{-\frac{\beta}{2}|P|} \\
& \leq I_{m} e^{-\frac{\beta|\gamma|}{2}} \exp \left\{\sum_{\frac{P}{P \cap \gamma \neq \emptyset}} e^{-\frac{\beta|P|}{2}}\right\}=I_{m} e^{-\frac{\beta|\gamma|}{2}} \exp \left\{\sum_{v \cap \gamma \neq \emptyset} e^{-\frac{\beta|v|}{2}}\right\} \\
& \leq I_{m} e^{-\frac{\beta|\gamma|}{2}} \exp \left\{\sum_{p \in \gamma} \sum_{v \ni p} e^{-\frac{\beta|v|}{2}}\right\} \leq I_{m} e^{-\frac{\beta|\gamma|}{2}} \exp \left\{|\gamma| \sum_{l=4}^{\infty}\left(3 e^{\left.-\frac{\beta}{2}\right)^{l}}\right\}\right. \\
&=I_{m} \exp \left\{|\gamma|\left(-\frac{\beta}{2}+\frac{3^{4} e^{-2 \beta}}{\left(1-3 e^{-\frac{\beta}{2}}\right)}\right)\right\}
\end{aligned}
$$

onde a última igualdade é válida se $3 e^{-\beta / 2}<1$. Usamos também o fato que o número de contornos diferentes de comprimento $l$, que passa num ponto fixado, é menor que $3^{l}$ (argumento de Peierls). 
Como $|\gamma| \geq 4$ e se considerarmos $3 e^{-\beta / 2}<1 / 2$, então o lado direito de (II.12) é menor que $I_{m} e^{-\beta}$, de onde concluímos que

$$
I_{m+1} \leq I_{m} e^{-\beta} \quad \text { se } \beta>2 \ln 6
$$

Além disso, de

$$
\begin{aligned}
I_{1} & =\sup _{\gamma}\left|\Delta_{\gamma}(\emptyset)\right| e^{\frac{\beta}{2}|\gamma|}=\sup _{\gamma}\left|\left(\varphi^{-1} * D_{\gamma} \varphi\right)(\emptyset)\right| e^{\frac{\beta}{2}|\gamma|} \\
& =\sup _{\gamma}|\varphi(\gamma)| e^{\frac{\beta}{2}|\gamma|}=\sup _{l \geq 4} e^{-\frac{\beta}{2}|l|}=e^{-2 \beta}
\end{aligned}
$$

segue de (II.13) que:

$$
I_{m} \leq e^{-\beta(m+1)}, \quad \text { para } m \geq 1 \text { e } \beta>2 \ln 6
$$

Proposição II.1 A partir do lema II.2 e das expressões (II.10), (II.11) e (II.14) chegase aos resultados abaixo. Denotamos por $p \in \Gamma$ quando o ponto $p$ da rede é vértice de algum contorno de $\Gamma$.

(1) Existe uma função $\delta(\beta)$ que tende a zero exponencialmente rápido quando $\beta \rightarrow \infty$ tal que: $\sum_{\Gamma \ni p}\left|\varphi^{T}(\Gamma)\right| \leq \delta(\beta)$.

(2) Existe uma função $\varpi(\beta)$ tendendo a zero exponencialmente rápido quando $\beta \rightarrow \infty$ tal que: $\sum_{\substack{\Gamma \ni \gamma \\ N(\Gamma)=n+1}}\left|\varphi^{T}(\Gamma)\right| \leq \varpi(\beta)^{n+1} e^{-\frac{1}{2} \beta|\gamma|}$.

(3) Se $p$ é um vértice da rede e $Q$ é um conjunto de vértices da rede a uma distância $d(p, Q)$ de $p$ então, para $\beta>\beta_{0}>0$

$$
\sum_{\substack{\Gamma \ni p \\ \Gamma \cap Q \neq \emptyset}}\left|\varphi^{T}(\Gamma)\right| \leq \delta\left(\beta_{0}\right) e^{-\left(\beta-\beta_{0}\right) d}
$$

(4) Se $Y=\left(y_{1}, \ldots, y_{m}\right)$ um conjunto de vértices distintos da rede e definimos $\Phi_{0}(Y)=\sum_{\Gamma \ni Y^{\prime}}\left|\varphi^{T}(\Gamma)\right|$, onde $\Gamma \ni Y$ significa que $\left(y_{1}, \ldots, y_{m}\right)$ são vértices dos contornos em $\Gamma$, então temos que: 
- $\psi(\beta)=\sum_{Y \ni 0} \Phi_{0}(Y)<\infty$ onde $\psi(\beta) \stackrel{\beta \rightarrow \infty}{\longrightarrow} 0$ exponencialmente.

$\sum_{Y \ni 0, \operatorname{diam} Y \geq r} \Phi_{0}(Y) \leq R_{0}^{-r} \psi(\beta) \operatorname{com} R_{0}=100$.

A prova da proposição acima se encontra no apêndice B.

A importância da proposição acima reside nos fatos que obtemos que $\sum\left|\varphi^{T}(\Gamma)\right|$ é somável de modo que o lema II.1 se aplica para $\varphi$ dada por (II.6), e que essas desigualdades nos permitem construir o limite termodinâmico.

Assim sendo, a expressão (II.7) é válida de modo a obtermos:

$$
\frac{\mathcal{Z}_{0}\left(\mathcal{R}_{\lambda}^{(a)}, \beta\right) \mathcal{Z}_{0}\left(\mathcal{R}_{\lambda}^{(b)}, \beta\right)}{\mathcal{Z}_{0}\left(\mathcal{R}_{\lambda}, \beta\right)}=\exp \left\{-\sum_{\substack{\Gamma i \lambda \\ \Gamma \subset \mathcal{R}_{N}}} \varphi^{T}(\Gamma)\right\}
$$

onde $\mathcal{Z}_{0}\left(\mathcal{R}_{\lambda}^{(k)}, \beta\right)$ está definido em (II.3) e $\Gamma i \lambda$ significa que $\{\Gamma\} \cap \lambda \neq \emptyset$. Isso nos permite escrever a distribuição de probabilidade (II.2) como sendo:

$$
p_{N}(\lambda)=\frac{\exp \left\{-\beta|\lambda|-\sum_{\substack{\Gamma i \lambda \\ \Gamma \subset \mathcal{R}_{N}}} \varphi^{T}(\Gamma)\right\}}{\text { normalização }} .
$$

Para estudar a flutuação de $\lambda$ vamos introduzir os conjuntos abaixo:

$$
\begin{aligned}
I_{N}= & \left\{x=\left(x_{1}, x_{2}\right) \in \mathbb{Z}^{2}: 0 \leq x_{1} \leq N-1\right\}, \\
\hat{\Lambda}_{N}= & \left\{\lambda \subset I_{N}: \text { o comprimento }|\lambda| \text { de } \lambda \text { é finito, } \lambda \ni(0,0)=A\right. \text { e existe um } \\
& \text { ponto } \mathrm{B}^{\prime} \in\{(N, j) ; j \in \mathbb{Z}\} \subset I_{N} \text { tal que } \lambda \text { pode ser considerada como } \\
& \text { uma curva de } \left.\mathrm{A} \mathrm{a} \mathrm{B}^{\prime}\right\} \\
\text { e } \Lambda_{N}= & \left\{\lambda(\eta) ; \eta \in \Omega_{N}\right\}=\left\{\lambda \in \hat{\Lambda}_{N} ; \lambda \subset \mathcal{R}_{N}\right\},
\end{aligned}
$$

onde $\lambda \subset \hat{\Lambda}_{N}$ significa que $\lambda$ é uma linha conexa definida na rede $I_{N}$ através do mesmo procedimento da seção 1 .

A razão de considerarmos o conjunto $\hat{\Lambda}_{N}$ é no sentido de fazer uma analogia ao capítulo I, "soltando" a ponta final (o ponto B) da linha e depois condicionar a que essa 
ponta final seja o ponto B. Note que em $\hat{\Lambda}_{N}$ está embutida a idéia de que $\lambda$ é unicursal (ver figura II.3).

Assim, se $\lambda \in \Lambda_{N}$, temos a distribuição de probabilidade dada por (II.15), mas para $\lambda \in \hat{\Lambda}_{N}$ teremos:

$$
\hat{p}_{N}(\lambda) \propto e^{-\beta|\lambda|} \exp \left\{-\sum_{\substack{\Gamma i \lambda \\ \Gamma \subset I_{N}}} \varphi^{T}(\Gamma)\right\} .
$$

Para reduzir $\hat{\Lambda}_{N}$ a um sistema de partícula unidimensional vamos introduzir os conceitos de aglomerados e formas, dando uma representação geométrica de $\lambda$.

\section{II-3 Representação Geométrica de $\lambda$}

A fim de formalizar a estrutura de uma linha $\lambda \in \hat{\Lambda}_{N}$ arbitrária definimos duas seqüências de inteiros $\left\{\tau_{\nu}^{N}\right\}_{\nu=1}^{\kappa(\lambda)},\left\{\tilde{\tau}_{\nu}^{N}\right\}_{\nu=1}^{\kappa(\lambda)}$ dependendo de $\lambda$ através de

$$
\begin{aligned}
& \tau_{1}^{N}=\left\{\begin{array}{l}
\min \left\{j: 0 \leq j \leq N, j \in \mathbb{Z} ; \#\left\{\lambda \cap q_{j}\right\} \geq 2\right\} \\
\infty \text { se o conjunto acima é vazio. }
\end{array}\right. \\
& \tilde{\tau}_{1}^{N}=\left\{\begin{array}{l}
\min \left\{j: \tau_{1}^{N} \leq j, j \in \mathbb{Z} ; \#\left\{\lambda \cap q_{j+1 / 2}\right\}=1\right\} \\
N \text { se } \tau_{1}^{N}<\infty \text { e o conjunto acima é vazio. } \\
\infty \text { se } \tau_{1}^{N}=\infty .
\end{array}\right. \\
& \vdots \\
& \tau_{\nu}^{N}=\left\{\begin{array}{l}
\min \left\{j: \tilde{\tau}_{\nu-1}^{N}<j, j \in \mathbb{Z} ; \#\left\{\lambda \cap q_{j}\right\} \geq 2\right\} \\
\infty \text { se o conjunto acima é vazio. }
\end{array}\right. \\
& \tilde{\tau}_{\nu}^{N}=\left\{\begin{array}{l}
\min \left\{j: \tau_{\nu}^{N} \leq j, j \in \mathbb{Z} ; \#\left\{\lambda \cap q_{j+1 / 2}\right\}=1\right\} \\
N \text { se } \tau_{\nu}^{N}<\infty \text { e o conjunto acima é vazio. } \\
\infty \text { se } \tau_{\nu}^{N}=\infty .
\end{array}\right.
\end{aligned}
$$

onde $q_{j}$ são linhas verticais passando por $j \in \mathbb{Z}, \kappa(\lambda)=\operatorname{máx}\left\{\nu ; \tau_{\nu}^{N}<\infty, \tilde{\tau}_{\nu}^{N}<\infty\right\}$ e $\#\left\{\lambda \cap q_{j}\right\} \in \mathbb{N} \cup\{\infty\}$ é o número de vezes que $\lambda$ intersepta $q_{j}$.

Para cada $1 \leq \nu \leq \kappa(\lambda)$ chamamos o conjunto $\xi_{\nu}=\left\{j \in \mathbb{Z} ; \tau_{\nu}^{N} \leq j \leq \tilde{\tau}_{\nu}^{N}\right\}$ de um aglomerado de $\lambda$; isto é, um aglomerado consiste de $(k+1)$ pontos consecutivos 
$\left(q_{0}, q_{0}+1, \ldots, q_{0}+k\right), k \geq 0 ;$ em cujos pontos as linhas verticais $q_{j}, 0 \leq j \leq N(j \in \mathbb{Z})$ interceptam $\lambda$ em mais que um ponto. Além disso $\kappa(\lambda)$ é o número de aglomerados determinado por $\lambda$ e, a forma $\mathcal{S}_{\xi_{\nu}}$ sobre $\xi_{\nu}$ é o conjunto:

$$
\mathcal{S}_{\xi_{\nu}}=\lambda \cap\left\{\bigcup_{\substack{\alpha \in R \\ \tau_{\nu}^{N} \leq \alpha \leq \tilde{\tau}_{\nu}^{N}}} q_{\alpha}\right\} .
$$

Para um aglomerado $\xi$ fixado, duas formas $\mathcal{S}_{\xi}$ e $\mathcal{S}_{\xi}^{\prime}$ são consideradas semelhantes se uma delas é simplesmente uma translação da outra.

Fixada uma linha $\lambda$, já temos $\mathrm{A}=(0,0)$ como ponto inicial e $\mathrm{B}^{\prime}$ como ponto final. Considerando a unicursalidade de $\lambda$ podemos definir a entrada e saída de cada $\mathcal{S}_{\xi}$, de maneira que cada $\lambda \in \hat{\Lambda}_{N}$ é representada por $\left(\xi_{1}, \ldots, \xi_{\kappa(\lambda)} ; \mathcal{S}_{\xi_{1}}, \ldots, \mathcal{S}_{\xi_{\kappa(\lambda)}}\right)$. Reciprocamente, dada uma coleção de aglomerados distintos $\left(\xi_{1}, \ldots, \xi_{s}\right)$ e suas formas $\left(\mathcal{S}_{\xi_{1}}, \ldots, \mathcal{S}_{\xi_{s}}\right)$ com suas entradas e saídas, existe correspondentemente uma única curva $\lambda \in \hat{\Lambda}_{N}$. De modo que temos uma relação biunívoca entre $\lambda$ e aglomerados com suas formas. Assim, $\lambda=\left(\xi_{1}, \ldots, \xi_{\kappa(\lambda)} ; \mathcal{S}_{\xi_{1}}, \ldots, \mathcal{S}_{\xi_{\kappa(\lambda)}}\right)$.

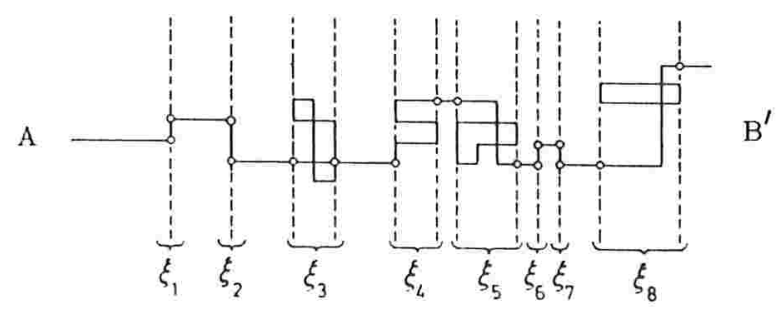

Figura II.4: Representação da interface através dos aglomerados e formas.

Para ter uma idéia clara dessa representação geométrica veja a figura II.4. A linha $\lambda$ assim representada pode ser interpretada como uma configuração de um gás de rede unidimensional com múltiplos componentes, onde as partículas do gás de rede são tantas quantos forem os aglomerados; ou seja existe a restrição de que o número de ocupação é no máximo 1, o que pró́be a sobreposição dos aglomerados.

Definição II.7 Para cada $\left(\xi_{\nu}, \mathcal{S}_{\xi_{\nu}}\right)$ associamos alguns parâmetros definidos abaixo:

(i) $\delta \mathcal{S}_{\xi_{\nu}}=\left(\right.$ altura da saída) - (altura da entrada) $=($ salto $) ; \delta \mathcal{S}_{\xi_{\nu}}=0, \pm 1, \pm 2, \ldots$ 
(ii) $\left|\xi_{\nu}\right|=\tau_{\nu}^{N}-\tilde{\tau}_{\nu}^{N}=$ comprimento do aglomerado;

(iii) $\left|\mathcal{S}_{\xi_{\nu}}\right|=$ (comprimento total de $\left.\mathcal{S}_{\xi_{\nu}}\right)-\left|\xi_{\nu}\right|=$ excesso do comprimento de $\left(\xi_{\nu}, \mathcal{S}_{\xi_{\nu}}\right)$ $=$ tamanho da forma $\mathcal{S}_{\xi_{\nu}}$.

Assim temos que

$$
|\lambda|=N+\sum_{\nu=1}^{\kappa(\lambda)}\left|\mathcal{S}_{\xi_{\nu}}\right|
$$

Claramente existe uma correspondência biunívoca entre as configurações do gás de rede unidimensional e as linhas $\lambda \in \hat{\Lambda}_{N}$.

A seguir mostramos que o problema de estudar a distribuição de probabilidade de $\lambda$ (ou seja a ordem da flutuação) é equivalente ao problema de estudar o estado de equilíbrio de um gás de rede unidimensional com múltiplos componentes (com certas atividades) sob a influência de potenciais de interação de muitos corpos.

\section{II-4 Potencial do Gás de Formas}

Como comentado anteriormente, se $\lambda \in \hat{\Lambda}_{N}, \lambda=\left(\xi_{1}, \ldots, \xi_{n} ; \mathcal{S}_{\xi_{1}}, \ldots, \mathcal{S}_{\xi_{n}}\right)$, onde $X=$ $\left(\xi_{1}, \ldots, \xi_{n}\right)$ é um conjunto de aglomerados disjuntos, então $\mathcal{S}_{X}=\left(\mathcal{S}_{\xi_{1}}, \ldots, \mathcal{S}_{\xi_{n}}\right)$ pode ser interpretado como uma configuração de um gás com múltiplos componentes em [A,B]. Observamos que se $X=T \cup T^{\prime}$ e $T \cap T^{\prime}=\emptyset$ então podemos escrever $\mathcal{S}_{X}=\mathcal{S}_{T \cup T^{\prime}}=$ $\mathcal{S}_{T} \cup \mathcal{S}_{T^{\prime}}$, e denotaremos $\left|\mathcal{S}_{X}\right|=\sum_{i}\left|\mathcal{S}_{\xi_{i}}\right|$.

Assim, cada $\mathcal{S}_{\xi}$ é uma partícula com atividade (fugacidade) $z_{\mathcal{S}_{\xi}}=e^{-\beta\left|\mathcal{S}_{\xi}\right|}$. Resta obter os potenciais de interação. A estratégia a seguir é interpretar a quantidade $\left\{-\sum_{\Gamma i \lambda} \varphi^{T}(\Gamma)\right\}$, que ocorre em (II.12) como sendo uma energia potencial associada a uma configuração $\mathcal{S}_{X}$, escrevendo-a na forma:

$$
\sum_{\Gamma i \lambda} \varphi^{T}(\Gamma)=\sum_{\Gamma i[\mathrm{~A}, \mathrm{~B}]} \varphi^{T}(\Gamma)+U\left(\mathcal{S}_{X}\right)
$$

onde $U\left(\mathcal{S}_{X}\right)$ (para a definiçôes precisas vide abaixo) representa a contribuição total da 
interação de muitos corpos, ou seja $U\left(\mathcal{S}_{X}\right)$ é da forma:

$$
U\left(\mathcal{S}_{X}\right)=\sum_{T \subset X} \Phi\left(\mathcal{S}_{T}\right)
$$

onde $\Phi\left(\mathcal{S}_{T}\right)$ representa os potenciais de interação de $n$-corpos associados ao conjunto $\mathcal{S}_{T}$ de formas, definidos pela expressão:

$$
\Phi\left(\mathcal{S}_{T}\right)=(-1)^{N(T)} \sum_{Y \subset T}(-1)^{N(Y)} U\left(\mathcal{S}_{Y}\right)
$$

onde $N(Y)$ representa o número de aglomerados em $Y$ e consideramos $\Phi(\emptyset)=0$.

Os potenciais $\Phi\left(\mathcal{S}_{T}\right)$ serão chamados de potenciais do gás de formas e verificam o seguinte lema:

Lema II.3 Se $\beta$ é suficientemente grande, os potenciais de formas verificam a seguinte desigualdade:

$$
\left|\Phi\left(\mathcal{S}_{X}\right)\right| \leq \Phi_{0}(X)\left|\mathcal{S}_{\xi}\right|
$$

onde $\xi \in X$ é o primeiro ou o último aglomerado de $X$. Além disso, $\Phi_{0}(X)$ é uma função invariante por translação de $X$ e

$$
\sup _{\xi_{0}, T} \sum_{\substack{X \ni \xi_{0} \\ X \subset T}} \Phi_{0}(X)=\psi(\beta)
$$

onde o sup é tomado sobre as configuraçôes $T$ de aglomerados permitidos (i.e., sem sobreposição) que contém $\xi_{0}$. A função $\psi(\beta) \mathrm{em}$ (II.22) tende a zero exponencialmente rápido quando $\beta$ tende ao infinito. $(\psi(\beta)$ pode ser tomada como em (4) da proposição II.1). E para $R_{0}=100$ :

$$
\sum_{\substack{X \ni \xi_{0} \\ X \ni \xi_{1}}} \Phi_{0}(X) \leq 24\left(8 R_{0}^{-1}\right)^{d\left(\xi_{0}, \xi\right)} \psi(\beta)
$$

onde $d\left(\xi_{0}, \xi\right)=$ distância entre $\xi_{0} e \xi$.

Daremos aqui um esboço da construçào de $U\left(\mathcal{S}_{X}\right)$. A construção completa se encontra em [Gal], apêndice 3 .

Lembramos que estamos tratando apenas de conjuntos de aglomerados contidos em $I_{N}$. Omitiremos a referência a isso para não sobrecarregar a notação, mas sempre que nos referirmos a algum $\Gamma$, nesta seção, deve estar implícito que $\Gamma \subset I_{N}$. 
A idéia básica é dividir o conjunto $\{\Gamma i \lambda\}$ em três classes de conjuntos $C_{1}(\lambda), C_{2}(\lambda)$ e $C_{3}(\lambda)$, para cada configuração $\lambda=\left(\xi_{1}, \ldots, \xi_{s} ; \mathcal{S}_{\xi_{1}}, \ldots, \mathcal{S}_{\xi_{s}}\right)$. Essas classes são definidas por:

$$
\begin{aligned}
C_{1}(\lambda) & =\left\{\Gamma i \lambda, \Gamma \bigcap \bigcup_{i=1}^{s} \mathcal{S}_{\xi_{i}}=\emptyset, \Pi_{[\mathrm{A}, \mathrm{B}]}^{\Gamma} \cap \bigcup_{i=1}^{s} \xi_{i}=\emptyset\right\}, \\
C_{2}(\lambda) & =\left\{\Gamma i \lambda, \Gamma \bigcap \bigcup_{i=1}^{s} \mathcal{S}_{\xi_{i}} \neq \emptyset\right\} \mathrm{e} \\
C_{3}(\lambda) & =\left\{\Gamma i \lambda, \Gamma \bigcap \bigcup_{i=1}^{s} \mathcal{S}_{\xi_{i}}=\emptyset, \Pi_{[\mathrm{A}, \mathrm{B}]}^{\Gamma} \cap \bigcup_{i=1}^{s} \xi_{i} \neq \emptyset\right\},
\end{aligned}
$$

onde $\Pi_{[\mathrm{A}, \mathrm{B}]}^{\Gamma}$ é a projeção de $\Gamma$ sobre o segmento $[\mathrm{A}, \mathrm{B}]$.

Consideremos também, a classe $C_{1}^{\prime}\left(\xi_{1}, \ldots, \xi_{s}\right)$ dos conjuntos $\Gamma$ que interseptam o segmento $[A, B]$ e cuja projeção sobre $[A, B]$ se sobrepõe com algum dos aglomerados $\xi_{1}, \ldots, \xi_{s}$, ou seja,

$$
C_{1}^{\prime}\left(\xi_{1}, \ldots, \xi_{s}\right)=\left\{\Gamma i[\mathrm{~A}, \mathrm{~B}], \Pi_{[\mathrm{A}, \mathrm{B}]}^{\Gamma} \cap \bigcup_{i=1}^{s} \xi_{i} \neq \emptyset\right\} .
$$

Essas definições nos permitem escrever

$$
\begin{aligned}
\sum_{\Gamma i \lambda} \varphi^{T}(\Gamma) & =\sum_{\Gamma \in C_{1}(\lambda)} \varphi^{T}(\Gamma)+\sum_{\Gamma \in C_{2}(\lambda)} \varphi^{T}(\Gamma)+\sum_{\Gamma \in C_{3}(\lambda)} \varphi^{T}(\Gamma) \\
= & \sum_{\Gamma i[A, B]} \varphi^{T}(\Gamma)-\sum_{\Gamma \in C_{1}^{\prime}\left(\xi_{1}, \ldots, \xi_{s}\right)} \varphi^{T}(\Gamma)+\sum_{\Gamma \in C_{2}(\lambda)} \varphi^{T}(\Gamma)+\sum_{\Gamma \in C_{3}(\lambda)} \varphi^{T}(\Gamma) .
\end{aligned}
$$

Observamos que a soma em $\Gamma \in C_{1}$ equivale às duas primeiras somas do lado direito de (II.24) se estamos considerando os contornos em $I_{N}$. Se considerarmos os contornos em $\mathcal{R}_{N}$ haverá um resto que desaparece exponencialmente, pelas desigualdades da proposição II.1, quando tomamos o limite de $N$ tendendo a infinito na direção vertical.

De maneira que (II.18) equivale às três últimas somas em (II.24) denotadas por $U\left(\mathcal{S}_{X}\right)$. Observamos que cada uma dessas somas pode ser escrita da forma $\sum_{T \subset X} \Phi^{(i)}\left(\mathcal{S}_{T}\right)$, onde $i=1,2,3$ corresponde, respectivamente, às somas em $C_{1}^{\prime}, C_{2}$ e $C_{3}$. Além disso, cada uma das funções $\Phi^{(i)}$ verificam o lema II.3. 
Mostramos a afirmação acima para a soma em $C_{1}^{\prime}$, usando o princípio da inclusãoexclusão, que fornece

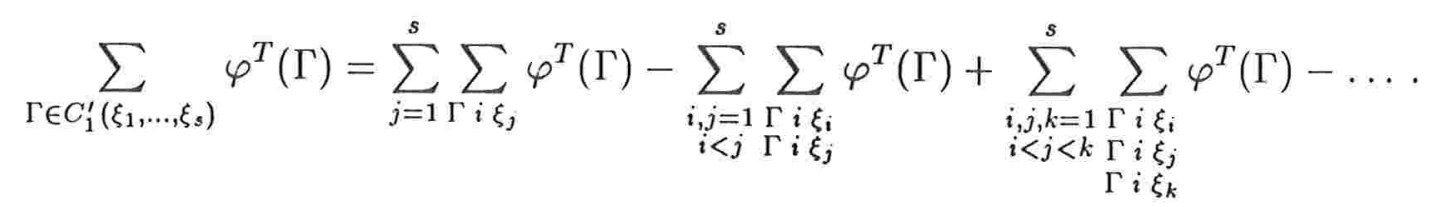

considerando $\Phi^{(1)}$ como

$$
\Phi^{(1)}\left(\mathcal{S}_{\xi_{1}} \cup \mathcal{S}_{\xi_{2}} \cup \ldots \cup \mathcal{S}_{\xi_{n}}\right)=(-1)^{n+1} \sum_{\substack{\Gamma_{i} \xi_{j} \\ j=1, \ldots n}} \varphi^{T}(\Gamma), \quad n=1, \ldots, s .
$$

Um raciocínio similar, mas que demanda mais algumas definições e cálculos, fornece o resultado acima para as outras somas, e usando o resultado (4) da proposição II.1 com o fato que $\left|\mathcal{S}_{\xi}\right| \geq(|\xi|+1)$, temos que:

$$
\left|\Phi^{(1)}\left(\mathcal{S}_{X}\right)\right| \leq\left|\mathcal{S}_{\xi^{\star}}\right| \psi(\beta) R_{0}^{-\delta(X)}\left|\mathcal{S}_{\xi^{\star}}\right| \Phi_{0}^{(1)}(X),
$$

onde $\delta(X)=$ distância entre o primeiro e o último aglomerado de $X$ e $\xi^{\star}$ é o primeiro ou o último aglomerado de $X$.

Portanto se $T$ é um conjunto de aglomerados compatíveis:

$$
\sum_{\substack{X \ni \xi \\ X \subset T}} \Phi_{0}^{(1)}(X) \leq \psi(\beta)\left(\sum_{p=1}^{\infty} p 2^{p} R_{0}^{-p}\right) \leq \psi(\beta) .
$$

Assim, a partir de (II.16), (II.17), (II.18) e (II.19) podemos considerar:

$$
\hat{p}_{N}\left(\mathcal{S}_{X}\right) \propto \exp \left\{-\beta\left|\mathcal{S}_{X}\right|-\sum_{T \subset X} \Phi\left(\mathcal{S}_{T}\right)\right\}=\exp \left\{-\beta\left|\mathcal{S}_{X}\right|-U\left(\mathcal{S}_{X}\right)\right\} .
$$

que viabiliza a interpretação que descrevemos no início dessa seção. Observamos que o lema II.3 informa que os potenciais $\Phi\left(\mathcal{S}_{T}\right)$ são pequenos a baixas temperaturas (como indica (II.22)) e tem curto alcance (por (II.23)); e que $U\left(\mathcal{S}_{X}\right)$ é uma função invariante por translação quando $X$ está longe das bordas.

Além disso, a baixas temperaturas, as atividades dos componentes tornam-se muito pequenas e, portanto podemos esperar que esses componentes do gás de formas tenham um comportamento quase independente um dos outros, de modo a podermos encontrar um resultado análogo ao obtido no capítulo I para o modelo S.O.S. . 


\section{II-5 Expansão em Conglomerados do Gás de Formas}

A maneira pela qual iremos estudar a flutuação de $\lambda$ será através da função característica do salto em um ponto e, a partir da função característica, obter a distribuição de probabilidade do salto, através da fórmula de inversão abaixo:

$$
P\left(\delta \mathcal{S}_{X}=k\right)=P\left(\sum_{\xi \in X} \delta \mathcal{S}_{\xi}=k\right)=\frac{1}{2 \pi} \int_{-\pi}^{\pi} \mathbb{E}\left(e^{i s \delta \mathcal{S}_{X}}\right) e^{-i s k} d s .
$$

Como comentado na seção 2, estamos considerando a distribuição de probabilidade de $\lambda$ sem ter o vínculo inicial de que a linha termina no ponto B. Portanto será necessário fazer um condicionamento posterior.

Consideraremos então a função característica da variável aleatória $\sum_{\xi \in X} \delta \mathcal{S}_{\xi}$ que representa o salto total dado por $\lambda=\mathcal{S}_{X}$. De (II.25) temos que:

$$
\begin{aligned}
\mathbb{E}\left\{e^{i s \sum_{\xi} \delta \mathcal{S}_{\xi}}\right\} & =\frac{\sum_{X \subset[0, N]} \sum_{\mathcal{S}_{X}} \exp \left\{i s \sum_{\xi \in X} \delta \mathcal{S}_{\xi}-\beta\left|\mathcal{S}_{X}\right|-U\left(\mathcal{S}_{X}\right)\right\}}{\sum_{X \subset[0, N]} \sum_{\mathcal{S}_{X}} e^{-\beta\left|\mathcal{S}_{X}\right|-U\left(\mathcal{S}_{X}\right)}} \\
& =\frac{\sum_{X \subset[0, N]} \sum_{\mathcal{S}_{X}} e^{-U\left(\mathcal{S}_{X}\right)} \prod_{\xi \in X} e^{i s \delta \mathcal{S}_{\xi}-\beta\left|\mathcal{S}_{\xi}\right|}}{\sum_{X \subset[0, N]} \sum_{\mathcal{S}_{X}} e^{-U\left(\mathcal{S}_{X}\right)} \prod_{\xi \in X} e^{-\beta\left|\mathcal{S}_{\xi}\right|}} .
\end{aligned}
$$

Para calcular o numerador e o denominador de (II.26) iremos fazer uma nova expansão em conglomerados, só que agora estamos lidando com um gás de partículas de formas com sua respectiva energia potencial $U\left(\mathcal{S}_{X}\right)$.

A expansão do gás de formas é análoga àquela feita na seção 2 porém o espaço de funções aqui é o espaço $\mathcal{F}$ de funções simétricas definidas em conjuntos finitos de configuraçôes ordenadas de partículas de formas obtidas considerando também as configurações com número de ocupação maior que um, ou seja,

$$
\mathcal{F}=\left\{\varphi \text { tal que } \varphi: \mathcal{N} \rightarrow \mathbb{C} \text { é simétrica; }|\varphi|_{n}=\sup _{\substack{\xi_{1}, \ldots, \xi_{n} \\ \mathcal{S}_{\xi_{1}, \ldots, \mathcal{S}_{\xi_{n}}}}}\left|\varphi\left(\mathcal{S}_{\xi_{1}}, \ldots, \mathcal{S}_{\xi_{n}}\right)\right|<\infty, \forall n \geq 0\right\}
$$


onde $\mathcal{N}=\left\{\mathcal{S}_{X} ; \mathcal{S}_{X}=\left(\mathcal{S}_{\xi_{1}}, \ldots, \mathcal{S}_{\xi_{n}}\right)\right.$, cada $\mathcal{S}_{\xi_{i}}$ é uma forma sobre um aglomerado $\xi_{i} \subset$ $\mathbb{Z}\}$, ou seja, $\mathcal{S}_{X}$ é um conjunto finito de partículas de formas, localizadas em $\xi_{1}, \ldots, \xi_{n}$ e sem a restrição de que o número de ocupação seja no máximo 1, de maneira a ser possível termos uma configuração na qual $\xi_{i}=\xi_{j}$ para algum $i \neq j$ e $\mathcal{S}_{\xi_{i}}=\mathcal{S}_{\xi_{j}}$; e $\mathbb{C}$ é o conjunto dos números complexos.

Analogamente às definiçôes II.1 a. II.5 temos:

Definição II.8 Sejam $\mathcal{F}_{0}$ e $\mathcal{F}_{1}$, subespaços de $\mathcal{F}$, onde $\mathcal{F}_{0}=\{\varphi \in \mathcal{F} \mid \varphi(\emptyset)=0\}$ e $\mathcal{F}_{1}=\{\varphi \in \mathcal{F} \mid \varphi(\emptyset)=1\}$, entâo para $\mathcal{S}_{X} \in \mathcal{N}$ definimos:

- $O$ produto de convolução $\varphi_{1} * \varphi_{2} \in \mathcal{F}$ de duas funçốes $\varphi_{1}$ e $\varphi_{2} \in \mathcal{F}$ :

$$
\left(\varphi_{1} * \varphi_{2}\right)\left(\mathcal{S}_{X}\right)=\sum_{X_{1} \cup X_{2}=X} \varphi_{1}\left(\mathcal{S}_{X_{1}}\right) \varphi_{2}\left(\mathcal{S}_{X_{2}}\right),
$$

onde $X$ é um conjunto geral de aglomerados e está determinado pelos diferentes aglomerados em $X$ e suas multiplicidades, e $\sum_{X_{1} \cup X_{2}=X}$ é considerada como uma soma. de pares ordenados $X_{1}, X_{2}$ que decompõem $X$ em dois conjuntos de aglomerados ordenados.

- O elemento neutro da multiplicação de $\mathcal{F}$, i.e., a identidade para o produto (II.27)

$$
\mathbb{1}\left(\mathcal{S}_{X}\right)= \begin{cases}1 & \text { se } X=\emptyset \\ 0 & \text { se } X \neq \emptyset\end{cases}
$$

- A exponencial de uma função $\varphi \in \mathcal{F}_{0}$ :

$$
(\operatorname{Exp} \varphi)\left(\mathcal{S}_{X}\right)=\sum_{n \geq 0} \frac{\varphi^{n}\left(\mathcal{S}_{X}\right)}{n !}=11\left(\mathcal{S}_{X}\right)+\sum_{n \geq 1} \frac{1}{n !} \sum_{X_{1} \cup \ldots \cup X_{n}=X} \varphi\left(\mathcal{S}_{X_{1}}\right) \ldots \varphi\left(\mathcal{S}_{X_{n}}\right) .
$$

- A função inversa da exponencial, o logaritmo, de uma função $\varphi_{1} \in \mathcal{F}_{1}$ com $\varphi_{1}=$ $11+\varphi, \varphi \in \mathcal{F}_{0}$.

$$
\begin{aligned}
\left(\log \varphi_{1}\right)\left(\mathcal{S}_{X}\right) & =(\log (11+\varphi))\left(\mathcal{S}_{X}\right)=\sum_{n \geq 1} \frac{(-1)^{n+1}}{n} \varphi^{n}\left(\mathcal{S}_{X}\right) \\
& =\sum_{n \geq 1} \frac{(-1)^{n+1}}{n} \sum_{X_{1} \cup \ldots \cup X_{n}=X} \varphi\left(\mathcal{S}_{X_{1}}\right) \ldots \varphi\left(\mathcal{S}_{X_{n}}\right) .
\end{aligned}
$$

Observe também que $\operatorname{Exp} \log \varphi_{1}=\varphi_{1}$. 
- Um produto escalar entre elementos de $\mathcal{F}$ e funçôes multiplicativas $\chi_{\theta}, \theta \subset \mathbb{Z}$, i.e., $\chi_{\theta}\left(\mathcal{S}_{X}\right)=\chi_{\theta}\left(\mathcal{S}_{\xi_{1}}\right) \chi_{\theta}\left(\mathcal{S}_{\xi_{2}}\right) \ldots \chi_{\theta}\left(\mathcal{S}_{\xi_{n}}\right)$. Assim, seja $\mathcal{D}_{\theta}=\{\varphi \in \mathcal{F}$ tal que $\left.\sum_{X, \mathcal{S}_{X}}\left|\varphi\left(\mathcal{S}_{X}\right) \chi_{\theta}\left(\mathcal{S}_{X}\right)\right|<\infty\right\}$ e então, se $\varphi \in \mathcal{D}_{\theta}$ o produto escalar é dado por $\left\langle\chi_{\theta}, \varphi\right\rangle=\sum_{X, \mathcal{S}_{X}} \varphi\left(\mathcal{S}_{X}\right) \chi_{\theta}\left(\mathcal{S}_{X}\right)$, onde a soma em $\left(X, \mathcal{S}_{X}\right)$ tem o seguinte significado:

$$
\sum_{X, \mathcal{S}_{X}}(\cdot)=\sum_{n=0}^{\infty} \frac{1}{n !} \sum_{\xi_{1}} \ldots \sum_{\xi_{n}} \sum_{\mathcal{S}_{\xi_{1}}} \ldots \sum_{\mathcal{S}_{\xi_{n}}}(\cdot)
$$

- Uma aplicação $D_{\mathcal{S}_{X}}: \mathcal{F} \rightarrow \mathcal{F}$ dada por $\left(D_{\mathcal{S}_{X}} \varphi\right)\left(\mathcal{S}_{Y}\right)=\varphi\left(\mathcal{S}_{X} \cup \mathcal{S}_{Y}\right)$, com as seguintes propriedades:

$-D_{\mathcal{S}_{\xi}}\left(\varphi_{1} * \varphi_{2}\right)=\left(D_{\mathcal{S}_{\xi}} \varphi_{2}\right) * \varphi_{1}+\varphi_{2} *\left(D_{\mathcal{S}_{\xi}} \varphi_{1}\right)$

$-D_{\mathcal{S}_{\xi}}(\operatorname{Exp} \varphi)=\left(D_{\mathcal{S}_{\xi}} \varphi\right) *(\operatorname{Exp} \varphi)$,

- $D_{\mathcal{S}_{\xi}}(\log \varphi)=\varphi^{-1} *\left(D_{\mathcal{S}_{\xi}} \varphi\right), \varphi \in \mathcal{F}_{1}$ e $\varphi^{-1}$ é a função inversa em relação ao produto de convolução em (II.27), isto é, $\varphi^{-1} * \varphi=\varphi * \varphi^{-1}=11$.

As provas das propriedades descritas acima são análogas as da seção 2, levando-se em conta que o espaço de funções é diferente, e portanto nâo serão feitas.

Com base nessas definiçôes obtemos o lema abaixo:

Lema II.4 Para $\chi_{\theta}$ multiplicativa $e, \varphi \in \mathcal{F}_{0} \quad e \quad \varphi \in \mathcal{D}_{\theta}$, então $\left\langle\chi_{\theta}\right.$, Exp $\left.\varphi\right\rangle=$ $\exp \left\{\left\langle\chi_{\theta}, \varphi\right\rangle\right\}$ ou de maneira mais explícita,

$$
\sum_{X, \mathcal{S}_{X}}(\operatorname{Exp} \varphi)\left(\mathcal{S}_{X}\right) \chi_{\theta}\left(\mathcal{S}_{X}\right)=\exp \left\{\sum_{X, \mathcal{S}_{X}} \varphi\left(\mathcal{S}_{X}\right) \chi_{\theta}\left(\mathcal{S}_{X}\right)\right\} .
$$

A prova desse lema, sendo similar à do lema II.1, também não será feita.

Analogamente ao que foi feito na seção 2, consideraremos funções $\chi_{\theta}$ particulares de modo que o lema II.4 possa ser aplicado para podermos calcular funções características de variáveis nas quais temos interesse. 
Definição II.9 Para $X=\left(\xi_{1}, \ldots, \xi_{n}\right)$ e $\xi_{1}<\xi_{2}<\ldots<\xi_{n}$ (no sentido dos aglomerados serem numerados da esquerda para a direita) definimos as seguintes funções:

$$
\begin{aligned}
U_{1}\left(\mathcal{S}_{X}\right) & =\sum_{\substack{T \subset X \\
T \ni \xi_{1}}} \Phi\left(\mathcal{S}_{T}\right) \\
W_{1}\left(\mathcal{S}_{X}, \mathcal{S}_{Y}\right) & =\sum_{\substack{T \subset X \\
T \ni \xi_{1}}} \Phi\left(\mathcal{S}_{T \cup Y}\right) \text { para } Y \neq \emptyset \text { e } Y \cap X=\emptyset \\
I_{1}\left(\mathcal{S}_{X}, \mathcal{S}_{Y}\right) & =\sum_{\substack{\xi_{1} \ni T \subset X \\
\emptyset \neq P \subset Y}} \Phi\left(\mathcal{S}_{T \cup P}\right)=\sum_{\emptyset \neq P \subset Y} W_{1}\left(\mathcal{S}_{X}, \mathcal{S}_{P}\right) \text { para } Y \neq \emptyset \text { e } Y \cap X=\emptyset \\
K_{1}\left(\mathcal{S}_{X}, \mathcal{S}_{Y^{\prime}}\right) & = \begin{cases}\sum_{n \geq 1} \sum_{\substack{\left\{P_{1}, \ldots, P_{n}\right\} \\
\cup P_{i}=Y}} \prod_{j=1}^{n}\left(e^{-W_{1}\left(\mathcal{S}_{X}, \mathcal{S}_{P_{j}}\right)}-1\right) & \text { se } Y \neq \emptyset, \\
& \text { se } Y=\emptyset .\end{cases}
\end{aligned}
$$

onde $\sum_{\substack{\left\{P_{1}, \ldots, P_{n}\right\} \\ \cup P_{i}=Y}}$ é sobre todas as n-uplas de diferentes subconjuntos não vazios de $Y$ tal que a união deles resulte em $Y$, porém não fazemos a restrição que os $P_{i}$ sejam disjuntos, apenas que sejam todos não nulos.

As funções acima foram definidas com a finalidade de facilitar os cálculos, como veremos posteriormente, quando obtivermos uma equação integral para a função definida em (II.31) adiante, como fizemos na seção 2. Porém, a função $\varphi$ que consideraremos aqui é um pouco diferente e um pouco mais complexa de se trabalhar. Vamos expor duas funções nas quais temos um interesse específico.

Considere a função $\bar{\varphi}$, para um $\beta_{0}>0$ fixado suficientemente grande, dada por

$$
\bar{\varphi}\left(\mathcal{S}_{X}\right)=\left\{\begin{array}{cl}
1 & \text { se } X=\emptyset \\
\exp \left\{-\beta_{0}\left|\mathcal{S}_{X}\right|-U\left(\mathcal{S}_{X}\right)\right\} & \text { se } X=\left(\xi_{1}, \ldots, \xi_{n}\right) \text { e } \xi_{i} \cap \xi_{j}=\emptyset, i \neq j \\
0 & \text { nos outros casos. }
\end{array}\right.
$$

Observamos que de (II.19), (II.21) e (II.22) segue que $\left|U\left(\mathcal{S}_{X}\right)\right| \leq\left|\mathcal{S}_{X}\right| \psi(\beta)$ é finito, de modo que $\bar{\varphi} \in \mathcal{F}$, mais especificamente a $\mathcal{F}_{1}$ e, portanto, podemos definir $\bar{\varphi}^{T}=\log \bar{\varphi}_{1}$ $\operatorname{com} \bar{\varphi}_{1}=11+\bar{\varphi}$.

Como comentado no início desta seção, temos interesse em elementos de $\mathcal{F}_{1}$ que nos 
permitam calcular (II.26), ou seja, vamos querer considerar $\varphi \in \mathcal{F}_{1}$ da forma:

$$
\varphi\left(\mathcal{S}_{X}\right)=\left\{\prod_{\xi \in X} \eta\left(\mathcal{S}_{\xi}\right)\right\} \bar{\varphi}\left(\mathcal{S}_{X}\right)
$$

para $\eta\left(\mathcal{S}_{\xi}\right) \in \mathbb{C}$, onde $\mathbb{C}$ é o conjunto dos números complexos, tal que $\left|\eta\left(\mathcal{S}_{\xi}\right)\right| \leq 1$.

De (II.28) temos que

$$
\varphi^{T}\left(\mathcal{S}_{X}\right)=(\log \varphi)\left(\mathcal{S}_{X}\right)=\left\{\prod_{\xi \in X} \eta\left(\mathcal{S}_{\xi}\right)\right\} \bar{\varphi}^{T}\left(\mathcal{S}_{X}\right)
$$

bastando, portanto, analisarmos $\bar{\varphi}^{T}$. Consideraremos para isso, a função $\Delta$ abaixo:

Definição II.10 Para $X \in \mathcal{N}$, um conjunto de aglomerados sem sobreposição, $Y \in \mathcal{N}$ qualquer e $\bar{\varphi}$ dada por (II.30) definimos

$$
\Delta_{\mathcal{S}_{X}}\left(\mathcal{S}_{Y^{\prime}}\right)=\left\{\begin{array}{cl}
11\left(\mathcal{S}_{Y}\right) & \text { se } X=\emptyset \\
\left(\bar{\varphi}^{-1} * D_{\mathcal{S}_{X}} \bar{\varphi}\right)\left(\mathcal{S}_{Y^{\prime}}\right) & \text { se } X \neq \emptyset \text { e só contém aglomerados que } \\
& \text { náo se sobrepóem, } e \\
0 & \text { nos outros casos. }
\end{array}\right.
$$

Com os resultados acima e a partir da expressão (II.31) chegamos ao seguinte lema.

Lema II.5 Para $\mathcal{S}_{Y}$ qualquer e $\mathcal{S}_{X}$ tal que $X$ contém apenas aglomerados que não se sobrepõem, temos:

$$
\Delta_{\mathcal{S}_{\xi \cup X}}\left(\mathcal{S}_{Y^{\prime}}\right)=e^{-\beta_{0}\left|\mathcal{S}_{\xi}\right|-U_{1}\left(\mathcal{S}_{X \cup \xi)}\right)} \sum_{\substack{T \subset Y^{\prime} \\ T \cap\left(\xi \cup X^{\prime}\right)=\emptyset}} K_{1}\left(\mathcal{S}_{X \cup \xi}, \mathcal{S}_{T}\right) \sum_{\substack{P \subset Y \backslash T \\ P \cap \xi \neq \emptyset}}(-1)^{N(P)} \Delta_{\mathcal{S}_{X \cup T \cup P}}\left(\mathcal{S}_{Y \backslash(T \cup P)}\right)
$$

onde $\overline{P \cap \xi} \neq \emptyset$ significa que todos os elementos em $P$ interseptam $\xi$ ou $P=\emptyset$. A prova deste lema se encontra no apêndice B.

Como queremos mostrar que o lema II.4 pode ser aplicado para calcular funções características, temos que provar que $\bar{\varphi}^{T} \in \mathcal{D}_{\theta}$. Entretanto, observemos que

$$
\bar{\varphi}^{T}\left(\mathcal{S}_{\xi \cup Y}\right)=D_{\mathcal{S}_{\xi}} \bar{\varphi}^{T}\left(\mathcal{S}_{Y}\right)=\left(D_{\mathcal{S}_{\xi}} \log \bar{\varphi}\right)\left(\mathcal{S}_{Y}\right)=\left(\bar{\varphi}^{-1} *\left(D_{\mathcal{S}_{\xi}} \bar{\varphi}\right)\right)\left(\mathcal{S}_{Y}\right)=\Delta_{\mathcal{S}_{\xi}}\left(\mathcal{S}_{Y}\right)
$$


Vamos então estudar um pouco mais a função $\Delta$, considerando a seguinte função:

$$
I_{m}=\sup _{\substack{\mathcal{S}_{\xi_{1}}, \ldots, \mathcal{S}_{\xi_{n}} \\ m \geq n \geq 1}} \sum_{\substack{Y \\ N(Y)=m-n}} \sum_{\mathcal{S}_{Y}^{\prime}}\left|\Delta_{\mathcal{S}_{\xi_{1}}, \ldots, \mathcal{S}_{\xi_{n}}}\left(\mathcal{S}_{Y}^{\prime}\right)\right| \exp \left\{\frac{\beta_{0}}{2} \sum_{i=1}^{n}\left|\mathcal{S}_{\xi_{i}}\right|\right\}
$$

A partir do lema II.5 podemos calcular:

$$
\begin{aligned}
& \sum_{\substack{Y \\
N(Y)+N(X)=m}} \sum_{\mathcal{S}_{Y}^{\prime}}\left|\Delta_{\mathcal{S}_{\xi \cup X}}\left(\mathcal{S}_{Y}^{\prime}\right)\right| e^{\frac{\beta_{0}}{2}\left(\left|\mathcal{S}_{\xi}\right|+\left|\mathcal{S}_{X}\right|\right)} \\
& \leq e^{-\frac{\beta_{0}\left|S_{\xi}\right|}{2}-U_{1}\left(\mathcal{S}_{X \cup \xi}\right)} \sum_{\substack{Y \\
N(Y)+N(X)=m}} \sum_{\mathcal{S}_{Y}^{\prime}} \sum_{\substack{T \subset Y \\
T \cap(\xi \cup X)=\emptyset}}\left|K_{1}\left(\mathcal{S}_{X \cup \xi}, \mathcal{S}_{Y}^{\prime}\right)\right| \times \\
& \times \sum_{\frac{P \subset Y \backslash T}{P \cap \xi \notin \emptyset}}\left|\Delta_{\mathcal{S}_{X \cup T \cup P}}\left(\mathcal{S}_{Y \backslash(T \cup P)}^{\prime}\right)\right| e^{\frac{\beta_{0}}{2}\left(\left|\mathcal{S}_{X \cup T \cup P}\right|\right)} e^{-\frac{\beta_{0}}{2}\left(\left|\mathcal{S}_{X \cup T \cup P}\right|\right)} \\
& \leq e^{-\frac{\beta_{0}\left|S_{\xi}\right|}{2}-U_{1}\left(\mathcal{S}_{X \cup \xi}\right)} \sum_{\substack{T: T \cap P=\emptyset \\
(T \cup P) \cap X=\emptyset}} \sum_{\substack{P: P \cap T=\emptyset \\
P \cap \xi \neq \emptyset \\
N(P)+N(T)+N(X) \leq m}} \sum_{\mathcal{S}_{P \cup X}^{\prime}}\left|K_{1}\left(\mathcal{S}_{X \cup \xi}, \mathcal{S}_{T}^{\prime}\right)\right| \times \\
& \times \sum_{\substack{Y: Y \supset T \cup P \\
N(Y \backslash(T \cup P))+N(P)+N(T)+N(X)=m}} \sum_{\mathcal{S}_{Y \backslash(T \cup P)}^{\prime}} \frac{\left|\Delta_{\mathcal{S}_{X \cup T \cup P}}\left(\mathcal{S}_{Y \backslash(T \cup P)}^{\prime}\right)\right|}{e^{-\frac{\beta_{0}}{2}\left|\mathcal{S}_{X \cup T \cup P}\right|}} e^{-\frac{\beta_{0}}{2}\left|\mathcal{S}_{X \cup T \cup P}\right|} \\
& \leq I_{m}\left\{e^{-\frac{\beta_{0}\left|S_{\xi}\right|}{2}-U_{1}\left(\mathcal{S}_{X \cup \xi}\right)} \sum_{\substack{T \\
T \cap X=\emptyset}} \sum_{\substack{P: P \cap T=\emptyset \\
P \cap X=\emptyset \\
P \cap \xi \neq \emptyset}} \sum_{\mathcal{S}_{T \cup P}^{\prime}}\left|K_{1}\left(\mathcal{S}_{X \cup \xi}, \mathcal{S}_{T}^{\prime}\right)\right| e^{-\frac{\beta_{0}\left|S_{T \cup P}^{\prime}\right|}{2}}\right\} \\
& \leq I_{m} \kappa\left(\beta, \beta_{0}\right),
\end{aligned}
$$

onde $\kappa\left(\beta, \beta_{0}\right) \rightarrow 0$ exponencialmente rápido quando $\beta-\beta_{0}$ e $\beta_{0}$ tendem ao infinito. A última passagem (para $\kappa\left(\beta, \beta_{0}\right)$ ) está feita em detalhes no apêndice 4 de [Gal].

Da expressão (II.33) temos que $I_{m+1} \leq I_{m} \kappa\left(\beta, \beta_{0}\right)$, e recursivamente obtemos: $I_{m} \leq I_{1} \kappa\left(\beta, \beta_{0}\right)^{m-1}$. E como $I_{1} \leq e^{-\frac{\beta_{0}}{2}}$ segue que $I_{m+1} \leq \kappa\left(\beta, \beta_{0}\right)^{m-1} e^{-\frac{\beta_{0}}{2}}$.

Assim, obtemos a seguinte estimativa, se $\beta$ é grande o suficiente:

$$
\begin{aligned}
\sum_{Y} \sum_{\mathcal{S}_{Y}}\left|\bar{\varphi}^{T}\left(\mathcal{S}_{\xi \cup Y}\right)\right| & =e^{ \pm \beta_{0}\left|\mathcal{S}_{\xi}\right|} \sum_{m=1}^{\infty} \sum_{\substack{Y \\
N(Y)=m-1}} \sum_{\mathcal{S}_{Y^{\prime}}}\left|\Delta_{\mathcal{S}_{\xi}}\left(\mathcal{S}_{Y}\right)\right| \leq e^{-\beta_{0}\left|\mathcal{S}_{\xi}\right|} \sum_{m=1}^{\infty} I_{m} \\
& \leq e^{-\frac{\beta_{0}}{2}\left|\mathcal{S}_{\xi}\right|} \sum_{m \geq 1} \kappa\left(\beta, \beta_{0}\right)^{m-1} e^{-\frac{\beta_{0}}{2}}=\frac{e^{-\frac{\beta_{0}}{2}\left(\left|\mathcal{S}_{\xi}\right|+1\right)}}{1-\kappa\left(\beta, \beta_{0}\right)}
\end{aligned}
$$


Uma conseqüência simples de (II.34) é que para $\beta$ e $\beta_{0}\left(\beta>\beta_{0}\right)$ suficientemente grande temos $\sum_{X \subset[0, N]} \sum_{\mathcal{S}_{X}}\left|\bar{\varphi}^{T}\left(\mathcal{S}_{X}\right)\right|<\infty$, o que implica que podemos utilizar o lema II.4 para

$$
\chi_{\theta}\left(\mathcal{S}_{X}\right)= \begin{cases}1 & \text { se } X \subset \theta \\ 0 & \text { se } X \not \subset \theta\end{cases}
$$

$\operatorname{com} \theta=[0, N]$.

\section{II-6 Teorema Limite Central para os saltos sobre intervalos}

Nesta seção iremos mostrar a convergência das distribuições finito-dimensionais dos saltos sobre intervalos para as da ponte Browniana.

Consideremos um inteiro $k \geq 1$ e $0=t_{0}<t_{1}<\ldots<t_{k+1}=1$ e definimos $a_{j} \equiv a_{j}^{(N)}=\left\lfloor N t_{j}\right\rfloor, j=0,1, \ldots, k+1$ e as funções indicadoras $\chi_{j}$ para $j=0,1, \ldots, k+1$ :

$$
\begin{aligned}
\chi_{0}(\cdot) \equiv \chi_{0}^{(N)}(\cdot) & =0 \text { e } \\
\chi_{j}\left(\mathcal{S}_{\xi}\right) \equiv \chi_{j}^{(N)}\left(\mathcal{S}_{\xi}\right) & = \begin{cases}1 & \text { se } \xi \subset\left[0, a_{j}\right], \\
0 & \text { caso contrário. }\end{cases}
\end{aligned}
$$

Então para cada $j, \sum_{\xi \in X} \chi_{j}\left(\mathcal{S}_{\xi}\right) \delta \mathcal{S}_{\xi}$ representa o salto total de $\mathcal{S}_{X}$ entre 0 e $a_{j}$, e $\delta \mathcal{S}_{X}=\sum_{\xi \in X} \delta \mathcal{S}_{\xi}$, como na definição II.7.

Abaixo apresentamos o teorema que estabelece a convergência das distribuições finito-dimensionais dos saltos para a ponte Browniana.

Teorema II.1 Para $\beta>\beta_{0}>0$ suficientemente grandes e para reais $T_{j}<T_{j}^{\prime}$, $j=1, \ldots, k$ :

$$
\begin{aligned}
P\left(T_{j} \sigma \sqrt{N} \leq \sum_{\xi \in X} \chi_{j}\left(\mathcal{S}_{\xi}\right) \delta \mathcal{S}_{\xi}\right. & \left.\leq T_{j}^{\prime} \sigma \sqrt{N}, j=1, \ldots, k \mid \delta \mathcal{S}_{X}=0\right) \\
& \stackrel{N \rightarrow \infty}{\longrightarrow} P^{*}\left(\mathcal{B}_{t_{j}} \in\left[T_{j}, T_{j}^{\prime}\right], j=1, \ldots, k\right)
\end{aligned}
$$


onde $\sigma=\sigma\left(\beta, \beta_{0}\right)>0$ é uma constante e $\left(\mathcal{B}_{t}, P^{*}\right)$ é uma ponte Browniana unidimensional. A forma explícita de $P^{*}$ é dada pela expressão (A.1) no apêndice $A$.

Prova: A prova é feita a partir da fórmula de inversão e está dividida em etapas que são dadas pelos resultados que apresentamos abaixo em forma de lemas e uma proposição.

Utilizando os resultados da seção 5, vamos calcular a função característica do vetor aleatório $k$-dimensional onde o $j$-ésimo termo do vetor representa o salto total efetuado por $\mathcal{S}_{X}$ entre 0 e o $j$-ésimo intervalo.

Para $\tilde{s}_{1}, \tilde{s}_{2}, \ldots, \tilde{s}_{k+1} \in \mathbb{R}$, definimos a função $f_{N}=f_{N}\left(\tilde{s}_{1}, \tilde{s}_{2}, \ldots, \tilde{s}_{k+1}\right): \mathcal{N} \longrightarrow \mathbb{R}$ por:

$$
f_{N}\left(\mathcal{S}_{X}\right)=\sum_{j=1}^{k+1} \tilde{s}_{j} \sum_{\xi \in X} \chi_{j}\left(\mathcal{S}_{\xi}\right) \delta \mathcal{S}_{\xi} .
$$

Então de (II.25) temos que $\mathbb{E}\left\{e^{i f_{N}\left(\mathcal{S}_{X}\right)}\right\}=\sum_{X \subset[0, N]} \sum_{\mathcal{S}_{X}} e^{i f_{N}\left(\mathcal{S}_{X}\right)} \hat{p}_{N}\left(\mathcal{S}_{X}\right)$ é a função característica do vetor aleatório $\left(\sum_{\xi \in X} \chi_{j}\left(\mathcal{S}_{\xi}\right) \delta \mathcal{S}_{\xi}, j=1, \ldots, k+1\right)$ que representa os saltos sobre intervalos.

A distribuição $k$-dimensional dos saltos para $m_{0}=0$ e $m_{1}, m_{2}, \ldots, m_{k+1} \in \mathbb{Z}$ é dada, através da fórmula de inversão, por:

$$
\begin{aligned}
& P\left(\sum_{\xi \in X} \chi_{j}\left(\mathcal{S}_{\xi}\right) \delta \mathcal{S}_{\xi}=m_{j}, j=1,2, \ldots, k+1\right)= \\
& \left(\frac{1}{2 \pi}\right)^{k+1} \int_{-\pi}^{\pi} \cdots \int_{-\pi}^{\pi} \mathbb{E}\left\{e^{i f_{N}\left(s_{X}\right)}\right\} \exp \left\{-i \sum_{j=1}^{k+1} \tilde{s}_{j} m_{j}\right\} d \tilde{s}_{1} \ldots d \tilde{s}_{k+1}= \\
& \left(\frac{1}{2 \pi \sigma \sqrt{N}}\right)^{k+1} \int_{-\pi \sigma \sqrt{N}}^{\pi \sigma \sqrt{N}} \cdots \int_{-\pi \sigma \sqrt{N}}^{\pi \sigma \sqrt{N}} \mathbb{E}\left\{e^{i \frac{\mathcal{s}_{N}\left(s_{X}\right)}{\sigma \sqrt{N}}}\right\} \exp \left\{-i \sum_{j=1}^{k+1} \frac{\tilde{s}_{j} m_{j}}{\sigma \sqrt{N}}\right\} d \tilde{s}_{1} \ldots d \tilde{s}_{k+1} .
\end{aligned}
$$

Para calcular a função característica, usamos o lema II.4:

$$
\mathbb{E}\left\{e^{i \frac{f_{N}\left(s_{X}\right)}{\sigma \sqrt{N}}}\right\}=\frac{\sum_{X \subset[0, N]} \sum_{\mathcal{S}_{X}} e^{-U\left(\mathcal{S}_{X}\right)+i \frac{\mathcal{f}_{N}\left(s_{X}\right)}{\sigma \sqrt{N}}-\beta\left|\mathcal{S}_{X}\right|}}{\sum_{X \subset[0, N]} \sum_{\mathcal{S}_{X}} e^{-U\left(\mathcal{S}_{X}\right)-\beta\left|\mathcal{S}_{X}\right|}}
$$




$$
\begin{aligned}
& =\frac{\sum_{X \subset[0, N]} \sum_{\mathcal{S}_{X}} \bar{\varphi}\left(\mathcal{S}_{X}\right) e^{-\left(\beta-\beta_{0}\right)\left|\mathcal{S}_{X}\right|} e^{i \frac{\mathcal{J}_{N}\left(\mathcal{S}_{X}\right)}{\sigma \sqrt{N}}}}{\sum_{X \subset[0, N]} \sum_{\mathcal{S}_{X}} \bar{\varphi}\left(\mathcal{S}_{X}\right) e^{-\left(\beta-\beta_{0}\right)\left|\mathcal{S}_{X}\right|}} \\
& =\exp \left\{\sum_{X \subset[0, N]} \sum_{\mathcal{S}_{X}} \bar{\varphi}^{T}\left(\mathcal{S}_{X}\right) e^{-\left(\beta-\beta_{0}\right)\left|\mathcal{S}_{X}\right|}\left(e^{i \frac{\mathcal{S}_{N}\left(\mathcal{S}_{X}\right)}{\sigma \sqrt{N}}}-1\right)\right\} .
\end{aligned}
$$

Podemos fazer uma transformação de variáveis de maneira a tornar $f_{N}$ adequada a algumas manipulações que nos facilitem o seu cálculo. Com esse fim, consideremos $g_{j}\left(\mathcal{S}_{\xi}\right)=g_{j}^{(N)}\left(\mathcal{S}_{\xi}\right) \equiv \chi_{j}\left(\mathcal{S}_{\xi}\right)-\chi_{j-1}\left(\mathcal{S}_{\xi}\right)$ e $s_{j}=\tilde{s}_{j}+\tilde{s}_{j+1}+\ldots+\tilde{s}_{k+1}$ para $j=1,2, \ldots, k+1$. Portanto,

$$
f_{N}\left(\mathcal{S}_{X}\right)=\sum_{j=1}^{k+1} s_{j} \sum_{\xi \in X} g_{j}\left(\mathcal{S}_{\xi}\right) \delta \mathcal{S}_{\xi}
$$

Observamos que $g_{j}\left(\mathcal{S}_{\xi}\right) \neq \mathbb{1}_{\left[a_{j-1}+1, a_{j}\right]}(\xi)$. Na realidade gostaríamos que a igualdade valesse, porque teríamos a independência dos saltos em intervalos disjuntos. Com a independência a função característica dos saltos seria o produto da função característica do salto em cada intervalo. A proposição abaixo nos fornece esse resultado no limite termodinâmico.

Proposição II.2 Quando $N \rightarrow \infty$,

$$
\mathbb{E}\left\{e^{i \frac{f_{N}\left(S_{X}\right)}{\sigma \sqrt{N}}}\right\}=\exp \left\{\sum_{j=1}^{k+1} \sum_{X \subset\left[a_{j-1}+1, a_{j}\right]} \sum_{\mathcal{S}_{X}} \bar{\varphi}^{T}\left(\mathcal{S}_{X}\right) e^{-\left(\beta-\beta_{0}\right)\left|\mathcal{S}_{X}\right|}\left(e^{i s_{j} \frac{\delta S_{X}}{\sigma \sqrt{N}}}-1\right)+o(N)\right\}
$$

A prova dessa proposição, que está feita no apêndice B, assim como outros resultados que veremos adiante seguem do lema abaixo, cuja prova também está feita no apêndice B.

Lema II.6 $\quad \lim _{N \rightarrow \infty} \sup _{p \in[0, N]} \frac{1}{N} \sum_{\substack{X \subset[0, N] \\ X \ni p}} \sum_{\mathcal{S}_{X}}\left|\bar{\varphi}^{T}\left(\mathcal{S}_{X}\right)\right|=0$.

Como a função $\mathbb{E}\left\{e^{i \frac{f_{N}\left(s_{X}\right)}{\sigma \sqrt{N}}}\right\}$ é uma função periódica de período $2 \pi \sigma \sqrt{N}$ para cada $s_{j}$, basta que consideremos $\left|s_{j}\right| \leq \pi \sigma \sqrt{N}$ para $j=1,2, \ldots, k+1$. Analisando a função característica dependendo da variação dos $s_{j}$ 's temos que a região relevante se restringe 
a $\left|s_{j}\right| \leq \log N$. Esse resultado é estabelecido pelo lema a seguir. Como no sistema inicial temos a interface presa nas bordas, vamos calcular a distribuição em (II.35) com $m_{k+1}=0$ que resulta em $\delta \mathcal{S}_{X}=0$.

Lema II.7 Para $\beta>\beta_{0}>0$ suficientemente grande e dados $m_{1}, m_{2}, \ldots, m_{k} \in \mathbb{Z}$, com $m_{0}=m_{k+1}=0$ :

$$
\begin{aligned}
& P\left(\sum_{\xi \in X} \chi_{j}\left(\mathcal{S}_{\xi}\right) \delta \mathcal{S}_{\xi}=m_{j}, j=1,2, \ldots, k, \delta \mathcal{S}_{X}=0\right) \\
& \quad=\left(\frac{1}{2 \pi \sigma \sqrt{N}}\right)^{k+1} \int_{\left|s_{j}\right| \leq \log N, \forall j} \ldots \int_{N}\left\{\mathbb{E}\left\{e^{i \frac{f_{N}\left(s_{X}\right)}{\sigma \sqrt{N}}}\right\} \prod_{j=1}^{k+1} e^{-i s_{j} \frac{\left(m_{j}-m_{j-1}\right)}{\sigma \sqrt{N}}}\right\} d s_{1} \ldots d s_{k+1}+o\left(\frac{1}{N^{l}}\right),
\end{aligned}
$$

quando $N \rightarrow \infty$, para algum $l$ inteiro e positivo. E dado $\varepsilon, 0<\varepsilon<1$, existe $\beta(\varepsilon)>0$ tal que se $\beta-\beta_{0}>\beta(\varepsilon)$, então para os $j$ 's $(1 \leq j \leq k+1)$ tais que $\varepsilon \sigma \sqrt{N} \leq\left|s_{j}\right| \leq \pi \sigma \sqrt{N}$ temos que $\mathbb{E}\left\{e^{i \frac{f_{N}\left(s_{X}\right)}{\sigma \sqrt{N}}}\right\} \leq e^{-c N}, c>0$.

Prova do lema II.7: Vamos analisar primeiro a contribuição de um $X=\{\xi\}$ com $|\xi|=0$ e $\left|\mathcal{S}_{\xi}\right|=1$ (em conseqüência $\left|\delta \mathcal{S}_{\xi}\right|=1$ ), sendo $\xi \neq 0, N$. Antes observemos que de (II.32), lema II.5, definição II.9 e lema II.3 decorre que

$$
\bar{\varphi}^{T}\left(\mathcal{S}_{\xi}\right)=e^{-\beta_{0}\left|\mathcal{S}_{\xi}\right|-U_{1}\left(\mathcal{S}_{\xi}\right)}=e^{-\beta_{0}\left|\mathcal{S}_{\xi}\right|-\Phi\left(\mathcal{S}_{\xi}\right)} \leq e^{-\beta_{0}\left|\mathcal{S}_{\xi}\right|-\psi(\beta)\left|\mathcal{S}_{\xi}\right|}
$$

Isso nos fornece:

$\sum_{X \subset\left[a_{j-1}+1, a_{j}\right]} \sum_{\mathcal{S}_{X}} \bar{\varphi}^{T}\left(\mathcal{S}_{X}\right) e^{-\left(\beta-\beta_{0}\right)\left|\mathcal{S}_{X}\right|}\left(e^{i \frac{s_{j} \delta S_{X}}{\sigma \sqrt{N}}}-1\right)$

$$
\begin{aligned}
\leq & \sum_{\substack{\xi \subset\left[a_{j}-1+1, a_{j}\right] \\
|\xi|=0}} \sum_{\left|\mathcal{S}_{\xi}\right|=1} e^{-\beta_{0}\left|\mathcal{S}_{\xi}\right|-\psi(\beta)\left|\mathcal{S}_{\xi}\right|} e^{-\left(\beta-\beta_{0}\right)\left|\mathcal{S}_{\xi}\right|}\left(e^{i \frac{s_{j} \delta S_{\xi}}{\sigma \sqrt{N}}}-1\right) \\
& +2 \sum_{X \subset\left[a_{j-1}+1, a_{j}\right]\left|\mathcal{S}_{X}\right| \geq 2}\left|\bar{\varphi}^{T}\left(\mathcal{S}_{X}\right)\right| e^{-\left(\beta-\beta_{0}\right)\left|\mathcal{S}_{X}\right|} \\
\leq & N\left(t_{j}-t_{j-1}\right) e^{-\beta-\psi(\beta)}\left(e^{i \frac{s_{j}}{\sigma \sqrt{N}}}-1+e^{-i \frac{s_{j}}{\sigma \sqrt{N}}}-1\right) \\
& +2 e^{-2\left(\beta-\beta_{0}\right)}\left(t_{j}-t_{j-1}\right) \sum_{X \subset[0, N]\left|\mathcal{S}_{X}\right| \geq 2}\left|\bar{\varphi}^{T}\left(\mathcal{S}_{X}\right)\right| .
\end{aligned}
$$


Considerando o fato que existe no máximo $3^{\left|\mathcal{S}_{\xi}\right|+|\xi|} \leq 3^{2}\left|\mathcal{S}_{\xi}\right|$ diferentes formas sobre um aglomerado $\xi$ com um mesmo comprimento $\left|\mathcal{S}_{\xi}\right|$ e a estimativa obtida em (II.34) temos que:

$$
\begin{gathered}
\sum_{X \subset[0, N]} \sum_{\left|\mathcal{S}_{X}\right| \geq 2}\left|\bar{\varphi}^{T}\left(\mathcal{S}_{X}\right)\right| \leq \sum_{\xi \subset[0, N]} \sum_{\mathcal{S}_{\xi}} \sum_{Y, \mathcal{S}_{Y}}\left|\bar{\varphi}^{T}\left(\mathcal{S}_{\xi \cup Y}\right)\right| \leq \sum_{\xi \subset[0, N]} \sum_{\mathcal{S}_{\xi}} \frac{e^{-\frac{\beta_{0}}{2}\left(\left|\mathcal{S}_{\xi}\right|+1\right)}}{1-\kappa\left(\beta, \beta_{0}\right)} \\
\leq \frac{e^{-\frac{\beta_{0}}{2}}}{1-\kappa\left(\beta, \beta_{0}\right)} \sum_{\xi \subset[0, N]} \sum_{p=|\xi|+1}^{\infty}\left(3^{2} e^{-\frac{\beta_{0}}{2}}\right)^{p} \leq N C\left(\beta, \beta_{0}\right),
\end{gathered}
$$

onde $C\left(\beta, \beta_{0}\right) \rightarrow 0$ se $\beta_{0}$ e $\beta-\beta_{0}$ tendem ao infinito.

Com respeito à primeira soma do lado direito de (II.37) lembramos que $\left(e^{i r}-1+e^{-i r}-1\right)=-4 \operatorname{sen}^{2}\left(\frac{r}{2}\right)$. Também, para $0<\varepsilon<1$, se $\varepsilon \sigma \sqrt{N} \leq\left|s_{j}\right| \leq \pi \sigma \sqrt{N}$, então $-4 \operatorname{sen}^{2}\left(\frac{s_{j}}{2 \sigma \sqrt{N}}\right) \leq-4 \operatorname{sen}^{2} \frac{\varepsilon}{2}$.

Portanto usando o fato acima e (II.38) obtemos:

$\sum_{X \subset\left[a_{j-1}+1, a_{j}\right]} \sum_{\mathcal{S}_{X}} \bar{\varphi}^{T}\left(\mathcal{S}_{X}\right) e^{-\left(\beta-\beta_{0}\right)\left|\mathcal{S}_{X}\right|}\left(e^{i \frac{s_{j} \delta S_{X}}{\sigma \sqrt{N}}}-1\right)$

$$
\begin{aligned}
& \leq N\left(t_{j}-t_{j-1}\right) e^{-\beta-\psi(\beta)}\left\{-4 \operatorname{sen}^{2} \frac{\varepsilon}{2}+2 e^{-2 \beta_{0}-\beta+\psi(\beta)} C\left(\beta, \beta_{0}\right)\right\} \\
& \leq N\left(t_{j}-t_{j-1}\right) e^{-\beta-\psi(\beta)}\left\{-2 \operatorname{sen}^{2} \frac{\varepsilon}{2}\right\},
\end{aligned}
$$

onde última desigualdade é válida se tomarmos $\beta_{0}$ e $\beta-\beta_{0}$ suficientemente grandes tal que $2 \operatorname{sen}^{2} \frac{\varepsilon}{2}>2 e^{-2 \beta_{0}-\beta+\psi(\beta)} C\left(\beta, \beta_{0}\right)$.

Então para $\varepsilon \sigma \sqrt{N} \leq\left|s_{j}\right| \leq \pi \sigma \sqrt{N}$ e $\beta_{0}, \beta-\beta_{0}$ suficientemente grandes temos, por (II.39) e pela proposição II.2, que

$$
\begin{aligned}
\mathbb{E}\left\{e^{i \frac{f_{N}\left(s_{X}\right)}{\sigma \sqrt{N}}}\right\} & \leq \exp \left\{\sum_{j=1}^{k+1} N\left[\left(t_{j}-t_{j-1}\right) e^{-\beta-\psi(\beta)}\left(-2 \operatorname{sen}^{2} \frac{\varepsilon}{2}\right)+\frac{o(N)}{N}\right]\right\} \\
& \leq e^{-c N}
\end{aligned}
$$

para alguma constante $c$ positiva.

Para outros $j$ 's tais que $\left|s_{j}\right| \leq \varepsilon \sigma \sqrt{N}$ temos que obter outra estimativa. Expandindo em série de Taylor $\left(e^{i s \frac{\delta S_{X}}{\sigma \sqrt{N}}}-1\right)$ e, observando que em distribuições de probabilidades simétricas em torno do zero a funçâo característica é real, de modo que a parte 
complexa se anula, então a contribuição para a função característica é dada por

$$
\begin{aligned}
& \sum_{X \subset\left[a_{j-1}+1, a_{j}\right]} \sum_{\mathcal{S}_{X}} \bar{\varphi}^{T}\left(\mathcal{S}_{X}\right) e^{-\left(\beta-\beta_{0}\right)\left|\mathcal{S}_{X}\right|}\left(e^{i s_{j} \frac{\delta S_{X}}{\sigma \sqrt{N}}}-1\right)= \\
& \sum_{X \subset\left[a_{j-1}+1, a_{j}\right]} \sum_{\mathcal{S}_{X}} \bar{\varphi}^{T}\left(\mathcal{S}_{X}\right) e^{-\left(\beta-\beta_{0}\right)\left|\mathcal{S}_{X}\right|}\left(-\frac{s_{j}^{2}}{2} \frac{\left(\delta \mathcal{S}_{X}\right)^{2}}{\sigma^{2} N}+\frac{s_{j}^{4}}{24} \frac{\left(\delta \mathcal{S}_{X}\right)^{4}}{\sigma^{4} N^{2}} \mathcal{I}\left(s_{j}, \delta \mathcal{S}_{X}\right)\right),
\end{aligned}
$$

onde $|\mathcal{I}| \leq 1$.

Consideremos agora os seguintes limites, cuja existência está garantida em [Gal].

$$
\begin{aligned}
& \sigma^{2}(\beta) \stackrel{\text { def }}{=} \lim _{N \rightarrow \infty} \frac{1}{N} \sum_{X \subset[0, N]} \sum_{\mathcal{S}_{X}} \bar{\varphi}^{T}\left(\mathcal{S}_{X}\right) e^{-\left(\beta-\beta_{0}\right)\left|\mathcal{S}_{X}\right|}\left(\delta \mathcal{S}_{X}\right)^{2} \\
& \text { e } \quad E_{4}(\beta) \stackrel{\text { def }}{=} \lim _{N \rightarrow \infty} \frac{1}{N} \sum_{X \subset[0, N]} \sum_{\mathcal{S}_{X}}\left|\bar{\varphi}^{T}\left(\mathcal{S}_{X}\right)\right| e^{-\left(\beta-\beta_{0}\right)\left|\mathcal{S}_{X}\right|}\left(\delta \mathcal{S}_{X}\right)^{4} .
\end{aligned}
$$

sendo que $E_{4} \leq 2 \sigma^{2}$ como consta em [Hig].

Em consequência da proposição II.2 e das expressões (II.41) a (II.43), se $\left|s_{j}\right| \leq \varepsilon \sigma \sqrt{N}$ com $\varepsilon<1$, decorre que

$$
\begin{aligned}
\mathbb{E}\left\{e^{\left.i \frac{f_{N}\left(s_{X}\right)}{\sigma \sqrt{N}}\right\}}\right. & =\exp \left\{\sum_{j=1}^{k+1}-\frac{s_{j}^{2}}{2 \sigma^{2}}\left[\left(t_{j}-t_{j-1}\right)\left(\sigma^{2}-\frac{s_{j}^{2}}{12 \sigma^{2}} \frac{E_{4}}{N}\right)+o(1)\right]\right\} \\
& \leq \exp \left\{\sum_{j=1}^{k+1}-\frac{s_{j}^{2}}{2 \sigma^{2}}\left[\left(t_{j}-t_{j-1}\right)\left(\sigma^{2}-\frac{\sigma^{2}}{6}\right)+o(1)\right]\right\} \\
& \leq \exp \left\{\sum_{j=1}^{k+1}-\frac{s_{j}^{2}}{3 \sigma^{2}}\left(t_{j}-t_{j-1}+o(1)\right)\right\}
\end{aligned}
$$

resultando que:

$$
\begin{aligned}
& \left|\int_{\substack{\left|s_{j}\right| \leq \varepsilon \sigma \sqrt{N}, \forall j \\
\left|s_{1}\right| \geq \log N}}\left\{\mathbb{E}\left\{e^{i \frac{f_{N}\left(s_{\cdot}\right)}{\sigma \sqrt{N}}}\right\} \prod_{j=1}^{k+1} e^{-i s_{j} \frac{\left(m_{j}-m_{j-1}\right)}{\sigma \sqrt{N}}}\right\} d s_{1} \ldots d s_{k+1}\right| \\
& \leq 2 \int_{-\infty}^{\infty} \cdots \int_{-\infty}^{\infty} \prod_{j=2}^{k+1} e^{-\frac{s_{j}^{2}}{3}\left(t_{j}-t_{j-1}\right)} d s_{2} \ldots d s_{k+1} \int_{\log N}^{\infty} e^{-\frac{s^{2} t_{1}}{3}} d s_{1} \\
& =2 \pi^{k+1} \prod_{j=2}^{k+1} \sqrt{\frac{t_{j}-t_{j-1}}{3}} \int_{\log N}^{\infty} e^{-\frac{s_{1}^{2} t_{1}}{3}} d s_{1} \\
& \leq \frac{c_{1}}{\log N} e^{-c_{2}(\log N)^{2}}
\end{aligned}
$$


para $c_{1}$ e $c_{2}$ constantes positivas.

Então de (II.40) e (II.44) segue o lema.

O próximo lema nos fornece uma estimativa adequada para $\left|s_{j}\right| \leq \log N$, $j=1, \ldots, k+1$ :

Lema II.8 Se $\left|s_{j}\right| \leq \log N$ para todo $j$, então quando $N \rightarrow \infty$ temos

$$
\mathbb{E}\left\{e^{i \frac{f_{N}\left(s_{X}\right)}{\sigma \sqrt{N}}}\right\}=\exp \left\{-\frac{1}{2} \sum_{i=1}^{k+1} \sum_{j=1}^{k+1} \rho_{i j} s_{i} s_{j}+o\left(\frac{1}{\sqrt{N}}\right)\right\}
$$

onde, para $1 \leq i, j \leq k+1$

$$
\rho_{i j}=\frac{1}{\sigma^{2} N} \sum_{X \subset[0, N]} \sum_{\mathcal{S}_{X}} \bar{\varphi}^{T}\left(\mathcal{S}_{X}\right) e^{-\left(\beta-\beta_{0}\right)\left|\mathcal{S}_{X}\right|}\left(\sum_{\xi \in X} g_{i}\left(\mathcal{S}_{\xi}\right) \delta \mathcal{S}_{\xi}\right)\left(\sum_{\xi \in X} g_{j}\left(\mathcal{S}_{\xi}\right) \delta \mathcal{S}_{\xi}\right) .
$$

Considerando a matriz $\Sigma_{N} \equiv\left(\rho_{i j}\right)$ de ordem $(k+1) \times(k+1)$, temos que ela é definida positiva para $N$ suficientemente grande.

Prova do lema II.8: Como fizemos na prova do lema II.7, desenvolvendo $\left(e^{i \frac{f_{N}\left(s_{X}\right)}{\sigma \sqrt{N}}}-1\right)$ em série de Taylor e lembrando que a função característica de distribuições simétricas em torno do zero é real, temos que

$$
\begin{aligned}
& \mathbb{E}\left\{e^{i \frac{f_{N}\left(\mathcal{S}_{X}\right)}{\sigma \sqrt{N}}}\right\}=\exp \left\{\sum_{X \subset[0, N]} \sum_{\mathcal{S}_{X}} \bar{\varphi}^{T}\left(\mathcal{S}_{X}\right) e^{-\left(\beta-\beta_{0}\right)\left|\mathcal{S}_{X}\right|}\left(e^{i \frac{f_{N}\left(S_{X}\right)}{\sigma \sqrt{N}}}-1\right)\right\} \\
& =\exp \left\{\sum_{X \subset[0, N]} \sum_{\mathcal{S}_{X}} \bar{\varphi}^{T}\left(\mathcal{S}_{X}\right) e^{-\left(\beta-\beta_{0}\right)\left|\mathcal{S}_{X}\right|}\left(-\frac{\left(f_{N}\left(\mathcal{S}_{X}\right)\right)^{2}}{2 \sigma^{2} N}+\frac{\left(f_{N}\left(\mathcal{S}_{X}\right)\right)^{4}}{24 \sigma^{4} N^{2}} \mathcal{I}\right)\right\},
\end{aligned}
$$

$\operatorname{com}|\mathcal{I}| \leq 1$.

Observamos que

$$
\begin{aligned}
\left(f_{N}\left(\mathcal{S}_{X}\right)\right)^{2} & =\left(\sum_{j=1}^{k+1} s_{j} \sum_{\xi \in X} g_{j}\left(\mathcal{S}_{\xi}\right) \delta \mathcal{S}_{\xi}\right)\left(\sum_{j=1}^{k+1} s_{j} \sum_{\xi \in X} g_{j}\left(\mathcal{S}_{\xi}\right) \delta \mathcal{S}_{\xi}\right) \\
& =\sum_{i=1}^{k+1} \sum_{j=1}^{k+1} s_{i} s_{j}\left(\sum_{\xi \in X} g_{i}\left(\mathcal{S}_{\xi}\right) \delta \mathcal{S}_{\xi}\right)\left(\sum_{\xi \in X} g_{j}\left(\mathcal{S}_{\xi}\right) \delta \mathcal{S}_{\xi}\right)
\end{aligned}
$$

e para $i \neq j,\left(\sum_{\xi \in X} g_{i}\left(\mathcal{S}_{\xi}\right) \delta \mathcal{S}_{\xi}\right)\left(\sum_{\xi \in X} g_{j}\left(\mathcal{S}_{\xi}\right) \delta \mathcal{S}_{\xi}\right)=0$ se $X \subset\left[a_{l-1}+1, a_{l}\right]$ para algum $l$. 
Para o termo envolvendo $\left(f_{N}\left(\mathcal{S}_{X}\right)\right)^{4}$ obtemos uma expressão análoga a de cima. Usando o lema II.6 e considerando $\left|s_{j}\right| \leq \log N$ temos a seguinte contribuição, quando $N \rightarrow \infty$ :

$$
\left|\frac{1}{24 \sigma^{4} N^{2}} \sum_{X \subset[0, N]} \sum_{\mathcal{S}_{X}} \bar{\varphi}^{T}\left(\mathcal{S}_{X}\right) e^{-\left(\beta-\beta_{0}\right)\left|\mathcal{S}_{X}\right|}\left(f_{N}\left(\mathcal{S}_{X}\right)\right)^{4} \mathcal{I}\right| \leq \frac{1}{24 \sigma^{4}} \frac{(\log N)^{4}}{N}\left(E_{4}+o(1)\right) .
$$

Assim (II.45) fica da forma

$$
\begin{gathered}
\exp \left\{-\frac{1}{2} \sum_{i=1}^{k+1} \sum_{j=1}^{k+1} \frac{1}{\sigma^{2} N} \sum_{X \subset[0, N]} \sum_{\mathcal{S}_{X}} \bar{\varphi}^{T}\left(\mathcal{S}_{X}\right) e^{-\left(\beta-\beta_{0}\right)\left|\mathcal{S}_{X}\right|} s_{i} s_{j}+\frac{1}{24 \sigma^{4}} \frac{(\log N)^{4}}{N}\left(E_{4}+o(1)\right)\right\} \\
=\exp \left\{-\frac{1}{2} \sum_{i=1}^{k+1} \sum_{j=1}^{k+1} \rho_{i j} s_{i} s_{j}+o\left(\frac{1}{\sqrt{N}}\right)\right\} \text { quando } N \rightarrow \infty
\end{gathered}
$$

Além disso, quando $N \rightarrow \infty$, por (II.42), (II.43) e lema II.6 segue que:

$$
\begin{aligned}
\rho_{i j} & =\frac{1}{\sigma^{2} N} \sum_{X \subset[0, N]} \sum_{\mathcal{S}_{X}} \bar{\varphi}^{T}\left(\mathcal{S}_{X}\right) e^{-\left(\beta-\beta_{0}\right)\left|\mathcal{S}_{X}\right|}\left(\sum_{\xi \in X} g_{i}\left(\mathcal{S}_{\xi}\right) \delta \mathcal{S}_{\xi}\right)\left(\sum_{\xi \in X} g_{j}\left(\mathcal{S}_{\xi}\right) \delta \mathcal{S}_{\xi}\right) \\
& = \begin{cases}\frac{\left(t_{j}-t_{j-1}\right)}{\sigma^{2}} \sigma^{2}+o(1) & \text { se } i=j \\
o(1) & \text { se } i \neq j .\end{cases}
\end{aligned}
$$

O fato de $\Sigma_{N}$ ser definida positiva segue de que definindo $\tau=\max _{1 \leq i \leq k+1} \sum_{\substack{1 \leq j \leq k+1 \\ j \neq i}}\left|\rho_{i j}\right|$, então a forma quadrática abaixo, para $\boldsymbol{y}^{\prime}=\left(y_{1}, \ldots, y_{k+1}\right) \in \mathbb{R}^{k+1}$ :

$$
y^{\prime} \Sigma_{N} y \geq \sum_{j=1}^{k+1}\left(\rho_{j j}-\tau\right) y_{j}^{2}>0
$$

pois $\min _{1 \leq j \leq k+1}\left(\rho_{j j}-\tau\right)>0$ se $N$ é suficientemente grande.

Portanto podemos expressar o integrando na fórmula de inversão como:

$$
\exp \left\{-\frac{1}{2} \boldsymbol{s}^{\prime} \boldsymbol{\Sigma}_{N} \boldsymbol{s}+o\left(\frac{1}{\sqrt{N}}\right)-i \frac{\boldsymbol{m}^{\prime} \boldsymbol{s}}{\sigma \sqrt{N}}\right\}
$$

$\operatorname{com} s^{\prime}=\left(s_{1}, \ldots, s_{k+1}\right)$ e $m^{\prime}=\left(m_{1}, m_{2}-m_{1}, \ldots, m_{j}-m_{j-1}, \ldots,-m_{k}\right)$.

Expandindo $e^{o\left(\frac{1}{\sqrt{N}}\right)}$, temos que quando $N \rightarrow \infty$ : 


$$
\begin{aligned}
& \left(\frac{1}{2 \pi \sigma \sqrt{N}}\right)^{k+1} \int \cdots \int \exp \left\{-\frac{1}{2} \boldsymbol{s}^{\prime} \boldsymbol{\Sigma}_{N} \boldsymbol{s}+o\left(\frac{1}{\sqrt{N}}\right)-i \frac{\boldsymbol{m}^{\prime} \boldsymbol{s}}{\sigma \sqrt{N}}\right\} d s_{1} \ldots d s_{k+1} \\
& =\left(\frac{1}{2 \pi \sigma \sqrt{N}}\right)^{k+1} \int \cdots \int \exp \left\{-\frac{1}{2} \boldsymbol{s}^{\prime} \boldsymbol{\Sigma}_{N} \boldsymbol{s}-i \frac{\boldsymbol{m}^{\prime} \boldsymbol{s}}{\sigma \sqrt{N}}\right\} d s_{1} \ldots d s_{k+1}+o\left(\frac{1}{N^{\frac{k+1}{2}+\frac{1}{2}}}\right) .
\end{aligned}
$$

Sendo $\Sigma_{N}$ definida positiva, então ela tem inversa $\Sigma_{N}^{\prime}$, logo quando $N \rightarrow \infty$

$$
\begin{gathered}
\left(\frac{1}{2 \pi \sigma \sqrt{N}}\right)^{k+1} \int_{\left|s_{j}\right| \leq \log N, \forall j}\left\{\mathbb{E}\left\{e^{i \frac{f_{N}\left(s_{X}\right)}{\sigma \sqrt{N}}}\right\} \prod_{j=1}^{k+1} e^{-i s_{j} \frac{\left(m_{j}-m_{j-1}\right)}{\sigma \sqrt{N}}}\right\} d s_{1} \ldots d s_{k+1} \\
=\frac{1}{\sqrt{\left(2 \pi \sigma^{2} N\right)^{k+1} \operatorname{det} \Sigma_{N}}} \exp \left\{-\frac{1}{2 \sigma^{2} N} \boldsymbol{m}^{\prime} \Sigma_{N}^{-1} \boldsymbol{m}\right\}+o\left(\frac{1}{N^{\frac{k+2}{2}}}\right),
\end{gathered}
$$

onde $\operatorname{det} \boldsymbol{\Sigma}_{N}$ denota o determinante da matriz $\boldsymbol{\Sigma}_{N}$. Note que obtemos a densidade de uma normal multivariada com média $\boldsymbol{m}$ e matriz de covariância $\boldsymbol{\Sigma}_{N}$ (ver, por exemplo [Jam], cap. 7).

Para terminar a prova do teorema II.1 basta observar que quando $N \rightarrow \infty$

$$
\Sigma_{N} \longrightarrow\left(\begin{array}{ccccc}
t_{1} & 0 & \cdots & 0 & 0 \\
0 & t_{2}-t_{1} & \cdots & 0 & 0 \\
\vdots & \vdots & \ddots & \vdots & \vdots \\
0 & 0 & \cdots & t_{k}-t_{k-1} & 0 \\
0 & 0 & \cdots & 0 & 1-t_{k}
\end{array}\right)
$$

e $P\left(\delta \mathcal{S}_{X}=0\right)=\frac{1}{2 \pi \sigma \sqrt{N}}+o\left(\frac{1}{N}\right)$

Portanto, como a probabilidade condicional é a integral em $\left[T_{j} \sigma \sqrt{N}, T_{j}^{\prime} \sigma \sqrt{N}\right]$, $j=1, \ldots, k$ do quociente de (II.46) com a expressão acima, obtemos o resultado desejado. 


\section{Apêndice A}

Neste apêndice apresentamos a definição do movimento Browniano e da ponte Browniana, bem como alguns teoremas clássicos de convergência que nos auxiliarão a provar a proposição I.1.

\section{A-1 Definições e Resultados}

Um elemento aleatório $X$ de $C$, espaço das funções contínuas, é Gaussiano se todas as suas distribuições finito-dimensionais são normais. A distribuição em $C$ de um elemento Gaussiano é completamente especificada pelas médias $\mathbb{E}(X(t))$ e pelos momentos produto $\mathbb{E}(X(s) X(t)), s, t \geq 0$, porque eles determinam as distribuições finito-dimensionais.

Definição A.1 O movimento Browniano $\mathcal{W}$ é um elemento Gaussiano de $C$, cujos momentos são dados por:

(i) $\mathcal{W}_{0} \equiv 0$,

(ii) $\mathbb{E}\left(\mathcal{W}_{t}\right)=0$ para todo $t>0$,

(iii) $\mathbb{E}\left(\mathcal{W}_{s} \mathcal{W}_{t}\right)=s$ se $0 \leq s \leq t$.

No decorrer deste texto usaremos tanto $\mathcal{W}_{t}$ como $\mathcal{W}(t)$ para denotar o movimento Browniano no instante $t$. 
Definição A.2 A ponte Browniana $\mathcal{B}$ é um elemento Gaussiano de $C[0,1]$, cujos momentos são dados por:

(i) $\mathcal{B}_{0} \equiv 0$ e $\mathcal{B}_{1} \equiv 0$,

(ii) $\mathbb{E}\left(\mathcal{B}_{t}\right)=0$ para todo $0 \leq t \leq 1$,

(iii) $\mathbb{E}\left(\mathcal{B}_{s} \mathcal{B}_{t}\right)=s(1-t)$ se $0 \leq s \leq t \leq 1$.

Um modo de se obter a ponte Browniana, sem precisar construir o processo passo a passo, é através da seguinte transformação do movimento Browniano: $\mathcal{B}_{t}=\mathcal{W}_{t}-t \mathcal{W}_{1}, \quad 0 \leq t \leq 1$.

A partir da definição da ponte Browniana, temos que suas distribuições finitodimensionais, para reais $T_{j}<T_{j}^{\prime}, j=1, \ldots, k$, são dadas por:

$$
\begin{aligned}
P\left(\mathcal{B}_{t_{1}} \in\left[T_{1}, T_{1}^{\prime}\right],\right. & \left.\ldots, \mathcal{B}_{t_{k}} \in\left[T_{k}, T_{k}^{\prime}\right]\right) \\
= & \sqrt{2 \pi} \int_{T_{1}}^{T_{1}^{\prime}} d x_{1} p\left(t_{1}, x_{1}\right) \int_{T_{2}}^{T_{2}^{\prime}} d x_{2} p\left(t_{2}-t_{1}, x_{2}-x_{1}\right) \times \ldots \\
& \ldots \times \int_{T_{k}}^{T_{k}^{\prime}} d x_{k} p\left(t_{k}-t_{k-1}, x_{k}-x_{k-1}\right) p\left(1-t_{k},-x_{k}\right) .
\end{aligned}
$$

onde $p(t, x)=(2 \pi t)^{-1 / 2} \exp \left\{-x^{2} / 2 t\right\}$ e $0 \leq t_{1}<t_{2}<\ldots<t_{k} \leq 1$.

O teorema abaixo estabelece a convergência fraca da soma de variáveis aleatórias independentes e com mesma distribuição, para o movimento Browniano.

Teorema A.1 (Teorema de Donsker) Sejam $\left\{\xi_{i}\right\}$ uma seqüência de variáveis aleatórias independentes e identicamente distribuídas em $(\Omega, \mathcal{F}, P)$, com média zero e variância finita e positiva $\sigma^{2}$; e seja $S_{n}=\xi_{1}+\ldots+\xi_{n}$ as somas parciais. Então temos que

$$
X_{n}(t, \omega)=\frac{1}{\sigma \sqrt{n}} S_{\lfloor n t\rfloor}(\omega), \quad t \in[0,1]
$$

define uma função aleatória em $D[0,1]$ e converge fracamente para o movimento Browniano na topologia de Skorohod.

A prova desse teorema se encontra na seção 16 de [Bil]. 
Teorema A.2 (Teorema do Limite Local) Seja $\left\{X_{n}\right\}$ uma seqüência de variáveis aleatórias independentes e identicamente distribuídas com média $\alpha$ e variância finita e positiva $\sigma^{2}$, assumindo apenas valores inteiros. Considere que $S_{n}=X_{1}+\ldots+X_{n}$, as somas parciais, também assumem apenas valores inteiros, isto é, $\sum_{k \in \boldsymbol{Z}} P\left(S_{n}=k\right)=1$. Então denotando $z_{n k}=(k-\alpha n) / \sigma \sqrt{n}$ temos que para

$$
\sup _{k}\left|\sigma \sqrt{n} P\left(S_{n}=k\right)-\frac{1}{\sqrt{2 \pi}} \exp \left\{-\frac{z_{n k}^{2}}{2}\right\}\right| \longrightarrow 0 \quad \text { quando } n \rightarrow \infty
$$

valer para $-\infty<k<\infty$, é necessário e suficiente que, o maior divisor comum das diferenças pareadas de todos os valores de $X_{1}$ assumidos com probabilidade positiva, seja igual a um.

A prova consta em Gnedenko e Kolmogorov [GK] §49 ou Petrov [Pet].

Em seguida apresentamos um teorema que estabelece condições para preservar massa. Note que uma seqüência $\left\{X_{n}\right\}$ de elementos aleatórios de $D[0,1]$, por definição, preserva massa quando a seqüência $\left\{P_{n}\right\}$ de distribuições correspondente preserva massa; onde denotamos $P_{n}(X \in A) \stackrel{\text { not }}{=} P\left(X_{n} \in A\right)$. (ver teorema 15.3 de [Bil]).

\section{Teorema A.3 (Condições necessárias e suficientes para preservar massa)}

Uma seqüência $\left\{P_{n}\right\}$ de medidas de probabilidade em $(D, \mathcal{D})$ preserva massa se e somente se satisfaz as duas condições abaixo:

(i) Para cada $\eta$ positivo, existe um a tal que

$$
P_{n}\left(\sup _{s \leq 1}|x(s)|>a\right) \leq \eta \quad \text { para } n \geq 1
$$

(ii) Para cada $\varepsilon$ e $\eta$ positivos, existe um $\delta, 0<\delta<1$, e um inteiro $n_{0}$ tais que

$$
\begin{aligned}
P_{n}\left(w_{x}^{\prime \prime}(\delta, 1) \geq \varepsilon\right) & \leq \eta \quad \text { para } n \geq n_{0}, \\
P_{n}\left(w_{x}[0, \delta) \geq \varepsilon\right) & \leq \eta \quad \text { para } n \geq n_{0} \quad \text { e } \\
P_{n}\left(w_{x}[1-\delta, 1) \geq \varepsilon\right) & \leq \eta \quad \text { para } n \geq n_{0} .
\end{aligned}
$$

onde $w_{x}$ e $w_{x}^{\prime \prime}$ são funcionais em $D[0,1]$ definidos, para cada $\delta>0$ e $r \in(0,1]$, por:

$$
\begin{aligned}
w_{x}[0, \delta) & =\sup \left\{\left|x\left(s_{1}\right)-x\left(s_{2}\right)\right| ; 0 \leq s_{1}, s_{2}<\delta\right\}, \\
w_{x}[1-\delta, 1) & =\sup \left\{\left|x\left(s_{1}\right)-x\left(s_{2}\right)\right| ; 1-\delta \leq s_{1}, s_{2}<1\right\} \text { e } \\
w_{x}^{\prime \prime}(\delta, r) & =\sup \left\{\min \left(\left|x(s)-x\left(s_{1}\right)\right|,\left|x\left(s_{2}\right)-x(s)\right|\right) ; 0 \leq s_{1} \leq s \leq s_{2} \leq r, s_{2}-s_{1} \leq \delta\right\} .
\end{aligned}
$$




\section{Apêndice B}

Neste apêndice apresentamos as provas de alguns resultados relativos ao segundo capítulo. A primeira seção corresponde à expansão em conglomerados de contornos e a segunda à expansão do gás de formas.

\section{B-1 Expansão em Conglomerados de Contornos}

Prova de (II.5):

Se $\varphi_{1}, \varphi_{2} \in \mathcal{D}_{\theta}$ então $\sum_{\Gamma}\left|\varphi_{i}(\Gamma) \chi_{\theta}(\Gamma)\right|<\infty, i=1,2$. Logo,

$$
\begin{aligned}
\sum_{\Gamma}\left|\left(\varphi_{1} * \varphi_{2}\right)(\Gamma) \chi_{\theta}(\Gamma)\right| & =\sum_{\Gamma}\left|\sum_{\Gamma_{1} \subset \Gamma} \varphi_{1}\left(\Gamma_{1}\right) \varphi_{2}\left(\Gamma \backslash \Gamma_{1}\right) \chi_{\theta}\left(\Gamma_{1}\right) \chi_{\theta}\left(\Gamma \backslash \Gamma_{1}\right)\right| \\
& \leq \sum_{\Gamma} \sum_{\Gamma_{1} \subset \Gamma}\left|\varphi_{1}\left(\Gamma_{1}\right) \chi_{\theta}\left(\Gamma_{1}\right)\right|\left|\varphi_{2}\left(\Gamma \backslash \Gamma_{1}\right) \chi_{\theta}\left(\Gamma \backslash \Gamma_{1}\right)\right| \\
& \leq\left(\sum_{\Gamma}\left|\varphi_{1}(\Gamma) \chi_{\theta}(\Gamma)\right|\right)\left(\sum_{\Gamma}\left|\varphi_{2}(\Gamma) \chi_{\theta}(\Gamma)\right|\right)<\infty \\
\Longrightarrow\left(\varphi_{1} * \varphi_{2}\right) \in \mathcal{D}_{\theta} \quad \mathrm{e} & \\
\left\langle\chi_{\theta}, \varphi_{1} * \varphi_{2}\right\rangle & =\sum_{\Gamma}\left(\varphi_{1} * \varphi_{2}\right)(\Gamma) \chi_{\theta}(\Gamma)=\sum_{\Gamma} \sum_{\Gamma 1} \varphi_{1}\left(\Gamma_{1}\right) \varphi_{2}\left(\Gamma \backslash \Gamma_{1}\right) \chi_{\theta}\left(\Gamma_{1}\right) \chi_{\theta}\left(\Gamma \backslash \Gamma_{1}\right) \\
& =\sum_{\Gamma_{1}} \varphi_{1}\left(\Gamma_{1}\right) \chi_{\theta}\left(\Gamma_{1}\right) \sum_{\Gamma \supset \Gamma_{1}} \varphi_{2}\left(\Gamma \backslash \Gamma_{1}\right) \chi_{\theta}\left(\Gamma \backslash \Gamma_{1}\right) \\
& =\sum_{\Gamma_{1}} \varphi_{1}\left(\Gamma_{1}\right) \chi_{\theta}\left(\Gamma_{1}\right) \sum_{\Gamma_{2}} \varphi_{2}\left(\Gamma_{2}\right) \chi_{\theta}\left(\Gamma_{2}\right)=\left\langle\chi_{\theta}, \varphi_{1}\right\rangle\left\langle\chi_{\theta}, \varphi_{2}\right\rangle
\end{aligned}
$$


Prova das propriedades (i), (ii) e (iii) da definição II.5

(i) $D_{\gamma}\left(\varphi_{1} * \varphi_{2}\right)(\Gamma)=\left(\varphi_{1} * \varphi_{2}\right)(\Gamma \cup \Upsilon)$

$$
\begin{aligned}
& =\sum_{\Gamma_{1} \subset \Gamma}\left\{\varphi_{1}\left(\Gamma_{1} \cup \gamma\right) \varphi_{2}\left(\Gamma \backslash \Gamma_{1}\right)-\varphi_{1}\left(\Gamma_{1}\right) \varphi_{2}\left(\left(\Gamma \backslash \Gamma_{1}\right) \cup \gamma\right)\right\} \\
& =\sum_{\Gamma_{1} \subset \Gamma}\left(D_{\gamma} \varphi_{1}\right)\left(\Gamma_{1}\right) \varphi_{2}\left(\Gamma \backslash \Gamma_{1}\right)+\sum_{\Gamma_{1} \subset \Gamma} \varphi_{1}\left(\Gamma_{1}\right)\left(D_{\gamma} \varphi_{2}\right)\left(\Gamma \backslash \Gamma_{1}\right) \\
& =\left\{\varphi_{2} *\left(D_{\gamma} \varphi_{1}\right)\right\}(\Gamma)+\left\{\varphi_{1} *\left(D_{\gamma} \varphi_{2}\right)\right\}(\Gamma) .
\end{aligned}
$$

(ii) $\quad D_{\gamma}(\operatorname{Exp} \varphi)(\Gamma)=(\operatorname{Exp} \varphi)(\Gamma \cup \gamma)=\sum_{n \geq 0} \frac{1}{n !} \varphi^{n}(\Gamma \cup \gamma)$

$$
\begin{aligned}
& =\sum_{n \geq 0} \frac{1}{n !} \sum_{\Gamma_{1} \cup \ldots \cup \Gamma_{n}=\Gamma}\left(\sum_{i=1}^{n} \varphi\left(\Gamma_{i} \cup \gamma\right) \prod_{\substack{j=1 \\
j \neq i}}^{n} \varphi\left(\Gamma_{j}\right)\right) \\
& =\sum_{n \geq 0} \frac{1}{n !} \sum_{i=1}^{n} \sum_{\Gamma_{1} \cup \ldots \cup \Gamma_{n}=\Gamma}\left(D_{\gamma} \varphi\right)\left(\Gamma_{i}\right) \prod_{\substack{j \neq i \\
j}} \varphi\left(\Gamma_{j}\right)=\sum_{n \geq 0} \frac{1}{n !} n\left(\left(D_{\gamma} \varphi\right) * \varphi^{n-1}\right)(\Gamma) \\
& =\left\{\left(D_{\gamma} \varphi\right) *\left(\sum_{n \geq 1} \frac{1}{(n-1) !} \varphi^{n-1}\right)\right\}(\Gamma)=\left(\left(D_{\gamma} \varphi\right) * \operatorname{Exp} \varphi\right)(\Gamma) .
\end{aligned}
$$

(iii) Para $\varphi \in \mathcal{F}_{1}$, considere $\psi \in \mathcal{F}_{0}$ tal que $\psi=\log \varphi$ e $\varphi=\operatorname{Exp} \psi$. Usando a. propriedade (ii) temos

$$
\begin{aligned}
\varphi^{-1} *\left(D_{\gamma} \varphi\right) & =\varphi^{-1} *\left(D_{\gamma}(\operatorname{Exp} \psi)\right)=\varphi^{-1} * D_{\gamma} \psi * \operatorname{Exp} \psi=\varphi^{-1} * D_{\gamma} \psi * \varphi \\
& =D_{\gamma} \psi=D_{\gamma}(\log \varphi)
\end{aligned}
$$

Prova do Lema II.1

$$
\begin{aligned}
\left\langle\chi_{\theta}, \operatorname{Exp} \varphi\right\rangle & \stackrel{\text { not }}{=} \sum_{\Gamma}(\operatorname{Exp} \varphi)(\Gamma) \chi_{\theta}(\Gamma)=\sum_{\Gamma} \sum_{n \geq 0} \frac{1}{n !} \varphi^{n}(\Gamma) \chi_{\theta}(\Gamma) \\
& =\sum_{n \geq 0} \frac{1}{n !} \sum_{\Gamma} \varphi^{n}(\Gamma) \chi_{\theta}(\Gamma)=\sum_{n \geq 0} \frac{1}{n !}\left\langle\chi_{\theta}, \varphi^{n}\right\rangle=\sum_{n \geq 0} \frac{1}{n !}\left\{\left\langle\chi_{\theta}, \varphi\right\rangle\right\}^{n} \\
& =\exp \left\{\left\langle\chi_{\theta}, \varphi\right\rangle\right\}=\exp \left\{\sum_{\Gamma} \varphi(\Gamma) \chi_{\theta}(\Gamma)\right\}
\end{aligned}
$$




\section{Prova do Lema II.2}

Seja $\gamma \cup \Gamma$ um conjunto de contornos compatíveis e $\varphi$ como em (II.6):

$$
\begin{aligned}
\Delta_{\gamma \cup \Gamma}(\Upsilon) & =\left(\varphi^{-1} *\left(D_{\gamma \cup \Gamma} \varphi\right)\right)(\Upsilon)=\sum_{\substack{\Upsilon_{1} \cup \Upsilon_{2}=\Upsilon \\
\Upsilon_{1} \cap \Upsilon_{2}=\emptyset}} \varphi^{-1}\left(\Upsilon_{1}\right) \varphi\left(\gamma \cup \Gamma \cup \Upsilon_{2}\right) \\
& =\sum_{\substack{\Upsilon_{1} \cup \Upsilon_{2}=\Upsilon \\
\Upsilon_{2} \cap(\gamma \cup \Gamma)=\emptyset}} \varphi^{-1}\left(\Upsilon_{1}\right) \exp \left\{-\beta\left(|\Gamma|+|\gamma|+\left|\Upsilon_{2}\right|\right)\right\} \\
& =e^{-\beta|\gamma|} \sum_{\substack{\Upsilon_{1} \cup \Upsilon_{2}=\Upsilon \\
\Upsilon_{2} \cap(\gamma \cup \Gamma)=\emptyset}} \varphi^{-1}\left(\Upsilon_{1}\right) \varphi\left(\Gamma \cup \Upsilon_{2}\right),
\end{aligned}
$$

observamos que $\sum_{\substack{X_{1} \cup X_{2}=X \\ X_{2} \cap Y^{\prime}=\emptyset}}(\cdot)=\sum_{X_{1} \cup X_{2}=X}(\cdot)-\sum_{\substack{X_{1} \cup X_{2}=X \\ X_{2} \cap Y \neq \emptyset}}(\cdot)$ de modo que podemos escrever $\left\{X_{2} \cap Y \neq \emptyset\right\}=\bigcup_{i=1}^{\infty} A_{i}$ onde cada $A_{i}$ é um conjunto com $i$ elementos, resultante da intersecção de $X_{2}^{i=1} \operatorname{com} Y$. Pelo princípio da inclusão-exclusão temos que:

$$
\sum_{\substack{X_{1} \cup X_{2}=X \\ X_{2} \cap Y^{\prime} \neq \emptyset}}(\cdot)=\sum_{n \geq 1} \sum_{A: N(A)=n}(-1)^{n+1} \sum_{\substack{X_{1} \cup X_{2}=X \\ X_{2} \cap Y^{\prime}=A}}(\cdot)
$$

onde $N(A)$ é o número de elementos em $A$.

Esses resultados aplicados à soma em (B.1) nos fornecem:

$$
\begin{aligned}
\sum_{\substack{\Upsilon_{1} \cup \Upsilon_{2}=\Upsilon \\
\Upsilon_{2} \cap(\gamma \cup \Gamma)=\emptyset}} \varphi^{-1}\left(\Upsilon_{1}\right) \varphi\left(\Gamma \cup \Upsilon_{2}\right)= & \sum_{\Upsilon_{1} \cup \Upsilon_{2}} \varphi^{-1}\left(\Upsilon_{1}\right) \varphi\left(\Gamma \cup \Upsilon_{2}\right) \\
& -\sum_{n \geq 1} \sum_{P: N(P)=n}(-1)^{n+1} \sum_{\substack{\Upsilon_{1} \cup \Upsilon_{2}=\Upsilon \\
\Upsilon_{2} \cap(\gamma \cup)=P}} \varphi^{-1}\left(\Upsilon_{1}\right) \varphi\left(\Gamma \cup \Upsilon_{2}\right)
\end{aligned}
$$

Como $P \subset \Upsilon$ podemos fazer a seguinte transformação:

$$
\left\{\begin{array} { l } 
{ \Upsilon _ { 1 } = \Upsilon _ { 1 } ^ { \prime } } \\
{ \Upsilon _ { 2 } = \Upsilon _ { 2 } ^ { \prime } \cup P }
\end{array} \Longrightarrow \left\{\begin{array}{l}
\Upsilon_{1}^{\prime} \cup \Upsilon_{2}^{\prime}=\Upsilon \backslash P \quad \text { e } \\
P \cap(\gamma \cup \Gamma) \neq \emptyset
\end{array}\right.\right.
$$

no entanto, $\varphi$ definida em (II.6) só não se anula caso $\Gamma \cup \Upsilon_{2}=\Gamma \cup\left(\Upsilon_{2}^{\prime} \cup P\right)$ seja um conjunto nulo ou só contenha contornos compatíveis, de maneira que $\Gamma \cap P=\emptyset$. Porém, como $P \cap(\gamma \cup \Gamma) \neq \emptyset$, então obrigatoriamente $P \cap \gamma \neq \emptyset$. Logo, segue que 


$$
\begin{aligned}
\Delta_{\gamma \cup \Gamma}(\Upsilon)= & e^{-\beta|\gamma|}\left\{\sum_{\Upsilon_{1} \cup \Upsilon_{2}=\Upsilon} \varphi^{-1}\left(\Upsilon_{1}\right) \varphi\left(\Gamma \cup \Upsilon_{2}\right)\right. \\
& \left.-\sum_{\substack{n>1 \\
n=N(P)}} \sum_{\substack{P \subset \Upsilon \neq \emptyset \\
P \cap \gamma \neq \emptyset}}(-1)^{n+1} \sum_{\Upsilon_{1}^{\prime} \cup \Upsilon_{2}^{\prime}=\Upsilon \backslash P} \varphi^{-1}\left(\Upsilon_{1}^{\prime}\right) \varphi\left(\Gamma \cup P \cup \Upsilon_{2}^{\prime}\right)\right\} \\
= & e^{-\beta|\gamma|}\left\{\Delta_{\Gamma}(\Upsilon)+\sum_{\substack{\phi \neq P \subset \Upsilon \\
P \cap \gamma \neq \emptyset}}(-1)^{N(P)} \Delta_{\Gamma \cup P}(\Upsilon \backslash P)\right\} \\
= & e^{-\beta|\gamma|} \sum_{\substack{P \subset \Upsilon \\
P \cap \gamma \neq \emptyset}}(-1)^{N(P)} \Delta_{\Gamma \cup P}(\Upsilon \backslash P),
\end{aligned}
$$

onde $\overline{P \cap \gamma} \neq \emptyset$ significa que $P$ intersepta $\gamma$ ou $P=\emptyset$, e $\Gamma \cup P$ é um conjunto de contornos compatíveis.

\section{Prova da Proposição I.1:}

Prova de (1): Para $p$ fixado temos:

$$
\begin{aligned}
\sum_{\Gamma \ni p}\left|\varphi^{T}(\Gamma)\right| & \leq \sum_{\gamma \ni p} \sum_{\Gamma}\left|\varphi^{T}(\gamma \cup \Gamma)\right|=\sum_{\gamma \ni p} \sum_{\Gamma}\left|\Delta_{\gamma}(\Gamma)\right| \leq \sum_{\gamma \ni p} \sum_{m=1}^{\infty} \sum_{N(\Gamma)=m-1}\left|\Delta_{\gamma}(\Gamma)\right| \\
& \leq \sum_{\gamma \ni p} \sum_{m=1}^{\infty} \sup _{\gamma} \sum_{\Gamma: N(\Gamma)=m-1}\left|\Delta_{\gamma}(\Gamma)\right| e^{\frac{\beta}{2}|\gamma|} e^{-\frac{\beta}{2}|\gamma|} \leq \sum_{\gamma \ni p} \sum_{m=1}^{\infty} I_{m} e^{-\frac{\beta}{2}|\gamma|} \\
& \leq \sum_{\gamma \ni p} \sum_{m=1}^{\infty} e^{-\beta(m+1)} e^{-\frac{\beta}{2}|\gamma|} \leq \sum_{\gamma \ni p} e^{-\frac{\beta}{2}|\gamma|}\left(\frac{e^{-2 \beta}}{1-e^{-\beta}}\right) \leq\left(\frac{e^{-2 \beta}}{1-e^{-\beta}}\right) \sum_{l=4}^{\infty}\left(3 e^{-\frac{\beta}{2}}\right)^{l} \\
& =\delta(\beta) \text { se } \beta>2 \ln 6
\end{aligned}
$$

onde $\delta(\beta)$ converge exponencialmente para zero quando $\beta$ tende a infinito.

Prova de (2):

$$
\begin{aligned}
\sum_{\substack{\Gamma \ni \gamma \\
N(\Gamma)=n+1}}\left|\varphi^{T}(\Gamma)\right| & =\sum_{\substack{\Gamma \\
N(\Gamma)=n}}\left|\varphi^{T}(\Gamma \cup \gamma)\right|=\sum_{\substack{\Gamma(\Gamma)=n \\
N}}\left|\Delta_{\gamma}(\Gamma)\right| \leq I_{n} e^{-\frac{\beta}{2}|\gamma|} \\
& \leq\left(e^{-\beta}\right)^{n+1} e^{-\frac{\beta}{2}|\gamma|}=(\varpi(\beta))^{n+1} e^{-\frac{\beta}{2}|\gamma|} .
\end{aligned}
$$

Prova de (3): Por (II.9) temos que

$$
\varphi^{T}(\Gamma)=e^{-\beta|\Gamma|} \sum_{\substack{C \subset G \\ C \text { conexo }}}(-1)^{\# \text { arestas em } C}=e^{-\beta|\Gamma|} \tilde{\varphi}^{T}(\Gamma),
$$


com $\tilde{\varphi}^{T}(\Gamma)$ independente de $\beta$, dependendo apenas da geometria dos contornos. Além disso, se $\varphi^{T}(\Gamma) \neq 0$ então $\Gamma$ tem contornos incompatíveis formando um grupo conexo. Portanto, se $p \in \Gamma, d=d(p, Q)$ e $\Gamma \cap Q \neq \emptyset$ então $|\Gamma| \geq d$, logo considerando um $\beta_{0}>\beta$

$$
\begin{aligned}
\sum_{\substack{\Gamma \ni p \\
\Gamma \cap Q \neq 0}}\left|\varphi^{T}(\Gamma)\right| & \leq \sum_{\substack{\Gamma \ni p \\
|\Gamma| \geq d}}\left|\varphi^{T}(\Gamma)\right|=\sum_{\substack{\Gamma \ni p \\
|\Gamma| \geq d}} e^{-\beta|\Gamma|}\left|\tilde{\varphi}^{T}(\Gamma)\right| e^{ \pm \beta_{0}|\Gamma|} \\
& \leq e^{-\left(\beta-\beta_{0}\right) d} \sum_{\substack{\Gamma \ni p \\
|\Gamma| \geq d}}\left|e^{-\beta_{0}|\Gamma|} \tilde{\varphi}^{T}(\Gamma)\right| \leq e^{-\left(\beta-\beta_{0}\right) d} \sum_{\Gamma \ni p}\left|\varphi_{\beta_{0}}^{T}(\Gamma)\right| \\
& \leq e^{-\left(\beta-\beta_{0}\right) d} \delta\left(\beta_{0}\right),
\end{aligned}
$$

onde na última desigualdade utilizamos (1) da proposição II.1

Prova de (4): Usando o mesmo argumento que na prova de (3), temos que $\varphi^{T}(\Gamma)=e^{-\beta|\Gamma|} \tilde{\varphi}^{T}(\Gamma) \operatorname{com} \tilde{\varphi}^{T}(\Gamma)$ independente de $\beta$. Portanto, para $\beta>\beta_{0}>0$

$$
\Phi_{0}(Y)=\sum_{\Gamma \ni Y}\left|\varphi^{T}(\Gamma)\right|=\sum_{\Gamma \ni Y} e^{-\beta|\Gamma|}\left|\tilde{\varphi}^{T}(\Gamma)\right| e^{ \pm \beta_{0}|\Gamma|} .
$$

Seja $R_{0}$ um inteiro fixado arbitrariamente grande (por exemplo, $R_{0}=100$ ) então se $2 R_{0} e^{-\left(\beta-\beta_{0}\right)}<1$ e usando (2) da proposição II.1 obtemos

$$
\begin{aligned}
\sum_{Y \ni 0} R^{\operatorname{diam} Y} \Phi_{0}(Y) \leq \sum_{n \geq 0} \sum_{Y \ni 0} \sum_{\substack{\Gamma \ni Y \\
N(\Gamma)=n+1}} R^{\operatorname{diam} Y} e^{-\left(\beta-\beta_{0}\right)|\Gamma|} e^{-\beta_{0}|\Gamma|}\left|\tilde{\varphi}^{T}(\Gamma)\right| \\
=\sum_{n \geq 0} \sum_{\substack{\Gamma \ni 0 \\
N(\Gamma)=n+1}} \sum_{\substack{Y \in \Gamma \\
Y \rightarrow 0}} R^{\operatorname{diam} Y} e^{-\left(\beta-\beta_{0}\right)|\Gamma|} e^{-\beta_{0}|\Gamma|}\left|\tilde{\varphi}^{T}(\Gamma)\right| \\
\leq \sum_{n \geq 0} \sum_{\substack{\Gamma \ni 0 \\
N(\Gamma)=n+1}}\left(2 R_{0}\right)^{|\Gamma|} e^{-\left(\beta-\beta_{0}\right)|\Gamma|} e^{-\beta_{0}|\Gamma|}\left|\tilde{\varphi}^{T}(\Gamma)\right| \\
\leq \sum_{n \geq 0} \sum_{\gamma \ni 0} \sum_{\substack{\Gamma \ni \gamma \\
N(\Gamma)=n+1}}\left(2 R_{0} e^{-\beta-\beta_{0}}\right)^{|\Gamma|} e^{-\beta_{0}|\Gamma|}\left|\tilde{\varphi}^{T}(\Gamma)\right| \\
\leq 2 R_{0} e^{-\left(\beta-\beta_{0}\right)} \sum_{\gamma \ni 0} \sum_{n \geq 0} \varpi\left(\beta_{0}\right)^{n+1} e^{-\frac{\beta_{0}}{2}|\gamma|}=2 R_{0} e^{-\left(\beta_{0}-\beta\right)} \sum_{\gamma \ni 0} e^{-\frac{\beta_{0}}{2}|\gamma|} \frac{\varpi\left(\beta_{0}\right)}{1-\varpi\left(\beta_{0}\right)} \\
\leq 2 R_{0} e^{-\left(\beta-\beta_{0}\right)} \frac{\varpi\left(\beta_{0}\right)}{1-\varpi\left(\beta_{0}\right)} \frac{9 e^{-2 \beta_{0}}}{1-3 e^{-\frac{\beta_{0}}{2}}}=\psi(\beta),
\end{aligned}
$$


$\operatorname{com} \psi(\beta) \stackrel{\beta \rightarrow \infty}{\longrightarrow} 0$ exponencialmente.

Observamos que (B.2) é válida para $1 \leq R \leq R_{0}$ e $\beta$ suficientemente grande. Tomando $R=1$ em (B.2) temos que $\sum_{Y_{\ni 0}} \Phi_{0}(Y) \leq \psi(\beta)<\infty$ e tomando $R=R_{0}$ temos que

$$
\sum_{Y \ni 0, \operatorname{diam} Y \geq r} \Phi_{0}(Y) R_{0}^{\operatorname{diam} Y} \leq \psi(\beta) \Longrightarrow \sum_{Y \ni 0, \operatorname{diam} Y \geq r} \Phi_{0}(Y) \leq R_{0}^{-r} \psi(\beta)
$$

\section{B-2 Expansão em Conglomerados do Gás de Formas}

A partir da definição II.9 podemos obter as relações abaixo que nos serão úteis na prova do lema II.5. Considere o conjunto de aglomerados $X^{(1)}=X \backslash\left\{\xi_{1}\right\}=\left\{\xi_{2}, \ldots, \xi_{n}\right\}$ e $Y$ tal que $X \cap Y=\emptyset$, então de (II.19) e da definição II.9 resultam:

$$
\begin{aligned}
U\left(\mathcal{S}_{X \cup Y}\right) & =U\left(\mathcal{S}_{X} \cup \mathcal{S}_{Y}\right)=U_{1}\left(\mathcal{S}_{X}\right)+U\left(\mathcal{S}_{X^{(1)}} \cup \mathcal{S}_{Y}\right)+I_{1}\left(\mathcal{S}_{X}, \mathcal{S}_{Y^{\prime}}\right) \\
e^{-I_{1}\left(\mathcal{S}_{X}, \mathcal{S}_{Y}\right)} & =1+\sum_{\emptyset \neq P \subset Y} K_{1}\left(\mathcal{S}_{X}, \mathcal{S}_{P}\right)=\sum_{P \subset Y} K_{1}\left(\mathcal{S}_{X}, \mathcal{S}_{P}\right)
\end{aligned}
$$

Prova de (B.3):

$$
\begin{aligned}
U\left(\mathcal{S}_{X} \cup \mathcal{S}_{Y^{\prime}}\right) & =\sum_{T \subset X^{\prime} \cup Y^{\prime}} \Phi\left(\mathcal{S}_{T}\right)=\sum_{P \subset Y^{\prime}} \sum_{T \subset X} \Phi\left(\mathcal{S}_{P} \cup \mathcal{S}_{T}\right)=\sum_{P \subset Y^{\prime}} \sum_{T \subset X^{(1)} \cup \xi_{1}} \Phi\left(\mathcal{S}_{P} \cup \mathcal{S}_{T}\right) \\
& =\sum_{P \subset Y}\left\{\sum_{T \subset X^{(1)}} \Phi\left(\mathcal{S}_{P} \cup \mathcal{S}_{T}\right)+\sum_{T \subset X^{(1)}} \Phi\left(\mathcal{S}_{P} \cup \mathcal{S}_{T} \cup \mathcal{S}_{\xi_{1}}\right)\right\} \\
& =U\left(\mathcal{S}_{X^{(1)}} \cup \mathcal{S}_{Y^{\prime}}\right)+\left\{\sum_{T \subset X^{(1)}} \Phi\left(\mathcal{S}_{T} \cup \mathcal{S}_{\xi_{1}}\right)+\sum_{\substack{\emptyset \neq P \subset Y^{\prime} \\
T \subset X^{(1)}}} \Phi\left(\mathcal{S}_{P} \cup \mathcal{S}_{T} \cup \mathcal{S}_{\xi_{1}}\right)\right\} \\
& =U\left(\mathcal{S}_{X^{(1)}} \cup \mathcal{S}_{Y^{\prime}}\right)+\sum_{\xi_{1} \in T \subset X} \Phi\left(\mathcal{S}_{T}\right)+\sum_{\substack{\emptyset \neq P \subset Y \\
\xi \in T \subset X}} \Phi\left(\mathcal{S}_{P} \cup \mathcal{S}_{T}\right) \\
& =U\left(\mathcal{S}_{X^{(1)}} \cup \mathcal{S}_{Y^{\prime}}\right)+U_{1}\left(\mathcal{S}_{X}\right)+I_{1}\left(\mathcal{S}_{X}, \mathcal{S}_{Y^{\prime}}\right) .
\end{aligned}
$$


Prova de (B.4):

$$
\begin{aligned}
e^{I_{1}\left(\mathcal{S}_{X}, \mathcal{S}_{Y}\right)} & =\exp \left\{-\sum_{\emptyset \neq P \subset Y} W_{1}\left(\mathcal{S}_{X}, \mathcal{S}_{P}\right)\right\}=\prod_{\emptyset \neq P \subset Y} e^{-W_{1}\left(\mathcal{S}_{X}, \mathcal{S}_{P}\right)} \\
& =\prod_{\emptyset \neq P \subset Y}\left[1+\left(e^{-W_{1}\left(\mathcal{S}_{X}, \mathcal{S}_{P}\right)}-1\right)\right]=1+\sum_{\emptyset \neq P \subset Y} \sum_{\substack { n \geq 1 \\
\begin{subarray}{c}{\left\{T_{1}, \cdots, T_{n}\right\} \\
\cup T_{i}=P{ n \geq 1 \\
\begin{subarray} { c } { \{ T _ { 1 } , \cdots , T _ { n } \} \\
\cup T _ { i } = P } }\end{subarray}} \prod_{j=1}^{n}\left(e^{-W_{1}\left(\mathcal{S}_{X}, \mathcal{S}_{T_{j}}\right)}-1\right) \\
& =1+\sum_{\emptyset \neq P \subset Y} K_{1}\left(\mathcal{S}_{X}, \mathcal{S}_{P}\right)=\sum_{P \subset Y} K_{1}\left(\mathcal{S}_{X}, \mathcal{S}_{P}\right) .
\end{aligned}
$$

\section{Prova do lema II.5:}

$$
\begin{aligned}
& \Delta_{\mathcal{S}_{\xi \cup X}}\left(\mathcal{S}_{Y}\right)=\left(\bar{\varphi}^{-1} * D_{\mathcal{S}_{\xi \cup X}} \bar{\varphi}\right)\left(\mathcal{S}_{Y}\right)=\sum_{\substack{Y_{1} \cup Y_{2}=Y \\
Y_{1} \cap Y_{2}=\emptyset}} \bar{\varphi}^{-1}\left(\mathcal{S}_{Y_{1}}\right) \bar{\varphi}\left(\mathcal{S}_{\xi \cup X \cup Y_{2}}\right) \\
& =\sum_{\substack{Y_{1} \cup Y_{2}=Y \\
Y_{2} \cap(\xi \cup X)=\emptyset}}^{*} \bar{\varphi}^{-1}\left(\mathcal{S}_{Y_{1}}\right) e^{-U\left(\mathcal{S}_{\xi \cup X \cup Y_{2}}\right)-\beta_{0}\left(\left|\mathcal{S}_{\xi}\right|+\left|\mathcal{S}_{X}\right|+\left|\mathcal{S}_{Y_{2}}\right|\right)} \\
& =e^{-\beta_{0}\left|\mathcal{S}_{\xi}\right|} \sum_{\substack{Y_{1} \cup Y_{2}=Y \\
Y_{2} \cap(\xi \cup X)=\emptyset}}^{*} \bar{\varphi}^{-1}\left(\mathcal{S}_{Y_{1}}\right) e^{-\beta_{0}\left(\left|\mathcal{S}_{X}\right|+\left|\mathcal{S}_{Y_{2}}\right|\right)} e^{-U\left(\mathcal{S}_{X} \cup \mathcal{S}_{Y_{2}}\right)-U_{1}\left(\mathcal{S}_{X} \cup \mathcal{S}_{\xi}\right)-I_{1}\left(\mathcal{S}_{X} \cup \xi, \mathcal{S}_{Y_{2}}\right)} \\
& =e^{-\beta_{0}\left|\mathcal{S}_{\xi}\right|-U_{1}\left(\mathcal{S}_{X \cup \xi}\right)} \sum_{\substack{Y_{1} \cup Y_{2}=Y \\
Y_{2} \cap(\xi \cup X)=\emptyset}}^{*} \bar{\varphi}^{-1}\left(\mathcal{S}_{Y_{1}}\right) \varphi\left(\mathcal{S}_{X \cup Y_{2}}\right) e^{-I_{1}\left(\mathcal{S}_{X \cup \xi}, \mathcal{S}_{Y_{2}}\right)} \\
& =e^{-\beta_{0}\left|\mathcal{S}_{\xi}\right|-U_{1}\left(\mathcal{S}_{X \cup \xi}\right)} \sum_{\substack{Y_{1} \cup Y_{2}=Y \\
Y_{2} \cap(\xi \cup X)=\emptyset}}^{*} \bar{\varphi}^{-1}\left(\mathcal{S}_{Y_{1}}\right) \varphi\left(\mathcal{S}_{X \cup Y_{2}}\right) \sum_{T \subset Y_{2}} K_{1}\left(\mathcal{S}_{X \cup \xi}, \mathcal{S}_{T}\right) \\
& =e^{-\beta_{0}\left|\mathcal{S}_{\xi}\right|-U_{1}\left(\mathcal{S}_{X \cup \xi}\right)} \sum_{\substack{T \subset Y \\
T \cap(\xi \cup X)=\emptyset}} K_{1}\left(\mathcal{S}_{X^{\prime} \cup \xi}, \mathcal{S}_{T}\right) \sum_{\substack{Y_{1} \cup Y_{2}=Y^{\prime} \backslash T \\
Y_{2} \cap(\xi \cup X \cup T)=\emptyset}} \bar{\varphi}^{-1}\left(\mathcal{S}_{Y_{1}}\right) \varphi\left(\mathcal{S}_{X \cup T \cup Y_{2}}\right) \\
& =e^{-\beta_{0}\left|\mathcal{S}_{\xi}\right|-U_{1}\left(\mathcal{S}_{X \cup \xi}\right)} \sum_{\substack{T \subset Y \\
T \cap(\xi \cup X)=\emptyset}} K_{1}\left(\mathcal{S}_{X \cup \xi}, \mathcal{S}_{T}\right) \sum_{\substack{P \subset Y^{\prime} \backslash T \\
P \cap \xi \neq \emptyset}}(-1)^{N(P)} \Delta_{\mathcal{S}_{X \cup T \cup P}}\left(\mathcal{S}_{Y^{\prime} \backslash(T \cup P)}\right) .
\end{aligned}
$$

onde $\sum^{*}$ significa que a soma é feita somente sobre conjuntos de aglomerados sem sobreposição.

\section{Prova do lema II.6:}

Usando a estimativa obtida em (II.34) e o fato que existe no máximo $3^{\left|\mathcal{S}_{\xi}\right|+|\xi|} \leq 3^{2\left|\mathcal{S}_{\xi}\right|}$ diferentes formas sobre um aglomerado $\xi$ com mesmo comprimento $\left|\mathcal{S}_{\xi}\right|$ temos que, para $\beta_{0}$ suficientemente grande: 


$$
\begin{aligned}
\left.\frac{1}{N} \sum_{\substack{X \subset[0, N] \\
X \ni p}} \sum_{\substack{\mathcal{S}_{X} \\
\mid \varphi^{2}}} \mid \mathcal{S}_{X}\right)\left|\leq \frac{1}{N} \sum_{\substack{\xi \subset[0, N] \\
\xi \ni p}} \sum_{\mathcal{S}_{\xi}} \sum_{X, \mathcal{S}_{X}}\right| \bar{\varphi}^{T}\left(\mathcal{S}_{\xi \cup X}\right) \mid \leq \frac{1}{N} \sum_{\substack{\xi \subset[0, N] \\
\xi \ni p}} \sum_{\mathcal{S}_{\xi}} \frac{e^{-\frac{\beta_{0}}{2}\left(\left|\mathcal{S}_{\xi}\right|+1\right)}}{1-\kappa\left(\beta, \beta_{0}\right)} \\
\leq \frac{1}{N} \frac{e^{-\frac{\beta_{0}}{2}}}{1-\kappa\left(\beta, \beta_{0}\right)} \sum_{|\xi|=0}^{N+1} \sum_{l=|\xi|+1}\left(3^{2} e^{-\frac{\beta_{0}}{2}}\right)^{l} \\
=\frac{1}{N} \frac{9 e^{-\beta_{0}}}{\left(1-\kappa\left(\beta, \beta_{0}\right)\right)\left(1-9 e^{-\frac{\beta_{0}}{2}}\right)^{2}}\left[1-\left(9 e^{-\frac{\beta_{0}}{2}}\right)^{N+2}\right] \stackrel{N \rightarrow \infty}{\longrightarrow} 0 .
\end{aligned}
$$

Prova da Proposição II.2: Os aglomerados são subconjuntos de $\mathbb{Z}$, logo usando a notação introduzida na seção II.6 podemos representar o intervalo $[0, N] \subset \mathbb{Z}$ por $[0, N]=\bigcup_{j=1}^{k+1}\left[a_{j-1}+1, a_{j}\right] \bigcup\{0\}$.

Se $X \subset[0, N]$ é um conjunto de aglomerados, podemos particioná-lo em subconjuntos de aglomerados $Y_{i}, i=1, \ldots, \nu$ tais que, para cada $Y_{i}$ e um intervalo $\left[a_{j-1}+1, a_{j}\right]$ podem ocorrer:

(i) $Y_{i} \cap\left[a_{j-1}+1, a_{j}\right]=\emptyset$,

(ii) $Y_{i} \subset\left[a_{j-1}+1, a_{j}\right]$ ou

(iii) $Y_{i} \not \subset\left[a_{j-1}+1, a_{j}\right]$ e $Y_{i} \cap\left[a_{j-1}+1, a_{j}\right] \neq \emptyset$.

Para o primeiro caso temos que $\sum_{\xi \in Y_{i}} g_{j}\left(\mathcal{S}_{\xi}\right)=0$, portanto $f_{N}\left(\mathcal{S}_{Y_{i}}\right)=0$.

Para o caso (ii), $f_{N}\left(\mathcal{S}_{Y_{i}}\right)=s_{j} \sum_{\xi \in Y_{i}} g_{j}\left(\mathcal{S}_{\xi}\right) \delta \mathcal{S}_{\xi}=s_{j} \delta \mathcal{S}_{Y_{i}^{\prime}}$.

A situação (iii) implica que $Y_{i} \cap\left\{\left[a_{j-1}+1, a_{j}\right] \cup A_{j}\right\} \neq \emptyset$, onde $A_{j}=\left\{a_{j-1}, a_{j}+1\right\}$. Quando $Y_{i} \cap\{0\} \neq \emptyset$ temos que $Y_{i} \cap A_{1} \neq \emptyset$ e, portanto está contido no caso (iii) para $j=1$. Vamos representar os conjuntos de aglomerados que se encontram no caso (iii) por $Y^{*}$. 
A partir dos fatos acima obtemos que

$$
\begin{aligned}
& \sum_{X \subset[0, N]} \sum_{\mathcal{S}_{X}} \bar{\varphi}^{T}\left(\mathcal{S}_{X}\right) e^{-\left(\beta-\beta_{0}\right)\left|\mathcal{S}_{X}\right|}\left(e^{i \frac{f_{N}\left(\mathcal{S}_{X}\right)}{\sigma \sqrt{N}}}-1\right) \\
&=\sum_{j=1}^{k+1} \sum_{Y \subset\left[a_{j-1}=1, a_{j}\right]} \sum_{\mathcal{S}_{Y}} \bar{\varphi}^{T}\left(\mathcal{S}_{Y^{\prime}}\right) e^{-\left(\beta-\beta_{0}\right)\left|\mathcal{S}_{Y}\right|}\left(e^{i \frac{s_{j} \delta S_{Y}}{\sigma \sqrt{N}}}-1\right) \\
&+\sum_{j=1}^{k+1} \sum_{Y^{*} \cap A_{j} \neq \emptyset} \sum_{\mathcal{S}_{Y^{*}}} \bar{\varphi}^{T}\left(\mathcal{S}_{Y^{*}}\right) e^{-\left(\beta-\beta_{0}\right)\left|\mathcal{S}_{Y^{*}}\right|}\left(e^{i \frac{f_{N}\left(\mathcal{S}_{Y^{*}}\right)}{\sigma \sqrt{N}}}-1\right)
\end{aligned}
$$

A contribuição do segundo termo à direita de (B.5) é dada por:

$$
\begin{aligned}
\left|\sum_{j=1}^{k+1} \sum_{Y^{*} \cap A_{j} \neq \emptyset} \sum_{\mathcal{S}_{Y^{*}}} \bar{\varphi}^{T}\left(\mathcal{S}_{Y^{*}}\right) e^{-\left(\beta-\beta_{0}\right)\left|\mathcal{S}_{Y^{\prime}}\right|}\left(e^{i \frac{f_{N}\left(S_{Y^{*}}\right)}{\sigma \sqrt{N}}}-1\right)\right| \\
\leq 2 \sum_{j=1}^{k+1} \sum_{Y^{*} \cap A_{j} \neq \emptyset \emptyset \mathcal{S}_{Y^{*}}}\left|\bar{\varphi}^{T}\left(\mathcal{S}_{Y^{*}}\right)\right| \leq 2(k+1) \sup _{p \in A^{*}} \sum_{\substack{Y \subset[0, N] \\
Y_{\ni p}}} \sum_{\mathcal{S}_{Y}}\left|\bar{\varphi}^{T}\left(\mathcal{S}_{Y^{\prime}}\right)\right| \\
\quad=o(N) \quad \text { quando } N \rightarrow \infty .
\end{aligned}
$$

pelo lema II.6 e, onde $A^{*}=\bigcup_{j=1}^{k+1} A_{j}$. 


\section{Referências Bibliográficas}

[Bil] Billingsley, P. (1968). Weak Convergence of Probability Measures, New York: John Wiley.

[Bry] Brydges, D.C. (1984). A short course on cluster expansions, Les Houches, Session XLIII - Phénomènes critiques, systèmes aléatoires, théories de jauge - K. Osterwalder and R. Stora, eds., 129-183.

[Gal] Gallavotti, G. (1972). The Phase Separation Line in the Two-Dimensional Ising Model, Commun. Math. Phys., 27, 103-136.

[GMM] Gallavotti, G.;Martin-Löf, A. and Miracle-Solé, S. (1971). Some Problems Connected with the Description of Coexisting Phases at Low Temperatures in the Ising Model, Lecture Notes in Physics, 20, 162-204.

[GMa] Gallavotti, G. and Martin-Löf, A. (1972). Surface Tension in the Ising Model, Commun. Math. Phys., 25, 87-126.

[GMi] Gallavotti, G. and Miracle-Solé, S. (1968). Correlation Functions of a Lattice System, Commun. Math. Phys., 7, 274-288.

[GK] Gnedenko, B.V. and Kolmogorov, A.N. (1954). Limit distribuitions for sums of independent random variables, Cambridge: Addison-Wesley.

[Hig] Higuchi, Y. (1979). On some Limit Theorems Related to the Phase Separation Line in the Two-Dimensional Ising Model, Z.W.verw.Gebiete, 50, 287-315.

[Jam] James, B.R. (1981). Probabilidade: um curso em nivel intermediário, Rio de Janeiro: IMPA-CNPq. 
[KS] Kindermann, R. and Snell, J.L. (1980). Markov random fields and their applications, A.M.S., Series: Contemporary Mathematics, vol 1, Providence, Rhode Island.

[MS] Minlos, R.A. and Sină, Ja.G. (1968). The phenomenon of "phase separation" at low temperatures in some lattice models of a gas II, Trans. Moscow Math. Soc., 19, 121-196.

[Lig] Ligget, T. (1968). An invariance principle for conditional sums of independent random variables, J.Math.Mech., 18, 559-570.

[Pet] Petrov, V.V. (1975). Sums of Independent Random Variables, Springer-Verlag.

[Rue] Ruelle, D. (1969). Statistical Mechanics, New York: Benjamin. 